Supporting Information for:

\title{
Phosphine-Catalyzed Synthesis of Highly Functionalized Coumarins
}

\author{
Christopher E. Henry, and Ohyun Kwon* \\ Department of Chemistry and Biochemistry, University of California, Los Angeles, California \\ 90095-1569, USA
}

General Information: All reactions were performed under an argon atmosphere using dry solvents and anhydrous conditions, unless otherwise noted. THF and diethyl ether were distilled over sodium/benzophenone ketyl; dichloromethane and benzene were distilled from $\mathrm{CaH}_{2}$. Reactions were monitored through thin-layer chromatography (TLC) on $0.25 \mathrm{~mm}$ E. Merck silica gel plates $(60 \mathrm{~F}-254)$ and visualized under UV light and/or anisaldehyde or permanganate staining. Flash column chromatography was performed using E. Merck silica gel 60 (230-400 mesh) and compressed air. IR spectra were recorded on a Perkin-Elmer pargon 1600 FT-IR spectrometer. NMR spectra were obtained on a Bruker Avance-500, ARX-500 or ARX-400 instrument, as indicated, and calibrated using residual chloroform as an internal reference (7.26 ppm for ${ }^{1} \mathrm{H}$ NMR; $77.00 \mathrm{ppm}$ for ${ }^{13} \mathrm{C}$ NMR). Data for ${ }^{1} \mathrm{H}$ NMR spectra are reported as follows: chemical shift ( $\delta \mathrm{ppm})$, multiplicity, coupling constant $(\mathrm{Hz})$, and integration. Data for ${ }^{13} \mathrm{C} N M R$ spectra are reported in terms of chemical shift and multiplicities, with coupling constants $(\mathrm{Hz})$ in the case of $J_{\mathrm{CF}}$ coupling. The following abbreviations are used to explain the multiplicities: $\mathrm{s}=$ singlet; $\mathrm{d}=$ doublet; $\mathrm{t}=$ triplet; $\mathrm{q}=$ quartet; $\mathrm{m}=$ multiplet; $\mathrm{br}=$ broad; app = apparent. Highresolution matrix-assisted laser desorption/ionization (MALDI) mass spectra were recorded using a dihydroxybenzoic acid (DHB) matrix and an Applied Biosystems Voyager-DE STR operated in reflector mode with external calibration at an accelerating voltage of 20,000 V. Highresolution EI mass spectra were recorded after rapid thermal vaporization of samples deposited on a desorption/ionization filament that was directly inserted into the electron ionization (EI, 70 $\mathrm{eV}, 200{ }^{\circ} \mathrm{C}$ ) source of a triple-sector high-resolution instrument (VG/Micromass Autospec) tuned to 8,000 static resolution (M/DM, 10\% valley), using perfluorinated kerosene (formula weight: 705; Lancaster Synthesis, Inc., NH) as the internal calibrant. High-resolution chemical ionization (CI) mass spectra were obtained using a Micromass GCT Gas Chromatograph EI/CI TOF instrument. Atmospheric pressure chemical ionization (APCI) was performed using a SCIEX II instrument equipped with an optional Applied Biosystems/MSD Sciex QSTAR-XL hybrid Quad- 
TOF.

\section{Synthesis of $o$-Hydroxycinnamate Derivatives}

$o$-Hydroxycinnamate derivatives were prepared using a modified procedure reported by Bunce and Schilling. ${ }^{1}$ As an example, the synthesis of 3-(5-fluoro-2-hydroxyphenyl)acrylic acid ethyl ester $\mathbf{S 1}$ is provided.
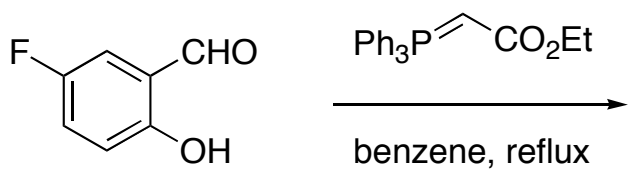<smiles>CCOC(=O)/C=C/c1cc(F)ccc1O</smiles>

S1

5-Fluorosalicylaldehyde (7.1 mmol, $1.0 \mathrm{~g})$ and (carbethoxymethylene)triphenylphosphorane (14.3 mmol, $4.97 \mathrm{~g})$ were dissolved in benzene $(30 \mathrm{~mL})$. The resultant solution was heated under reflux overnight (ca. $15 \mathrm{~h}$ ). Two-thirds of the solvent was removed through rotary evaporation and the crude residue was loaded directly onto a silica gel column and eluted with hexane/ethyl acetate (4:1) to yield $\mathbf{S 1}\left(1.378 \mathrm{~g}, 6.55 \mathrm{mmol}, 92 \%\right.$ yield) as a pale yellow solid: m.p. $81-84{ }^{\circ} \mathrm{C}$; IR (film) $v_{\max } 3393,2986,1686,1629,1506,1443,1188 \mathrm{~cm}^{-1} ;{ }^{1} \mathrm{H}$ NMR $\left(400 \mathrm{MHz}, \mathrm{CDCl}_{3}\right) \delta$ $7.97(\mathrm{~d}, J=16.1 \mathrm{~Hz}, 1 \mathrm{H}), 7.17(\mathrm{dd}, J=9.1,3.0 \mathrm{~Hz}, 1 \mathrm{H}), 6.95(\mathrm{td}, J=8.5,3.0 \mathrm{~Hz}, 1 \mathrm{H}), 6.79(\mathrm{dd}$, $J=8.8,4.5 \mathrm{~Hz}, 1 \mathrm{H}), 6.58(\mathrm{~d}, J=16.1 \mathrm{~Hz}, 1 \mathrm{H}), 6.23(\mathrm{br} \mathrm{s}, 1 \mathrm{H}), 4.28(\mathrm{q}, J=7.1 \mathrm{~Hz}, 2 \mathrm{H}), 1.34(\mathrm{t}$, $J=7.1 \mathrm{~Hz}, 3 \mathrm{H}) ;{ }^{13} \mathrm{C} \mathrm{NMR}\left(100 \mathrm{MHz}, \mathrm{CDCl}_{3}\right) \delta 167.7,156.0,151.1,139.0,122.8,119.7,117.9$ $\left(J_{\mathrm{CF}}=23.2 \mathrm{~Hz},\right), 117.3\left(J_{\mathrm{CF}}=7.9 \mathrm{~Hz}\right), 114.4\left(J_{\mathrm{CF}}=23.3 \mathrm{~Hz}\right), 60.8,14.3$; HRMS (EI) calcd for $\mathrm{C}_{11} \mathrm{H}_{11} \mathrm{FO}_{3}\left[\mathrm{M}^{+}\right] 210.0692$, found 210.0684 .

The following o-hydroxycinnamates-ethyl 2-hydroxy cinnamate, ${ }^{1}$ ethyl 5-bromo-2hydroxycinnamate, ${ }^{2}$ ethyl 5-nitro-2-hydroxycinnamate, ${ }^{2}$ ethyl 3-methyl-2-hydroxycinnamate, ${ }^{3}$ ethyl 3-methoxy-2-hydroxycinnamate, ${ }^{2}$ ethyl 4-methoxy-2-hydroxycinnamate, ${ }^{1}$ and ethyl 5methoxy-2-hydroxycinnamate ${ }^{3}$ - have been synthesized previously; their spectral data are reported in the references indicated.

\section{Attempted Allenoate Syntheses}

Efforts to directly couple allenoic acid or 3-butynoic acid, synthesized through Jones oxidation of 3-butyn-1-ol, to the salicylaldehyde or cinnamate derivatives were unsuccessful. Coupling 
reactions utilizing DCC, ${ }^{4}$ PyBroP, ${ }^{5}$ HATU, ${ }^{6} \mathrm{HBTU}^{7}{ }^{7} \mathrm{TCT}^{8}{ }^{8} \mathrm{Hf}(\mathrm{IV})$ salts, ${ }^{9}$ and Furukawa's procedure $^{10}$ were all attempted. Complications in these coupling reactions were anticipated because of the susceptibility of the product allenoates to undergo nucleophilic attack by either a base or a byproduct of the coupling reactions. As an example, the attempted coupling of ethyl $o$ hydroxycinnamate and butynoic acid with HBTU is described.

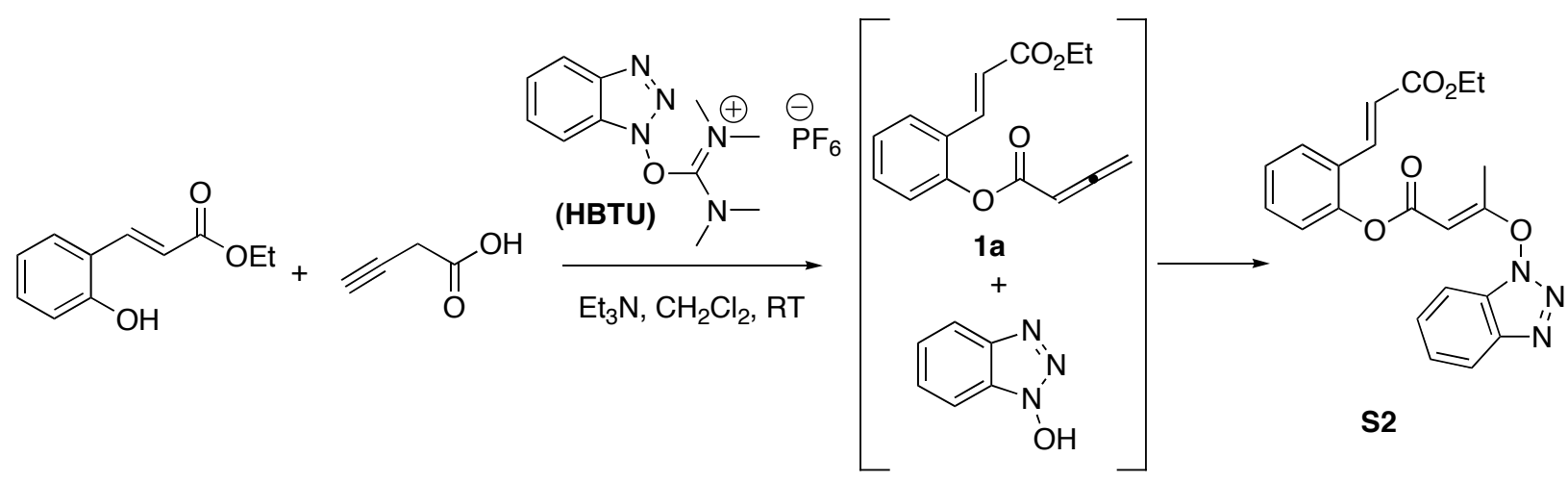

A solution of 3-butynoic acid $(0.254 \mathrm{~g}, 3.0 \mathrm{mmol})$ and $\mathrm{Et}_{3} \mathrm{~N}(418 \mu \mathrm{L}, 3.0 \mathrm{mmol})$ in $\mathrm{CH}_{2} \mathrm{Cl}_{2}(10$ $\mathrm{mL})$ was added to a stirred solution of $o$-hydroxy cinnamate $(0.297 \mathrm{~g}, 1.52 \mathrm{mmol})$ and HBTU (0.569 g, $1.5 \mathrm{mmol})$ in $\mathrm{CH}_{2} \mathrm{Cl}_{2}(10 \mathrm{~mL})$. The mixture was stirred at room temperature and monitored using TLC. The desired allenoate 1a was produced as an intermediate (TLC, cospotted with a sample of 1a), but was quickly consumed. The allenoate $1 \mathbf{a}\left\{R_{\mathrm{f}}\right.$ [hexane/ethyl acetate $(2: 1)]=0.41$; anisaldehyde stain: bright red $\}$ reached its maximum intensity ca. 5 min. into the reaction before a large amount of ethyl 2-hydroxycinnamate $\left\{R_{\mathrm{f}}\right.$ [hexanes/ethyl acetate $(2: 1)]=0.30$; fluorescent $\}$ was consumed. After $2.5 \mathrm{~h}$, ethyl 2-hydroxycinnamate was completely consumed. The reaction mixture was quenched with $\mathrm{H}_{2} \mathrm{O}(20 \mathrm{~mL})$ and extracted with EtOAc $(2 \times 20 \mathrm{~mL})$. The combined organic fractions were concentrated and chromatographed (hexane/ethyl acetate, 4:1) to yield S2 (440 mg, 74\%) as a clear oil; IR (film) $v_{\max } 1741,1713$, 1657, 1318, $1281 \mathrm{~cm}^{-1} ;{ }^{1} \mathrm{H}$ NMR $\left(500 \mathrm{MHz}, \mathrm{CDCl}_{3}\right) \delta 8.06(\mathrm{~d}, J=8.4 \mathrm{~Hz}, 1 \mathrm{H}), 7.59-7.52(\mathrm{~m}$, 4H), $7.43(\mathrm{td}, J=8.1,1.1 \mathrm{~Hz}, 1 \mathrm{H}), 7.29(\mathrm{td}, J=6.9,1.2 \mathrm{~Hz}, 1 \mathrm{H}), 7.16(\mathrm{t}, J=7.4 \mathrm{~Hz}, 1 \mathrm{H}), 6.98$ $(\mathrm{d}, J=8.1 \mathrm{~Hz}, 1 \mathrm{H}), 6.32(\mathrm{~d}, J=16.1 \mathrm{~Hz}, 1 \mathrm{H}), 5.15(\mathrm{~s}, 1 \mathrm{H}), 4.17(\mathrm{q}, J=7.1 \mathrm{~Hz}, 2 \mathrm{H}), 2.61(\mathrm{~s}, 3 \mathrm{H})$, $1.25(\mathrm{t}, J=7.1 \mathrm{~Hz}, 3 \mathrm{H}) ;{ }^{13} \mathrm{C} \mathrm{NMR}\left(125 \mathrm{MHz}, \mathrm{CDCl}_{3}\right) \delta 173.2,166.2,163.9,148.7,143.1,137.4$, $130.8,129.2,127.5,127.3,127.0,126.2,125.2,122.8,120.5,120.2,108.2,95.4,60.3,15.5$, 14.0; MS (EI) calcd for $\mathrm{C}_{21} \mathrm{H}_{19} \mathrm{~N}_{3} \mathrm{O}_{5}\left[\mathrm{M}^{+}\right]$393.13, found 393.13. 


\section{Allenoate Synthesis}

The desired allenoates were synthesized using Mukaiyama coupling reagents. ${ }^{11}$ The coupling reagent byproduct, pyridin-2-one, did not react with the product allenoates under the reaction conditions. As a typical example of the experimental procedure used for the synthesis of 2-[(2ethoxycarbonyl)vinyl]phenyl allenoates, the synthesis of 2-[(2-ethoxycarbonyl)vinyl]phenyl allenoate $1 \mathbf{a}$ is described.
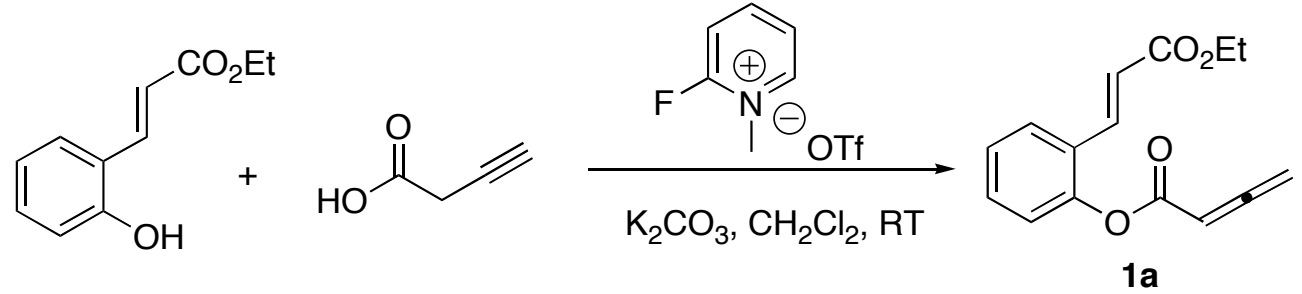

Ethyl $o$-hydroxycinnamate (192 mg, $1.00 \mathrm{mmol}$ ), 3-butynoic acid (96 mg, $1.1 \mathrm{mmol})$, 2-fluoro-1methylpyridiumium triflate $(287 \mathrm{mg}, 1.1 \mathrm{mmol}){ }^{12}$ and potassium carbonate $(304 \mathrm{mg}, 2.2 \mathrm{mmol})$ were weighed in a 50-mL round-bottom flask and then distilled $\mathrm{CH}_{2} \mathrm{Cl}_{2}(10 \mathrm{~mL})$ was added to the mixture. Upon stirring, the faint-yellow suspension became a clear solution over the course of an hour. The reaction was monitored using TLC (hexane/ethyl acetate, 2:1) and visualized through anisaldehyde staining (the product aryl allenoates stain bright red). After $15 \mathrm{~h}$, the reaction was complete and a large amount of yellow precipitate was observed. To remove the precipitated salts and pyridin-2-one, the crude reaction mixture was filtered through a small pad of silica with $\mathrm{CH}_{2} \mathrm{Cl}_{2}$ before concentration by rotary evaporation. The crude residue was purified by column chromatography (hexane/ethyl acetate, 4:1) to yield 1a (185 $\mathrm{mg}, 72 \%$ yield) as a clear oil; IR (neat) $v_{\max } 3068,2980,2871,1968,1940,1738,1718,1638 \mathrm{~cm}^{-1} ;{ }^{1} \mathrm{H}$ NMR (500 MHz, $\left.\mathrm{CDCl}_{3}\right) \delta 7.78(\mathrm{~d}, J=16.1 \mathrm{~Hz}, 1 \mathrm{H}), 7.62(\mathrm{dd}, J=7.8,1.5 \mathrm{~Hz}, 1 \mathrm{H}) 7.40(\mathrm{td}, J=7.8,1.4 \mathrm{~Hz}, 1 \mathrm{H})$, $7.26(\mathrm{td}, J=6.6,0.6 \mathrm{~Hz}, 1 \mathrm{H}), 7.20(\mathrm{dd}, J=8.2,0.9 \mathrm{~Hz}, 1 \mathrm{H}), 6.48(\mathrm{~d}, J=16.1 \mathrm{~Hz}, 1 \mathrm{H}), 5.86(\mathrm{t}, J$ $=6.5 \mathrm{~Hz}, 1 \mathrm{H}), 5.41(\mathrm{~d}, J=6.5 \mathrm{~Hz}, 2 \mathrm{H}), 4.25(\mathrm{q}, J=7.1 \mathrm{~Hz}, 2 \mathrm{H}), 1.33(\mathrm{t}, J=7.1 \mathrm{~Hz}, 3 \mathrm{H}) ;{ }^{13} \mathrm{C}$ NMR $\left(125 \mathrm{MHz}, \mathrm{CDCl}_{3}\right) \delta 217.0,166.7,163.7,149.3,138.0,131.0,127.9,127.2,126.3,123.1$, 120.5, 87.4, 80.1, 60.6, 14.3; HRMS (MALDI) calcd for $\mathrm{C}_{15} \mathrm{H}_{14} \mathrm{O}_{4} \mathrm{Na}\left[(\mathrm{M}+\mathrm{Na})^{+}\right] 281.0784$, found 281.0785.

Spectral data for all additional allenoates are provided below. 


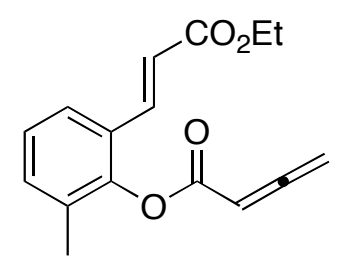

$1 b$

Allenoate 1b. 64\% Yield; clear oil; IR (neat) $\nu_{\max }$ 3060, 1968, 1940, 1740, 1718, $1637 \mathrm{~cm}^{-1} ;{ }^{1} \mathrm{H}$ NMR (400 MHz, $\left.\mathrm{CDCl}_{3}\right) \delta 7.72(\mathrm{~d}, J=16.1 \mathrm{~Hz}, 1 \mathrm{H}), 7.46(\mathrm{dm}, J=7.5 \mathrm{~Hz}, 1 \mathrm{H}), 7.26(\mathrm{dm}, J=$ $6.4,1 \mathrm{H}), 7.14(\mathrm{t}, J=7.6 \mathrm{~Hz}, 1 \mathrm{H}), 6.44(\mathrm{~d}, J=16.1 \mathrm{~Hz}, 1 \mathrm{H}), 5.88(\mathrm{t}, J=6.5 \mathrm{~Hz}, 1 \mathrm{H}), 5.40(\mathrm{~d}, J=$ $6.47 \mathrm{~Hz}, 2 \mathrm{H}), 4.25$ (q, $J=7.1 \mathrm{~Hz}, 2 \mathrm{H}), 2.20(\mathrm{~s}, 3 \mathrm{H}), 1.33(\mathrm{t}, J=7.1 \mathrm{~Hz}, 3 \mathrm{H}) ;{ }^{13} \mathrm{C}$ NMR $(100$ $\left.\mathrm{MHz}, \mathrm{CDCl}_{3}\right) \delta 217.1,166.8,163.4,148.2,138.4,132.8,131.6,129.1,126.3,125.4,120.5,87.0$, 80.0, 60.5, 16.3, 14.3; HRMS (MALDI) calcd for $\mathrm{C}_{16} \mathrm{H}_{17} \mathrm{O}_{4}\left[(\mathrm{M}+\mathrm{H})^{+}\right] 273.1121$, found 273.1123 .

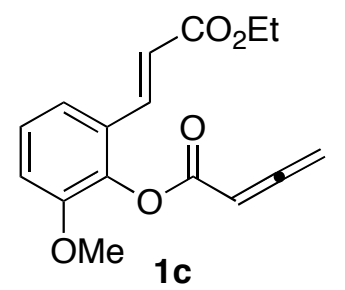

Allenoate 1c. 77\% Yield; clear oil; IR (neat) $v_{\max } 2984,1964,1940,1740,1724 \mathrm{~cm}^{-1}$; ${ }^{1} \mathrm{H}$ NMR $\left(500 \mathrm{MHz}, \mathrm{CDCl}_{3}\right) \delta 7.74(\mathrm{~d}, J=16.1 \mathrm{~Hz}, 1 \mathrm{H}), 7.21-7.20(\mathrm{~m}, 2 \mathrm{H}), 6.84-6.83(\mathrm{~m}, 1 \mathrm{H}), 6.45(\mathrm{~d}, J$ $=16.1 \mathrm{~Hz}, 1 \mathrm{H}), 5.89(\mathrm{t}, J=6.5 \mathrm{~Hz}, 1 \mathrm{H}), 5.37(\mathrm{~d}, J=6.5 \mathrm{~Hz}, 2 \mathrm{H}), 4.25(\mathrm{q}, J=7.1 \mathrm{~Hz}, 2 \mathrm{H}), 3.82$ $(\mathrm{s}, 3 \mathrm{H}), 1.31(\mathrm{t}, J=7.1 \mathrm{~Hz}, 3 \mathrm{H}) ;{ }^{13} \mathrm{C} \mathrm{NMR}\left(125 \mathrm{MHz}, \mathrm{CDCl}_{3}\right) \delta 217.0,166.6,163.0,151.7$, $138.7,137.9,128.4,126.6,120.9,119.0,113.6,87.0,80.0,60.5,56.1,14.2$; HRMS (MALDI) calcd for $\mathrm{C}_{16} \mathrm{H}_{16} \mathrm{O}_{5} \mathrm{Na}\left[(\mathrm{M}+\mathrm{Na})^{+}\right] 311.0890$, found 311.0881 .

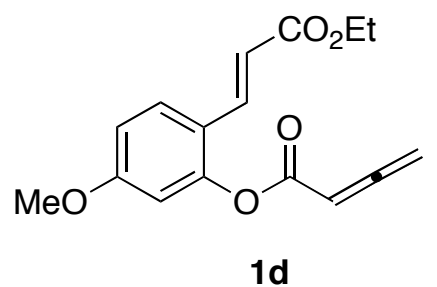

Allenoate 1d. 71\% Yield; white solid; m.p. 56-58 ${ }^{\circ} \mathrm{C}$; IR (neat) $v_{\max }$ 3069, 2986, 1967, 1937, 1740, 1709, 1633, 1611, 1504, 1299, $1263 \mathrm{~cm}^{-1} ;{ }^{1} \mathrm{H}$ NMR $\left(500 \mathrm{MHz}, \mathrm{CDCl}_{3}\right) \delta 7.69$ (d, $J=16.1$ 
$\mathrm{Hz}, 1 \mathrm{H}), 7.53(\mathrm{~d}, J=8.8 \mathrm{~Hz}, 1 \mathrm{H}), 6.79(\mathrm{dd}, J=11.3,2.6 \mathrm{~Hz}, 1 \mathrm{H}), 6.72(\mathrm{~d}, J=2.5 \mathrm{~Hz}, 1 \mathrm{H}), 6.35$ $(\mathrm{d}, J=16.0 \mathrm{~Hz}, 1 \mathrm{H}), 5.84(\mathrm{t}, J=6.5 \mathrm{~Hz}, 1 \mathrm{H}), 5.39(\mathrm{~d}, J=6.5 \mathrm{~Hz}, 2 \mathrm{H}), 4.22(\mathrm{q}, J=7.1 \mathrm{~Hz}, 2 \mathrm{H})$, 3.79 (s, 3H), 1.30 (t, J=7.1 Hz, 3H); $\left.{ }^{13} \mathrm{C} \mathrm{NMR} \mathrm{(125} \mathrm{MHz,} \mathrm{CDCl}_{3}\right) \delta$ 216.9, 167.0, 163.4, 161.7, 150.4, 137.7, 128.7, 119.6, 117.7, 112.8, 108.2, 87.2, 80.0, 60.2, 55.5, 14.2; HRMS (EI) calcd for $\mathrm{C}_{16} \mathrm{H}_{16} \mathrm{O}_{5}\left[\mathrm{M}^{+}\right] 288.0998$, found 288.1003.

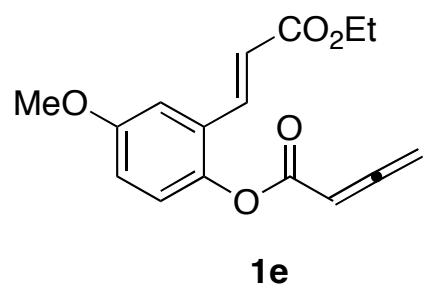

Allenoate 1e. 73\% Yield; clear oil; IR (neat) $v_{\max }$ 3069, 2987, 1968, 1938, 1736, 1712, 1637 , 1494, $1184 \mathrm{~cm}^{-1} ;{ }^{1} \mathrm{H}$ NMR (500 MHz, $\left.\mathrm{CDCl}_{3}\right) \delta 7.71(\mathrm{~d}, J=16.1 \mathrm{~Hz}, 1 \mathrm{H}), 7.10-7.07$ (m, 2H), $6.93(\mathrm{dd}, J=8.9,3.0 \mathrm{~Hz}, 1 \mathrm{H}), 6.43(\mathrm{~d}, J=16.1 \mathrm{~Hz}, 1 \mathrm{H}), 5.84(\mathrm{t}, J=6.5 \mathrm{~Hz}, 1 \mathrm{H}), 5.38(\mathrm{~d}, J=6.5$ $\mathrm{Hz}, 2 \mathrm{H}), 4.24$ (q, $J=7.1 \mathrm{~Hz}, 2 \mathrm{H}), 3.80(\mathrm{~s}, 3 \mathrm{H}), 1.32$ (t, $J=7.1 \mathrm{~Hz}, 3 \mathrm{H}) ;{ }^{13} \mathrm{C} \mathrm{NMR}(125 \mathrm{MHz}$, $\left.\mathrm{CDCl}_{3}\right) \delta 216.9,166.6,164.0,157.3,143.0,138.0,127.7,123.9,120.5,116.8,111.8,87.3,79.9$, 60.5, 55.6, 14.2; HRMS (EI) calcd for $\mathrm{C}_{16} \mathrm{H}_{16} \mathrm{O}_{5}\left[\mathrm{M}^{+}\right]$288.0998, found 288.0991.

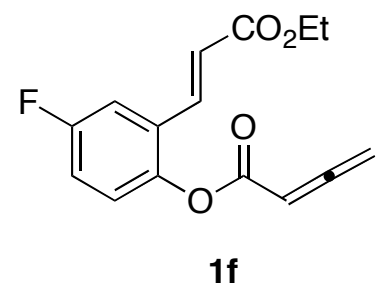

Allenoate 1f. $45 \%$ Yield; white solid; m.p. $54-55^{\circ} \mathrm{C}$; IR (film) $v_{\max } 3073,2989,1969,1937$, 1740, 1713, 1639, $1488 \mathrm{~cm}^{-1}$; ${ }^{1} \mathrm{H}$ NMR $\left(500 \mathrm{MHz}, \mathrm{CDCl}_{3}\right) \delta 7.69$ (d, $\left.J=16.1 \mathrm{~Hz}, 1 \mathrm{H}\right), 7.30$ (dd, $J=9.0,2.9 \mathrm{~Hz}, 1 \mathrm{H}), 7.17(\mathrm{dd}, J=9.0,4.9 \mathrm{~Hz}, 1 \mathrm{H}), 7.10(\mathrm{td}, J=7.6,2.9 \mathrm{~Hz}, 1 \mathrm{H}), 6.44(\mathrm{~d}, J=$ $16.1 \mathrm{~Hz}, 1 \mathrm{H}), 5.85$ (t, $J=6.4 \mathrm{~Hz}, 1 \mathrm{H}), 5.41(\mathrm{~d}, J=6.4 \mathrm{~Hz}, 2 \mathrm{H}), 4.26(\mathrm{q}, J=7.1 \mathrm{~Hz}, 2 \mathrm{H}), 1.32(\mathrm{t}$, $J=7.1,3 \mathrm{H}) ;{ }^{13} \mathrm{C}$ NMR $\left(125 \mathrm{MHz}, \mathrm{CDCl}_{3}\right) \delta 217.1,166.3,163.7,160.2\left(\mathrm{~d}, J_{\mathrm{CF}}=245.5 \mathrm{~Hz}\right)$, $145.2,136.9,128.8\left(\mathrm{~d}, J_{\mathrm{CF}}=8.6 \mathrm{~Hz}\right), 124.6\left(\mathrm{~d}, J_{\mathrm{CF}}=8.7 \mathrm{~Hz}\right), 121.6,117.7\left(\mathrm{~d}, J_{\mathrm{CF}}=23.6 \mathrm{~Hz}\right)$, $113.8\left(\mathrm{~d}, J_{\mathrm{CF}}=24.0 \mathrm{~Hz}\right), 87.2,80.2,60.7,14.3$; HRMS $(\mathrm{EI})$ calcd for $\mathrm{C}_{15} \mathrm{H}_{13} \mathrm{O}_{4} \mathrm{~F}\left[\mathrm{M}^{+}\right]$276.0798, found 276.0801 . 


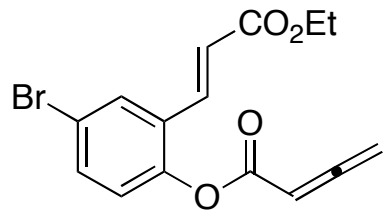

$1 \mathrm{~g}$

Allenoate 1g. 38\% Yield; white powder; m.p. $77-79{ }^{\circ} \mathrm{C}$; IR (film) $v_{\max } 2984,1961,1736,1718$, 1638, 1474, 1215, $1187 \mathrm{~cm}^{-1} ;{ }^{1} \mathrm{H}$ NMR $\left(500 \mathrm{MHz}, \mathrm{CDCl}_{3}\right) \delta 7.74(\mathrm{~d}, J=2.4 \mathrm{~Hz}, 1 \mathrm{H}), 7.68(\mathrm{~d}, J$ $=16.1 \mathrm{~Hz}, 1 \mathrm{H}), 7.50(\mathrm{dd}, J=8.7,2.4 \mathrm{~Hz}, 1 \mathrm{H}), 7.10(\mathrm{~d}, J=8.7 \mathrm{~Hz}, 1 \mathrm{H}), 6.47(\mathrm{~d}, J=16.1 \mathrm{~Hz}$, 1H), 5.85 (t, $J=6.5 \mathrm{~Hz}, 1 \mathrm{H}), 5.42(\mathrm{~d}, J=6.4 \mathrm{~Hz}, 2 \mathrm{H}), 4.26(\mathrm{q}, J=7.1 \mathrm{~Hz}, 2 \mathrm{H}), 1.33$ (t, $J=7.1$ $\mathrm{Hz}, 3 \mathrm{H}) ;{ }^{13} \mathrm{C} \mathrm{NMR}\left(125 \mathrm{MHz}, \mathrm{CDCl}_{3}\right) \delta 217.2,166.3,163.4,148.2,136.6,133.6,130.5,129.2$, 124.8, 121.8, 119.4, 87.2, 80.2, 60.7, 14.3; HRMS (EI) calcd for $\mathrm{C}_{15} \mathrm{H}_{13}{ }^{79} \mathrm{BrO}_{4}\left[\mathrm{M}^{+}\right] 335.9997$, found 335.9988; calcd for $\mathrm{C}_{15} \mathrm{H}_{13}{ }^{81} \mathrm{BrO}_{4}\left[\mathrm{M}^{+}\right]$337.9977, found 337.9992 .

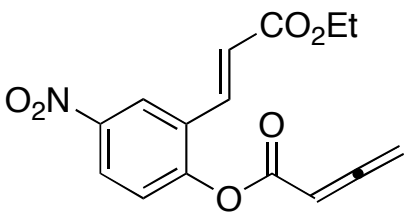

1h

Allenoate 1h. 45\% Yield; white soild; m.p. $104-106{ }^{\circ} \mathrm{C}$; IR (film) $v_{\max } 1968,1940,1736,1711$ 1648, $1462 \mathrm{~cm}^{-1} ;{ }^{1} \mathrm{H}$ NMR $\left(500 \mathrm{MHz}, \mathrm{CDCl}_{3}\right) \delta 8.51(\mathrm{~d}, J=2.6 \mathrm{~Hz}, 1 \mathrm{H}), 8.26(\mathrm{dd}, J=9.0,2.7$ $\mathrm{Hz}, 1 \mathrm{H}), 7.78$ (d, $J=16.1 \mathrm{~Hz}, 1 \mathrm{H}), 7.45$ (d, $J=9.0 \mathrm{~Hz}, 1 \mathrm{H}), 6.61(\mathrm{~d}, J=16.1 \mathrm{~Hz}, 1 \mathrm{H}), 5.88$ (t, $J$ $=6.4 \mathrm{~Hz}, 1 \mathrm{H}), 5.47(\mathrm{~d}, J=6.4 \mathrm{~Hz}, 2 \mathrm{H}), 4.29(\mathrm{q}, J=7.1 \mathrm{~Hz}, 2 \mathrm{H}), 1.35(\mathrm{t}, J=7.1 \mathrm{~Hz}, 3 \mathrm{H}) ;{ }^{13} \mathrm{C}$ NMR $\left(125 \mathrm{MHz}, \mathrm{CDCl}_{3}\right) \delta 217.5,166.0,162.7,153.4,145.5,135.8,128.6,125.4,124.1,123.3$, 123.2, 87.0, 80.6, 61.0, 14.2; HRMS (MALDI) calcd for $\mathrm{C}_{15} \mathrm{H}_{13} \mathrm{NO}_{6} \mathrm{Na}\left[(\mathrm{M}+\mathrm{Na})^{+}\right] 326.0635$, found 326.0633 .

\section{Synthesis of 2-[(2-Benzenesulfonyl)vinyl]phenol}

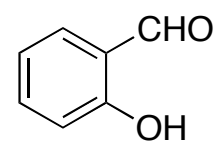

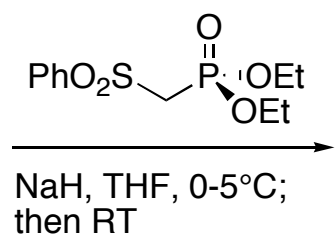

$\mathrm{NaH}, \mathrm{TH}$
then RT

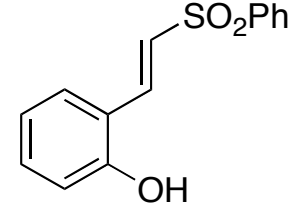

S3

Following the procedure outlined by Cheng et al., ${ }^{13}$ a suspension of sodium hydride $(95 \%, 0.900$ 
$\mathrm{g}, 37.5 \mathrm{mmol})$ was prepared in THF $(130 \mathrm{~mL})$ at $0{ }^{\circ} \mathrm{C}$. Benzenesulfonylmethylphosphonic acid diethyl ester $^{14}$ (14.6 g, $\left.50.0 \mathrm{mmol}\right)$ was added slowly to the stirred suspension, which transformed into a clear yellow solution. Salicylaldehyde $(2.66 \mathrm{~mL}, 25.0 \mathrm{mmol})$ was added dropwise using a syringe. The solution became a milky-yellow in color. The reaction mixture was stirred for an additional $2 \mathrm{~h}$ at room temperature, after which time it was quenched through the addition of water. The product was extracted with ethyl acetate, concentrated through rotary evaporation, and purified by column chromatography on a very large column (hexane/ethyl acetate, 1:1) to yield $\mathbf{S 3}\left(3.82 \mathrm{~g}, 58 \%\right.$ ) as a white solid: m.p. $156-162{ }^{\circ} \mathrm{C}$; IR (neat) $v_{\max } 3316$, 1603, 1581, 1499, 1444, 1365, $1288 \mathrm{~cm}^{-1} ;{ }^{1} \mathrm{H}$ NMR (500 MHz, acetone-d $) \delta 9.52$ (br s, 1H), 8.09-8.03 (m, 3H), 7.82-7.73 (m, 4H), 7.47 (d, $J=15.5 \mathrm{~Hz}, 1 \mathrm{H}), 7.43-7.39$ (m, 1H), 7.11 (dd, $J$ $=8.2,0.71 \mathrm{~Hz}, 1 \mathrm{H}), 7.02(\mathrm{tm}, J=8.1 \mathrm{~Hz}, 1 \mathrm{H}) ;{ }^{13} \mathrm{C}$ NMR $\left(125 \mathrm{MHz}\right.$, acetone-d $\left.\mathrm{d}_{6}\right) \delta 157.4,142.4$, 138.3, 133.6, 132.9, 130.7, 129.9 (2C), 128.4, 127.9 (2C), 120.6, 120.1, 116.7; HRMS (EI) calcd for $\mathrm{C}_{14} \mathrm{H}_{12} \mathrm{O}_{3} \mathrm{~S}\left[\mathrm{M}^{+}\right] 260.0507$, found 260.0508 .

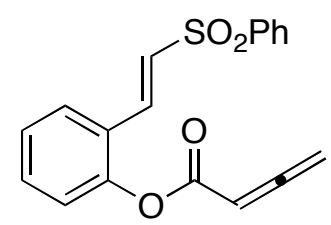

$1 \mathrm{i}$

2-[(2-Benzenesulfonyl)vinyl]phenyl Allenoate 1i. 53\% Yield; clear oil; IR (neat) $v_{\max } 3067$, 2993, 1967, 1931, 1731, 1616, 1482, $1447 \mathrm{~cm}^{-1} ;{ }^{1} \mathrm{H}$ NMR (500 MHz, $\left.\mathrm{CDCl}_{3}\right) \delta 7.96(\mathrm{~d}, J=8.2$ $\mathrm{Hz}, 2 \mathrm{H}), 7.81(\mathrm{~d}, J=15.5,1 \mathrm{H}), 7.65(\mathrm{td}, J=6.6,1.1,1 \mathrm{H}), 7.59-7.54(\mathrm{~m}, 3 \mathrm{H}), 7.46(\mathrm{tm}, J=7.8$ $\mathrm{Hz}, 1 \mathrm{H}), 7.30-7.26(\mathrm{~m}, 2 \mathrm{H}), 6.97(\mathrm{~d}, J=15.5 \mathrm{~Hz}, 1 \mathrm{H}), 5.85(\mathrm{t}, J=6.4 \mathrm{~Hz}, 1 \mathrm{H}), 5.45(\mathrm{~d}, J=6.4$ $\mathrm{Hz}, 2 \mathrm{H}) ;{ }^{13} \mathrm{C} \mathrm{NMR}\left(125 \mathrm{MHz}, \mathrm{CDCl}_{3}\right) \delta 217.0,163.2,149.4,140.3,136.2,133.4,131.9,129.5$, 129.3 (2C), 128.6, 127.6 (2C), 126.3, 125.0, 123.0, 87.1, 80.2; HRMS (EI) calcd for $\mathrm{C}_{18} \mathrm{H}_{14} \mathrm{O}_{4} \mathrm{~S}$ $\left[\mathrm{M}^{+}\right]$326.0613, found 326.0613.

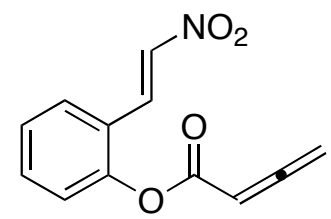

1j

Allenoate 1j. ${ }^{15} 67 \%$ Yield; pale-yellow solid; m.p. $74-78^{\circ} \mathrm{C}$; IR (film) $v_{\max } 1963,1940,1740$, 
1653, 1480, $1325 \mathrm{~cm}^{-1} ;{ }^{1} \mathrm{H}$ NMR $\left(500 \mathrm{MHz}, \mathrm{CDCl}_{3}\right) \delta 8.07(\mathrm{~d}, J=13.8 \mathrm{~Hz}, 1 \mathrm{H}), 7.69(\mathrm{~d}, J=$ $13.8 \mathrm{~Hz}, 1 \mathrm{H}), 7.58(\mathrm{dd}, J=7.7,1.4 \mathrm{~Hz}, 1 \mathrm{H}), 7.52$ (td, $J=8.5,1.6 \mathrm{~Hz}, 1 \mathrm{H}), 7.34-7.30(\mathrm{~m}, 2 \mathrm{H})$, $5.88(\mathrm{t}, 6.4 \mathrm{~Hz}, 1 \mathrm{H}) 5.48(\mathrm{~d}, J=6.5 \mathrm{~Hz}, 2 \mathrm{H}) ;{ }^{13} \mathrm{C} \mathrm{NMR}\left(125 \mathrm{MHz}, \mathrm{CDCl}_{3}\right) \delta 217.2,163.0,150.0$, $138.9,133.4,132.8,129.9,126.5,123.6,122.9,87.3,80.4$; MS (APCI) calcd for $\mathrm{C}_{12} \mathrm{H}_{9} \mathrm{NO}_{4}\left[\mathrm{M}^{+}\right]$ 232.1, found 232.1 .

\section{Phosphine-Catalyzed Annulation}

As an example of the typical experimental procedure used for the synthesis of the 1ethoxycarbonyl-2,9b-dihydro- $1 H$-cyclopena $[c]$ chromen-4-ones, the synthesis of 1ethoxycarbonyl-2,9b-dihydro-1H-cyclopena[c]chromen-4-one $\mathbf{2 a}$ is described.

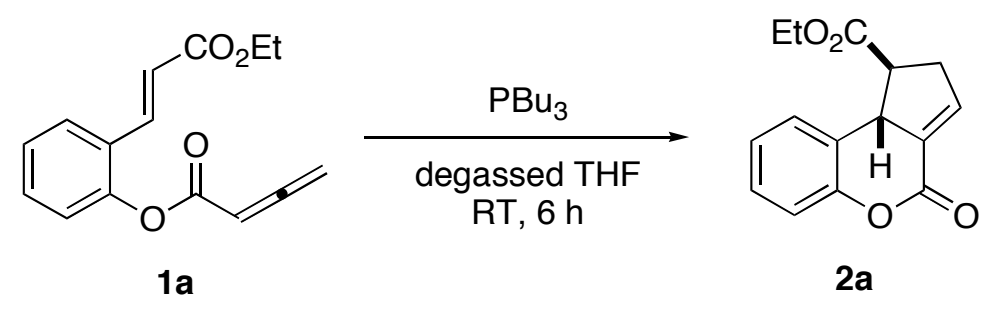

2-( $\beta$-Ethoxycarbonyl)styrenyl allenoate 1a $(323.0 \mathrm{mg}, 1.25 \mathrm{mmol})$ was dissolved in THF (200 $\mathrm{mL}$ ) in a 500-mL Schlenk flask charged with a stir bar. The solution was degassed using a freeze/pump/thaw method over three cycles and allowed to warm to room temperature. Tributylphosphine $(62.5 \mu \mathrm{L}, 0.25 \mathrm{mmol})$ was added quickly using a microsyringe. Within less than $10 \mathrm{~s}$, a color change from clear to yellow was observed in the stirred solution. Disappearance of the starting material was monitored using TLC and visualized through anisaldehyde staining. The product, which did not stain brightly with the anisaldehyde stain, was more clearly observed when using a permanganate stain. After $6 \mathrm{~h}$, the solution was concentrated through rotary evaporation. The crude residue was purified through column chromatography (hexane/ethyl acetate, 4:1) to yield 2a (310 mg, 96\% yield) as a clear oil; IR (neat) $v_{\max } 3067$, 2982, 1752, 1644, 1487, 1454, 1223, $1077 \mathrm{~cm}^{-1} ;{ }^{1} \mathrm{H}$ NMR $\left(500 \mathrm{MHz}, \mathrm{CDCl}_{3}\right) \delta 7.31(\mathrm{~d}, J=7.6$ $\mathrm{Hz}, 1 \mathrm{H}), 7.26$ (tm, $J=7.1 \mathrm{~Hz}, 1 \mathrm{H}), 7.15$ (tm, $J=7.5 \mathrm{~Hz}, 1 \mathrm{H}), 7.07$ (d, $J=8.1 \mathrm{~Hz}, 1 \mathrm{H}), 6.94-$ $6.92(\mathrm{~m}, 1 \mathrm{H}), 4.52-4.49(\mathrm{~m}, \mathrm{~Hz}, 1 \mathrm{H}), 4.31$ (q, $J=7.1 \mathrm{~Hz}, 2 \mathrm{H}), 3.45$ (app q, $J=9.3 \mathrm{~Hz}, 1 \mathrm{H}), 3.00$ (app dt, $J=9.0,3.1 \mathrm{~Hz}, 2 \mathrm{H}), 1.35(\mathrm{t}, J=7.1 \mathrm{~Hz}, 3 \mathrm{H}) ;{ }^{13} \mathrm{C} \mathrm{NMR}\left(125 \mathrm{MHz}, \mathrm{CDCl}_{3}\right) \delta 173.7$, $160.2,150.7,143.3,130.4,128.5,126.1,125.9,125.0$ 117.2, 61.5, 51.4, 45.7, 37.2, 14.2; HRMS 
(EI) calcd for $\mathrm{C}_{15} \mathrm{H}_{15} \mathrm{O}_{4}\left[(\mathrm{M}+\mathrm{H})^{+}\right]$259.0970, found 259.0963 .

The structure of 2a was assigned based on its 2D-NMR spectra. The NOESY, COSY, and HMQC spectra are provided below. For the $1 \mathrm{D}{ }^{1} \mathrm{H}$ and ${ }^{13} \mathrm{C}$ NMR spectra, please see the collection of spectra appended to this Supporting Information. The relative stereochemistry was assigned through comparison with the spectra of compound $\mathbf{2 i}$. For a full discussion of this analysis, refer to page S17. 
NOESY

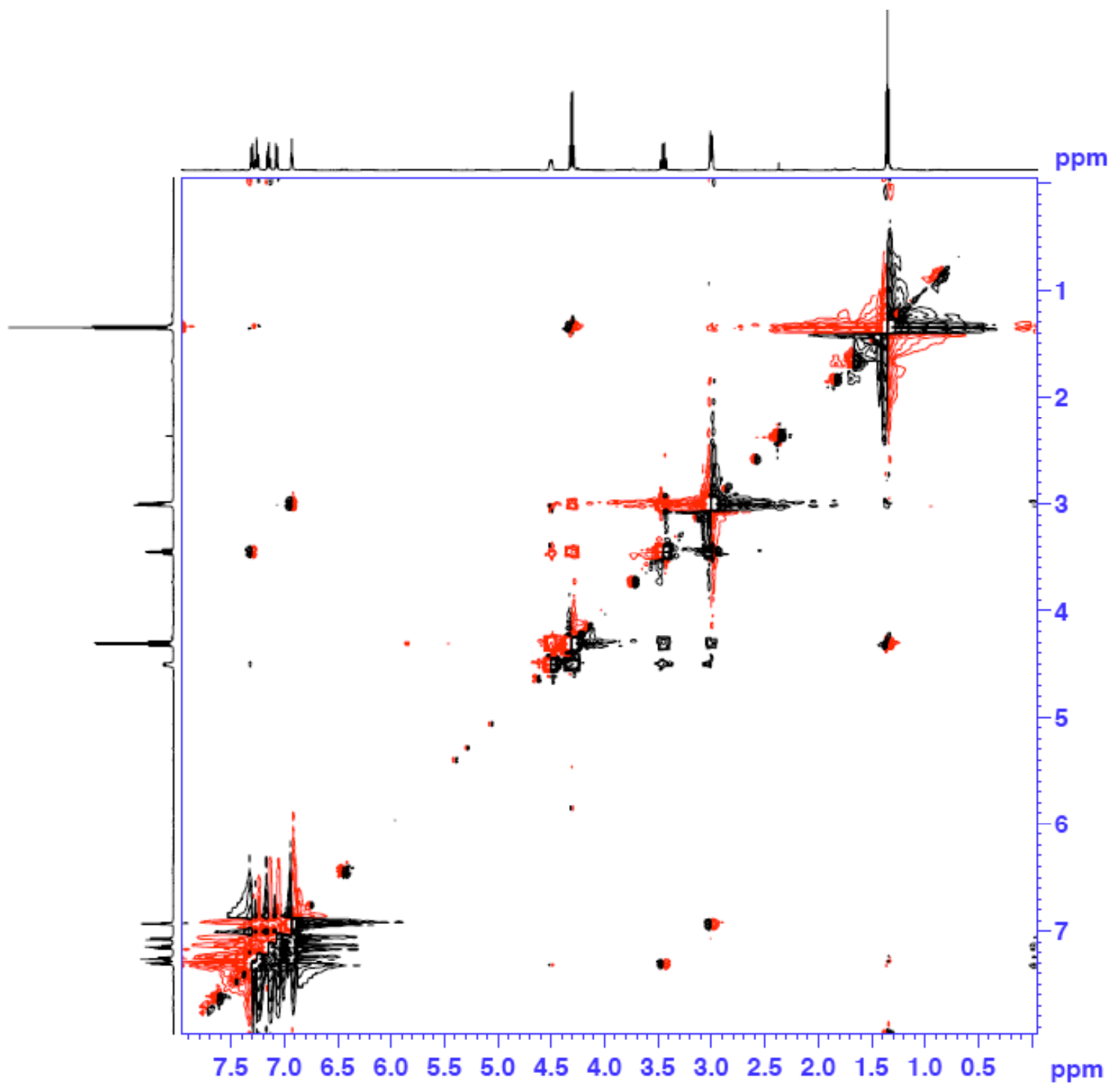


COSY

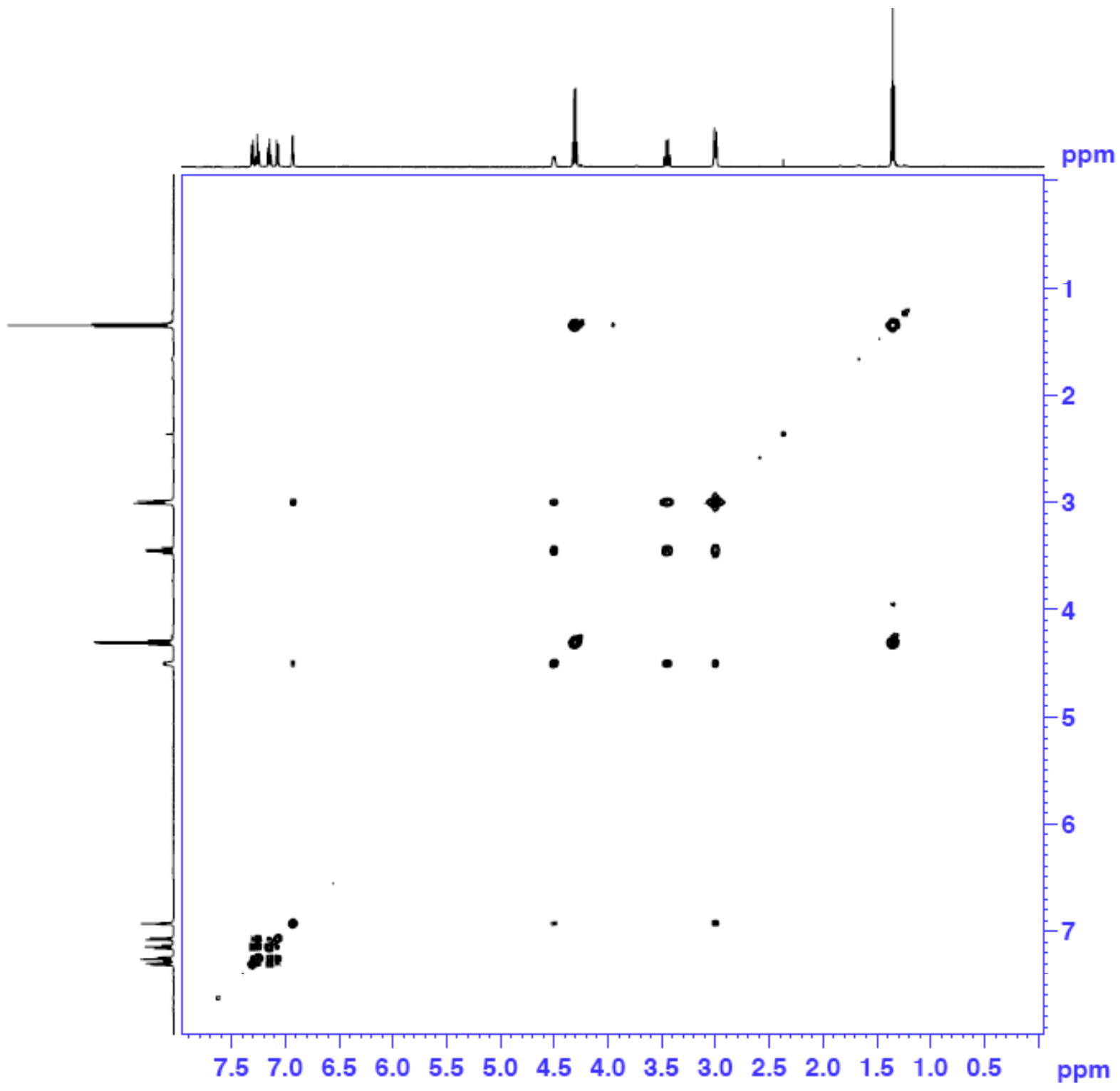


HMQC

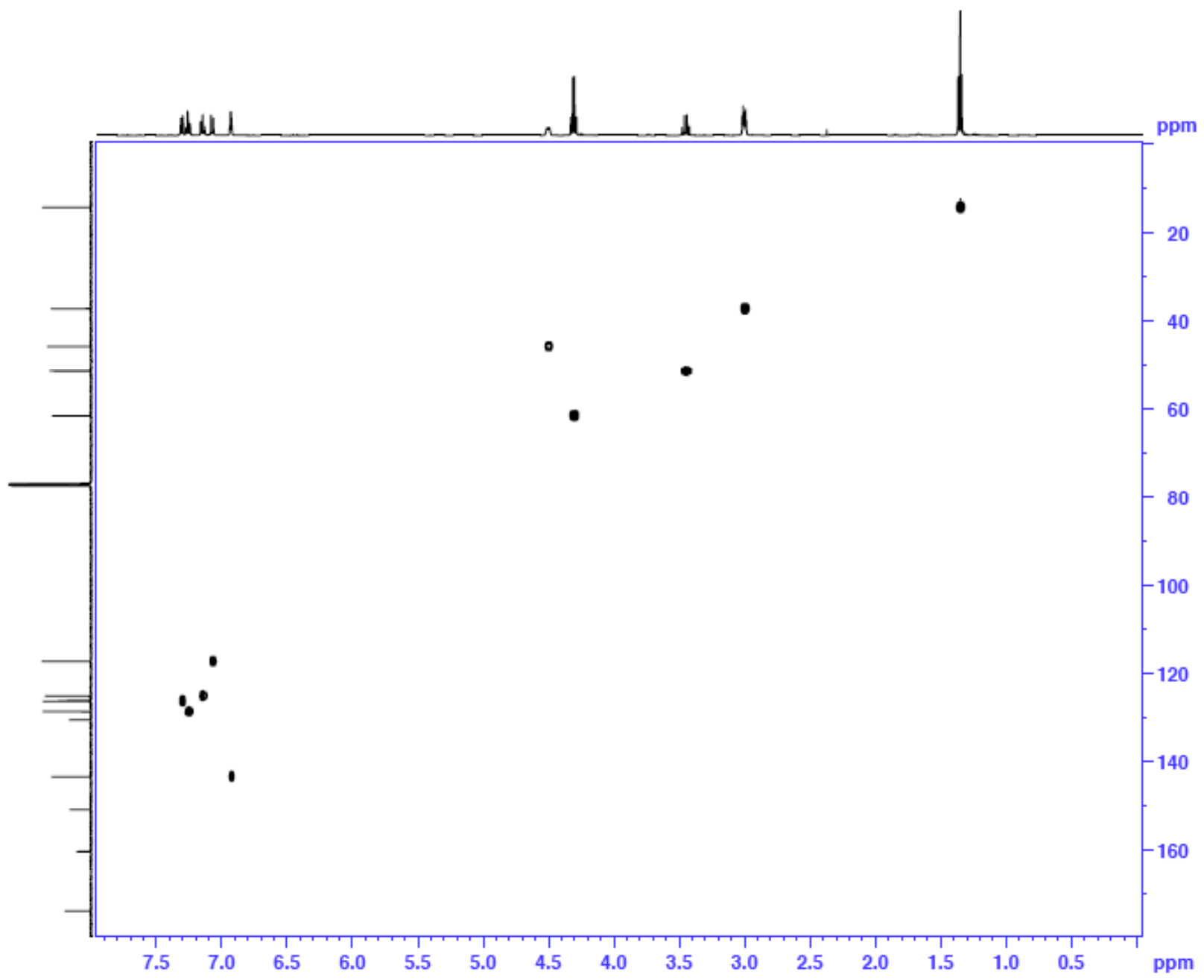




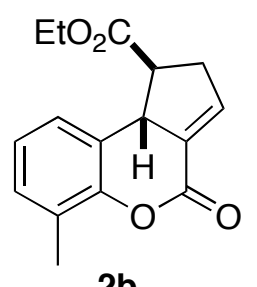

2b

1-Ethoxycarbonyl-2,9b-dihydro-1H-cyclopena/c/chromen-4-one 2b. 98\% Yield; clear oil; IR (neat) $v_{\max } 2981,1752,1732,1645,1464 \mathrm{~cm}^{-1} ;{ }^{1} \mathrm{H} \mathrm{NMR}\left(500 \mathrm{MHz}, \mathrm{CDCl}_{3}\right) \delta 7.13-7.09(\mathrm{~m}$, 2H), $7.03(\mathrm{t}, J=7.3 \mathrm{~Hz}, 1 \mathrm{H}), 6.91(\operatorname{app~q}, J=2.8 \mathrm{~Hz}, 1 \mathrm{H}), 4.48-4.50(\mathrm{~m}, 1 \mathrm{H}), 4.30$ (q, $J=7.1$ $\mathrm{Hz}, 2 \mathrm{H}), 3.44$ (q, $J=9.3 \mathrm{~Hz}, 1 \mathrm{H}), 3.00(\mathrm{dtm}, J=8.6,3.2,2 \mathrm{H}), 2.32(\mathrm{~s}, 3 \mathrm{H}), 1.34$ (t, $J=7.1 \mathrm{~Hz}$, $3 \mathrm{H}) ;{ }^{13} \mathrm{C}$ NMR $\left(125 \mathrm{MHz}, \mathrm{CDCl}_{3}\right) \delta 173.7,160.1,149.0,142.7,130.5,129.9,126.5,125.7$, 124.3, 123.4, 61.3, 51.3, 45.8, 37.2, 16.1 14.1; HRMS (EI) calcd for $\mathrm{C}_{16} \mathrm{H}_{16} \mathrm{O}_{4}\left[\mathrm{M}^{+}\right]$272.1049, found 272.1041 .

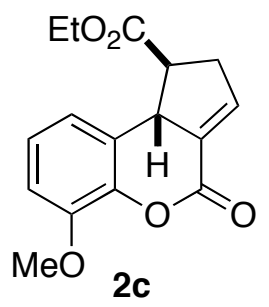

1-Ethoxycarbonyl-2,9b-dihydro-1H-cyclopena/c/chromen-4-one 2c. 74\% Yield; clear oil; IR (neat) $v_{\max } 2980,1750,1730,1644,1611,1583,1275,1174 \mathrm{~cm}^{-1} ;{ }^{1} \mathrm{H}$ NMR $\left(500 \mathrm{MHz}, \mathrm{CDCl}_{3}\right) \delta$ 7.08, (app t, $J=8.0 \mathrm{~Hz}, 1 \mathrm{H}), 6.92(\operatorname{app~q}, J=2.8 \mathrm{~Hz}, 1 \mathrm{H}), 6.87-6.85$ (m, 2H), 4.51-4.49 (m, Hz, $1 \mathrm{H}), 4.30$ (q, $J=7.1 \mathrm{~Hz}, 2 \mathrm{H}), 3.88(\mathrm{~s}, 3 \mathrm{H}), 3.44$ (q, $J=9.3 \mathrm{~Hz}, 1 \mathrm{H}), 3.01-2.97(\mathrm{~m}, 2 \mathrm{H}), 1.34$ (t, $J$ $=7.1 \mathrm{~Hz}, 3 \mathrm{H}) ;{ }^{13} \mathrm{C} \mathrm{NMR}\left(125 \mathrm{MHz}, \mathrm{CDCl}_{3}\right) \delta 173.6,159.4,147.7,143.0,140.0,130.2,126.9$, 124.8, 117.2, 111.3, 61.3, 56.0, 51.3, 45.8, 37.2, 14.1; HRMS (EI) calcd for $\mathrm{C}_{16} \mathrm{H}_{16} \mathrm{O}_{5}\left[\mathrm{M}^{+}\right]$ 288.0998, found 288.0996.

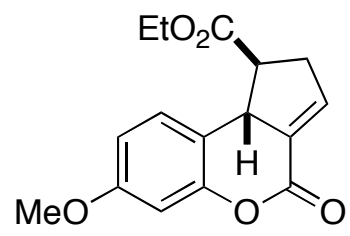

2d 
1-Ethoxycarbonyl-2,9b-dihydro-1H-cyclopena/c/chromen-4-one 2d. 94\% Yield; clear oil; IR (neat) $v_{\max } 2980,1751,1731,1624,1507,1232,1079 \mathrm{~cm}^{-1} ;{ }^{1} \mathrm{H}$ NMR (400 MHz, $\left.\mathrm{CDCl}_{3}\right) \delta 7.20$ $(\mathrm{dd}, J=8.5,1.1 \mathrm{~Hz}, 1 \mathrm{H}), 6.92-6.90(\mathrm{~m}, 1 \mathrm{H}), 6.69(\mathrm{dd}, J=8.5,2.6 \mathrm{~Hz}, 1 \mathrm{H}), 6.6(\mathrm{~d}, J=2.6 \mathrm{~Hz}$, $1 \mathrm{H}), 4.44-4.42(\mathrm{~m}, 1 \mathrm{H}), 4.31$ (q, $J=7.1 \mathrm{~Hz}, 2 \mathrm{H}), 3.77$ (s, 3H), 3.40 (app q, $J=9.2 \mathrm{~Hz}, 1 \mathrm{H})$, 3.00-2.94 (m, 2H), $1.34(\mathrm{t}, J=7.1 \mathrm{~Hz}, 3 \mathrm{H}) ;{ }^{13} \mathrm{C} \mathrm{NMR}\left(100 \mathrm{MHz}, \mathrm{CDCl}_{3}\right) \delta$ 173.7, 160.2, 159.7, $151.5,143.3,130.3,126.8,117.9,111.1,1102.6,61.5,55.6,51.8,45.1,37.1,14.3$; HRMS (EI) calcd for $\mathrm{C}_{16} \mathrm{H}_{16} \mathrm{O}_{5}\left[\mathrm{M}^{+}\right]$288.0998, found 288.0986.

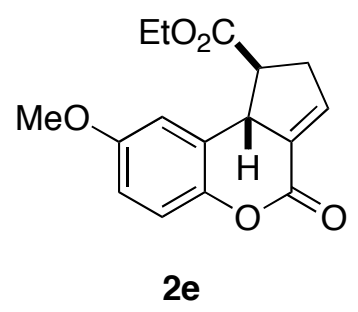

1-Ethoxycarbonyl-2,9b-dihydro-1H-cyclopena/c/chromen-4-one 2e. 70\% Yield; clear oil; IR (neat) $v_{\max } 2985,1734,1630,1079 \mathrm{~cm}^{-1} ;{ }^{1} \mathrm{H} \mathrm{NMR}\left(500 \mathrm{MHz}, \mathrm{CDCl}_{3}\right) \delta 7.01(\mathrm{~d}, J=8.9 \mathrm{~Hz}, 1 \mathrm{H})$, $6.93(\operatorname{app~q}, J=2.8 \mathrm{~Hz}, 1 \mathrm{H}), 6.89(\mathrm{dd}, J=2.9,1.2 \mathrm{~Hz}, 1 \mathrm{H}), 6.78(\mathrm{ddd}, J=8.9,3.0,0.8 \mathrm{~Hz}, 1 \mathrm{H})$, 4.49-4.46 (m, 1H), 4.31 (q, $J=7.1 \mathrm{~Hz}, 2 \mathrm{H}), 3.79$ (s, 3H), 3.45 (app q, $J=9.3 \mathrm{~Hz}, 1 \mathrm{H}), 3.02-$ $2.98(\mathrm{~m}, 2 \mathrm{H}), 1.36(\mathrm{t}, J=7.1 \mathrm{~Hz}, 3 \mathrm{H}) ;{ }^{13} \mathrm{C} \mathrm{NMR}\left(125 \mathrm{MHz}, \mathrm{CDCl}_{3}\right) \delta 173.5,160.2,156.5$, $144.7,143.1,130.3,126.8,117.8,113.2,111.4,61.4,55.5,51.3,45.9,37.0,14.2$; HRMS (EI) calcd for $\mathrm{C}_{16} \mathrm{H}_{16} \mathrm{O}_{5}\left[\mathrm{M}^{+}\right] 288.0998$, found 288.1006.

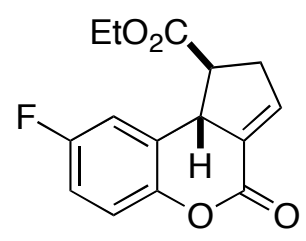

$2 f$

1-Ethoxycarbonyl-2,9b-dihydro-1H-cyclopena[c]chromen-4-one 2f. 91\% Yield; clear oil; IR (neat) $v_{\max } 3079,2982,1750,1731,1486,1433,1178,1154 \mathrm{~cm}^{-1} ;{ }^{1} \mathrm{H} \mathrm{NMR}\left(500 \mathrm{MHz}, \mathrm{CDCl}_{3}\right) \delta$ 7.07 (ddd, $J=8.6,2.9,1.2 \mathrm{~Hz}, 1 \mathrm{H}), 7.04$ (dd, $J=8.9,4.7 \mathrm{~Hz}, 1 \mathrm{H}), 6.97-6.92$ (m, 2H), 4.48-4.45 $(\mathrm{m}, 1 \mathrm{H}), 4.32$ (q, $J=7.1 \mathrm{~Hz}, 2 \mathrm{H}), 3.42(\operatorname{app~q}, J=9.3 \mathrm{~Hz}, 1 \mathrm{H}), 3.02(\mathrm{dt}, J=9.1,3.0 \mathrm{~Hz}, 2 \mathrm{H})$, $1.36(\mathrm{t}, J=7.1 \mathrm{~Hz}, 3 \mathrm{H}) ;{ }^{13} \mathrm{C} \mathrm{NMR}\left(125 \mathrm{MHz}, \mathrm{CDCl}_{3}\right) \delta 173.3,159.7,159.4$, (d, $\left.J_{\mathrm{CF}}=244.1 \mathrm{~Hz}\right)$, 146.8, 144.0, 129.5, 127.6, 118.5, $115.2\left(\mathrm{~d}, J_{\mathrm{CF}}=23.3 \mathrm{~Hz}\right), 113.0\left(\mathrm{~d}, J_{\mathrm{CF}}=24.4 \mathrm{~Hz}\right), 61.7,51.3$, 
45.8, 37.0, 14.2; HRMS (EI) calcd for $\mathrm{C}_{15} \mathrm{H}_{13} \mathrm{FO}_{4}\left[\mathrm{M}^{+}\right]$276.0798, found 276.0795.

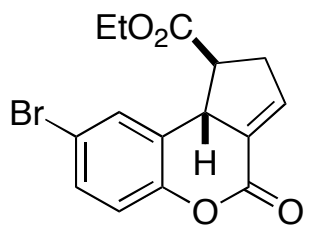

$2 g$

1-Ethoxycarbonyl-2,9b-dihydro-1H-cyclopena/c]chromen-4-one 2g. 93\% Yield; clear oil; IR (neat) $v_{\max } 3074,2982,1732,1644,1475,1407,1178 \mathrm{~cm}^{-1} ;{ }^{1} \mathrm{H}$ NMR $\left(500 \mathrm{MHz}, \mathrm{CDCl}_{3}\right) \delta 7.48-$ 7.49 (m, 1H), 7.37 (ddd, $J=8.7,2.3,0.8 \mathrm{~Hz}, 1 \mathrm{H}), 6.97-6.94$ (m, 2H), 4.46-4.48 (m, 1H), 4.26 (q, $J=7.1 \mathrm{~Hz}, 2 \mathrm{H}), 3.44(\operatorname{app~q}, J=9.3 \mathrm{~Hz}, 1 \mathrm{H}), 3.00(\operatorname{app~dt}, 9.0,3.1 \mathrm{~Hz}, 2 \mathrm{H}), 1.37$ (t, $J=7.1 \mathrm{~Hz}$, $3 \mathrm{H}) ;{ }^{13} \mathrm{C}$ NMR $\left(125 \mathrm{MHz}, \mathrm{CDCl}_{3}\right) \delta 173.2,159.4,149.8,144.2,131.5,129.4,129.3,128.0$, 118.9, 117.6, 61.7, 51.2, 45.6, 37.0, 14.2; HRMS (EI) calcd for $\mathrm{C}_{15} \mathrm{H}_{13}{ }^{79} \mathrm{BrO}_{4}\left[\mathrm{M}^{+}\right]$335.9997, found 335.9992; calcd for $\mathrm{C}_{15} \mathrm{H}_{13}{ }^{81} \mathrm{BrO}_{4}\left[\mathrm{M}^{+}\right]$337.9977, found 337.9969.

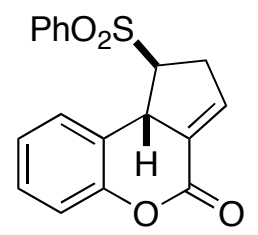

2i

1-Benzenesulfonyl-2,9b-dihydro-1H-cyclopena/c]chromen-4-one 2i. $63 \%$ Yield; clear oil; IR (neat) $v_{\max } 1751,1650,1486,1451,1146,1071 \mathrm{~cm}^{-1} ;{ }^{1} \mathrm{H} \mathrm{NMR}\left(500 \mathrm{MHz}, \mathrm{CDCl}_{3}\right) \delta 8.06(\mathrm{~d}, J=$ $8.0 \mathrm{~Hz}, 2 \mathrm{H}), 7.77$ (t, $J=7.1 \mathrm{~Hz}, 1 \mathrm{H}), 7.69$ (t, $J=7.7 \mathrm{~Hz}, 2 \mathrm{H}), 7.51$ (d, $J=7.6 \mathrm{~Hz}, 1 \mathrm{H}), 7.32$ (t, $J$ $=7.6 \mathrm{~Hz}, 1 \mathrm{H}), 7.20(\mathrm{t}, J=7.6 \mathrm{~Hz}, 1 \mathrm{H}), 7.11(\mathrm{~d}, J=8.1 \mathrm{~Hz}, 1 \mathrm{H}), 6.82-6.79(\mathrm{~m}, 1 \mathrm{H}), 4.83-4.80$ (m, 1H), 4.27 (q, $J=8.7,7.8 \mathrm{~Hz}, 1 \mathrm{H}), 3.23$ (ddt, $J=19.0,7.3,2.4 \mathrm{~Hz}, 1 \mathrm{H}), 2.82$ (ddt, $J=19.0$, 9.3, $3.0 \mathrm{~Hz}, 1 \mathrm{H}) ;{ }^{13} \mathrm{C} \mathrm{NMR}\left(125 \mathrm{MHz}, \mathrm{CDCl}_{3}\right) \delta$ 159.7, 150.7, 141.0, 137.6, 134.4, 130.2, 129.7 (2C), 128.9, 128.3 (2C), 126.5, 125.2, 124.4, 117.3, 69.2, 43.8, 36.1; HRMS (EI) calcd for $\mathrm{C}_{18} \mathrm{H}_{15} \mathrm{O}_{4} \mathrm{~S}\left[(\mathrm{M}+\mathrm{H})^{+}\right]$327.0691, found 327.0699 . 


\section{Stereochemistry of tricyclic dihydrocoumarin 2: 2D-NMR of 2i}

The expected trans stereochemistry was confirmed through 2D NMR spectroscopic studies.

Compound $2 \mathbf{i}$ was used for determination of the stereochemistry because its diastereotopic protons $\mathrm{H}^{\mathrm{c}}$ and $\mathrm{H}^{\mathrm{c}^{\prime}}$ were resolved and had distinct $\delta$ values. The characteristic ${ }^{1} \mathrm{H}$ NMR splitting patterns for the cyclopentene ring protons were conserved across all derivatives 2 (Figure S1). In the NOESY spectrum of $\mathbf{2 i}$ (next page), protons $\mathrm{H}^{\mathrm{a}}$ and $\mathrm{H}^{\mathrm{e}}$ exhibited an NOE to proton $\mathrm{H}^{\mathrm{c}}$, but not to proton $\mathrm{H}^{\mathrm{c}^{\prime}}$. Complete 2D NMR spectra for compound $\mathbf{2} \mathbf{i}$ are included on the following pages. For the $1 \mathrm{D}{ }^{1} \mathrm{H}$ and ${ }^{13} \mathrm{C}$ NMR spectra of compound $\mathbf{2} \mathbf{i}$, see the attached compilation of spectral data.

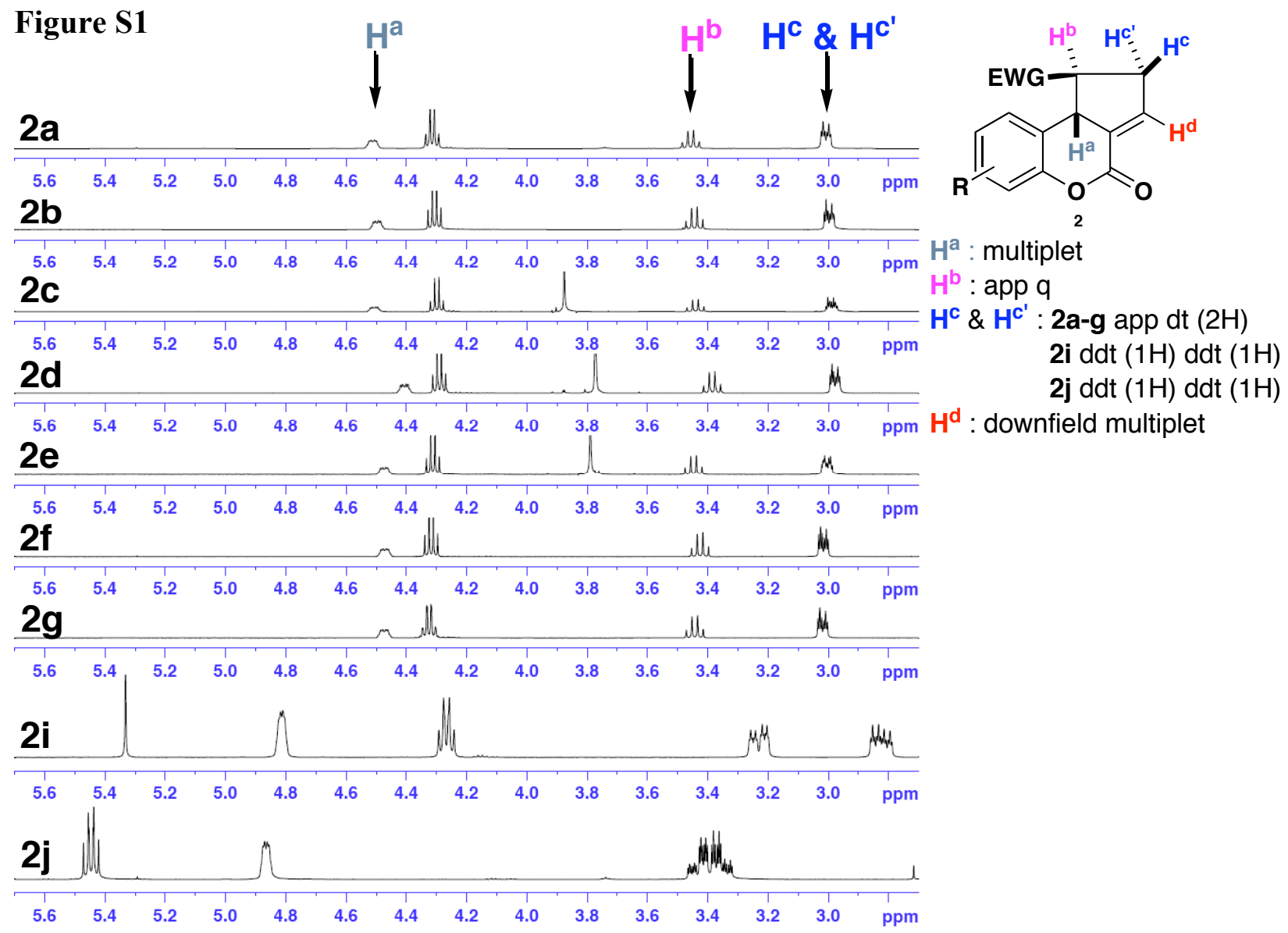


Henry and Kwon:

NOSEY
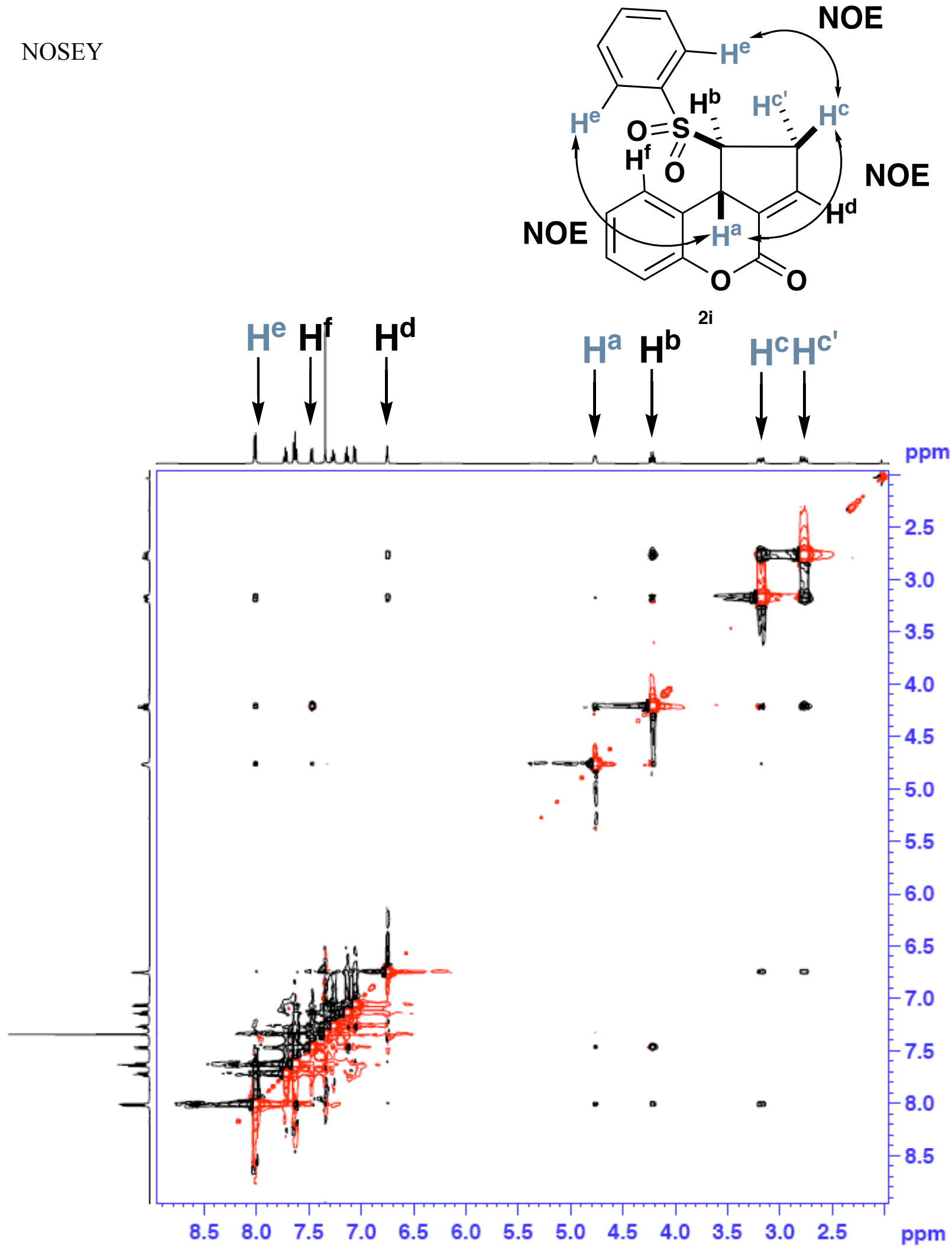
COSY

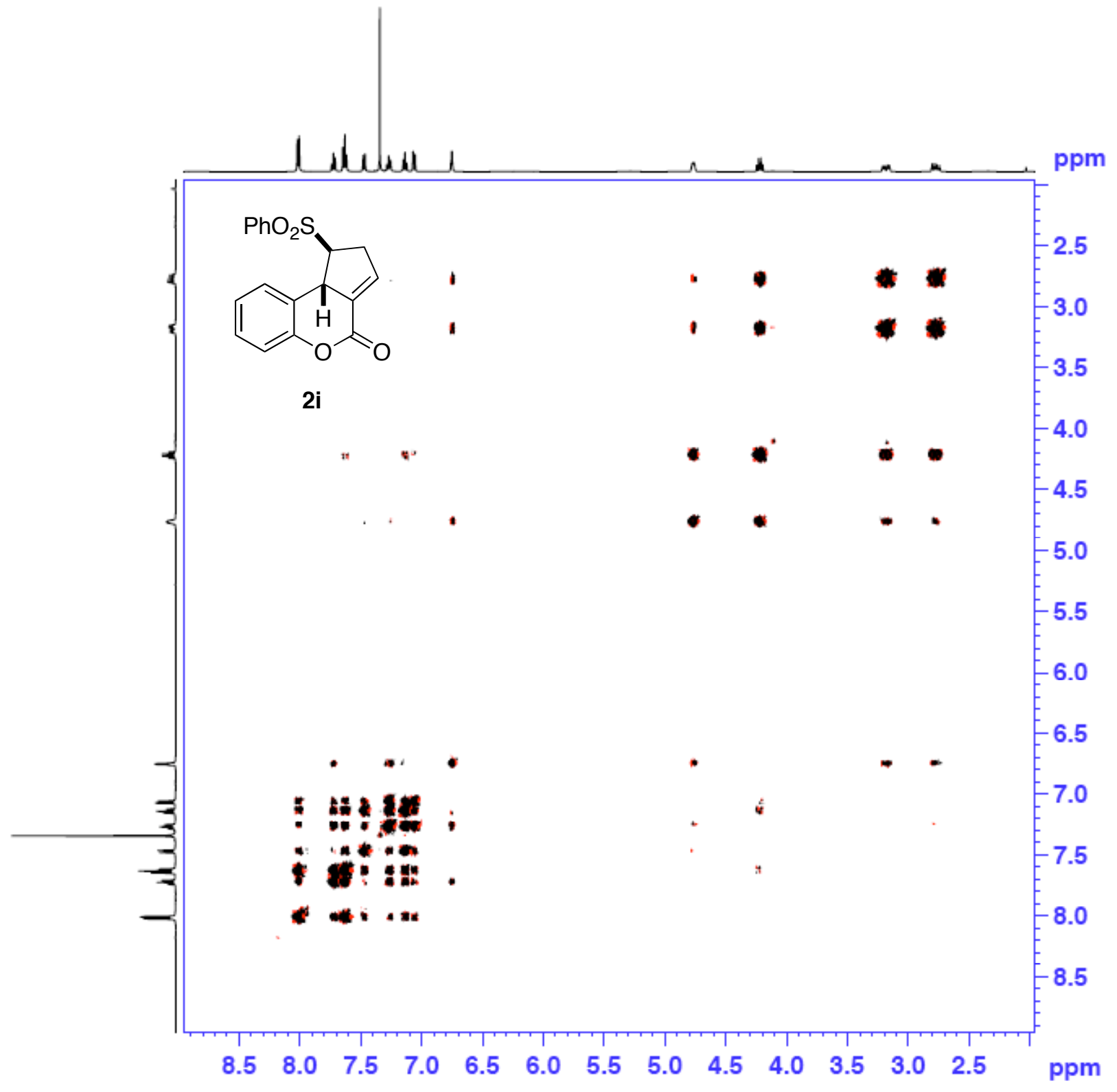




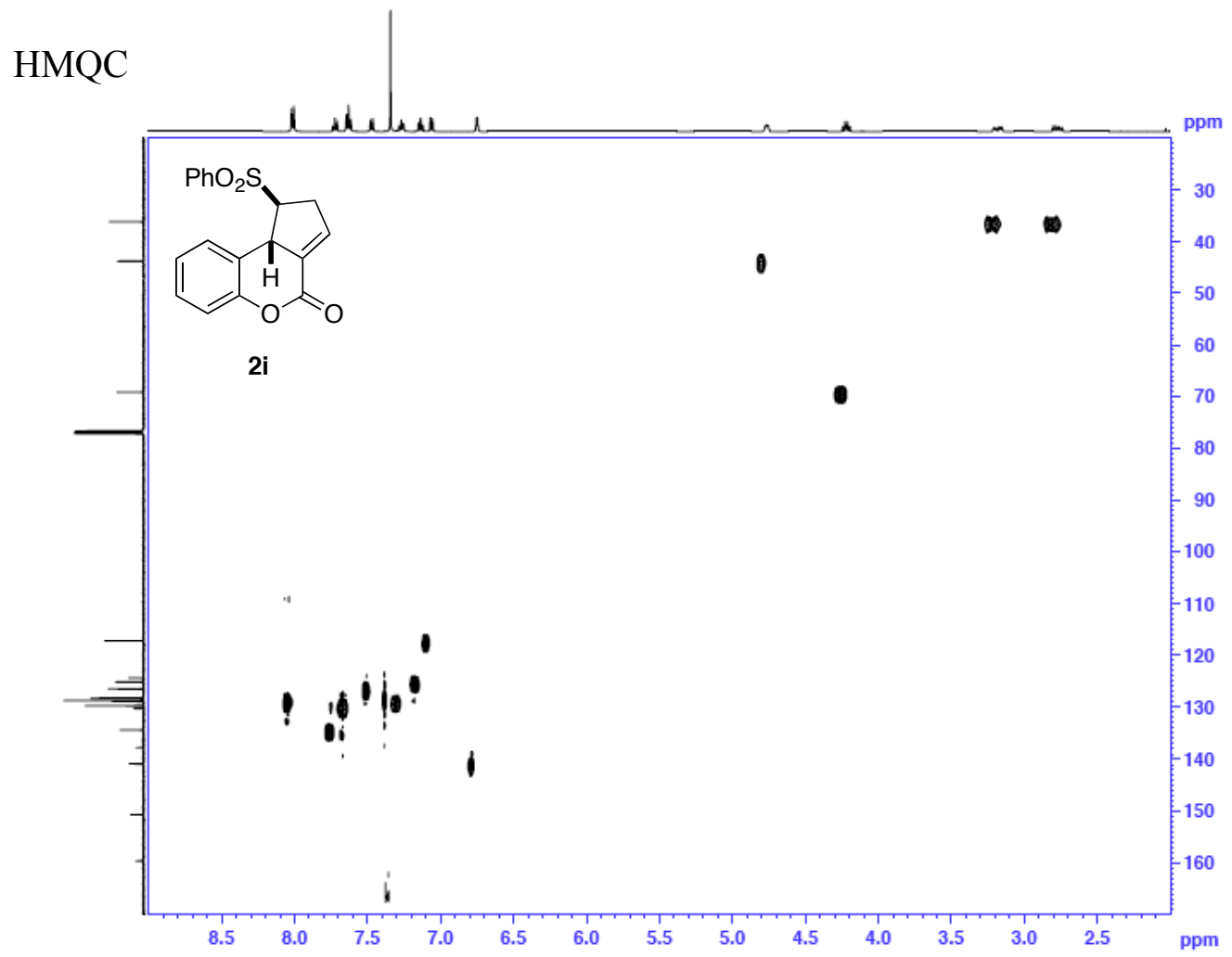

\section{HMBC}

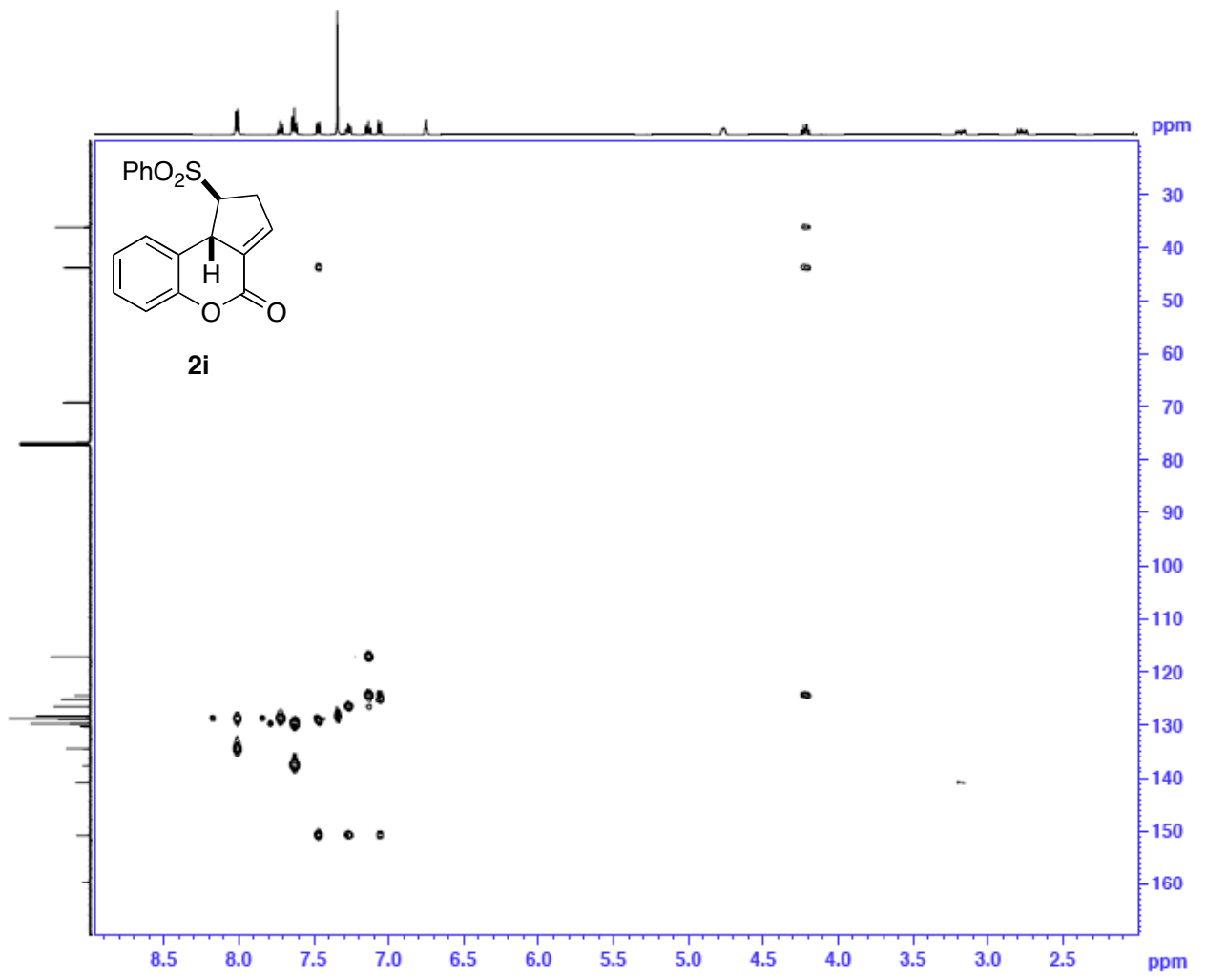




\section{Reactions of the $\beta$-Nitrostyrenyl Derivatives: Screening of the Phosphine Catalyst}

Subjecting allenoate $\mathbf{1 j}$ to the reaction conditions established for the $\alpha, \beta$-unsaturated ester systems and the $\alpha, \beta$-unsaturated sulfone system led to only a trace amount of product being visible in the ${ }^{1} \mathrm{H}$ NMR spectra of the crude material (Table S1, entry 1). Accordingly, a number of commercially available phosphines were screened in an attempt to afford a higher yield. Among the phosphines screened, triphenylphosphine provided the best yield of $\mathbf{2 j}$ (48\%; Table S1, entry 5). Interestingly, a second compound was observed under these conditions; its structure was established to be that of the nitronate 3 .<smiles>C=CC(=O)Oc1ccccc1/C=C/[N+](=O)[O-]</smiles>

$1 \mathrm{j}$

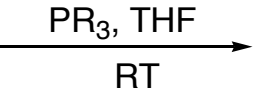

RT<smiles>C[C@@]12C(=CC[C@H]1[N+](=O)[O-])C(=O)Oc1ccccc12</smiles>

2j<smiles>CC1O[N+]([O-])=Cc2c1c(=O)oc1ccccc21</smiles>

3

Table S1. Phosphine Screen for Reaction of Allenoate 1j

\begin{tabular}{llllll}
\hline entry & phosphine & temp $\left({ }^{\circ} \mathrm{C}\right)$ & time $(\mathrm{h})$ & yield $^{\mathrm{a}}$ of $\mathbf{2 j}$ & yield $^{\mathrm{a}}$ of $\mathbf{3}$ \\
\hline 1 & tributylphosphine & $\mathrm{RT}$ & 24 & trace & 0 \\
2 & trimethylphosphine & $\mathrm{RT}$ & 5 & 0 & 0 \\
3 & tricyclopentylphosphine & $\mathrm{RT}$ & 5 & 0 & 0 \\
4 & triisopropylphosphine & $\mathrm{RT}$ & 5 & 0 & 0 \\
5 & triphenylphosphine & $\mathrm{RT}$ & 5 & $48 \%$ & $12 \%$ \\
& Tri( $p$-fluorophenyl)phosphine & $\mathrm{RT}$ & 5 & $49 \%$ & $11 \%$ \\
$6^{\mathrm{b}}$ & tri( $o$-tolyl)phosphine & $\mathrm{RT}$ to reflux & $48+$ & 0 & 0 \\
$7^{\mathrm{b}}$ & $(p$-dimethylaminophenyl)diphenylphosphine & $\mathrm{RT}$ & 6 & $13 \%$ & $2 \%$ \\
$8^{\mathrm{b}}$ & tris $(p$-dimethylaminophenyl)phosphine & $\mathrm{RT}$ to reflux & $48+$ & 0 & 0 \\
$9^{\mathrm{b}}$ & tris(2,4,6-trimethoxyphenyl)phosphine & RT to reflux & $48+$ & 0 & 0 \\
\hline
\end{tabular}

${ }^{\mathrm{a}}$ Isolated yield. ${ }^{\mathrm{b}}$ The major reaction observed was hydrolysis to give $o$-hydroxy- $\beta$-nitrostyrene $\mathbf{1} \mathbf{j}$.

\section{Screening of Solvents for the Phosphine-Catalyzed Reactions of Allenoate 1j}

Still unsatisfied with the yield provided above, a number of common organic solvents were screened for the reaction of allenoate $\mathbf{1 j}$ with triphenylphosphine. None afforded an increased yield of $\mathbf{2} \mathbf{j}$, but, interestingly, benzene provided a reversal in the product ratios such that the nitronate 3 was obtained in 58\% yield (Table S2, entry 5). Use of HMPT led to degradation of the starting allenoate, while trimethylphosphite provided no observable reaction. Use of tri( $p$ fluorophenyl)phosphine provided a slight increase in the yield of the nitronate 3 (62\%; Table S2, entry 8). 
<smiles>C=CCC(=O)Oc1ccccc1/C=C/[N+](=O)[O-]</smiles>

$1 \mathbf{j}$
$\underset{\mathrm{RT}}{\stackrel{\mathrm{PR}_{3}, \text { solvent }}{\longrightarrow}}$<smiles>C[C@@]12C(=CCC1[N+](=O)[O-])C(=O)Oc1ccccc12</smiles>

2j<smiles>CC1O[N+]([O-])=Cc2c1c(=O)oc1ccccc21</smiles>

3

Table S2. Solvents Screened for Phosphine Catalysis of Allenoate 1j

\begin{tabular}{lllllll}
\hline entry & phosphine & solvent & temp $\left({ }^{\circ} \mathrm{C}\right)$ & time $(\mathrm{hr})$ & $\begin{array}{l}\text { yield }^{\mathrm{a}}(\%) \text { of } \\
\mathbf{2 j}\end{array}$ & $\begin{array}{l}\text { yield }^{\mathbf{a}}(\%) \text { of } \\
\mathbf{3}\end{array}$ \\
\hline 1 & triphenylphosphine & $\mathrm{THF}$ & $\mathrm{RT}$ & 5 & 48 & 12 \\
2 & triphenylphosphine & $\mathrm{Et} 2$ & $\mathrm{RT}$ & 5 & 40 & 10 \\
3 & triphenylphosphine & $\mathrm{CH}_{2} \mathrm{Cl}_{2}$ & $\mathrm{RT}$ & 5 & 13 & 2 \\
4 & triphenylphosphine & $\mathrm{CHCl}_{3}$ & $\mathrm{RT}$ & 5 & 27 & 23 \\
5 & triphenylphosphine & Benzene & $\mathrm{RT}$ & 5 & 14 & 58 \\
6 & trimethylphosphite & Benzene & $\mathrm{RT}$ & 12 & 0 & 0 \\
7 & hexamethylphosphortriamide & Benzene & $\mathrm{RT}$ & - & - & - \\
8 & tri(p-fluorophenyl)phosphine & Benzene & $\mathrm{RT}$ & 5 & 19 & 62 \\
\hline
\end{tabular}

${ }^{\mathrm{a}}$ Isolated yield.

Spectral data for $\mathbf{2} \mathbf{j}$ and nitronate $\mathbf{3}$ are provided:

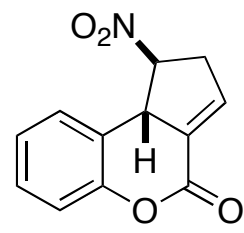

2j

1-Nitro-2,9b-dihydro-1H-cyclopena/c/chromen-4-one 2j. 48\% Yield; clear oil; IR (neat) $v_{\max }$ 1751, 1646, 1550, 1487, 1454, $1370 \mathrm{~cm}^{-1} ;{ }^{1} \mathrm{H}$ NMR (500 MHz, $\left.\mathrm{CDCl}_{3}\right) \delta 7.38-7.31(\mathrm{~m}, 2 \mathrm{H})$, $7.21(\mathrm{td}, J=7.5,1.1 \mathrm{~Hz}, 1 \mathrm{H}), 7.10(\mathrm{dd}, J=8.2,1.0 \mathrm{~Hz}, 1 \mathrm{H}), 6.91$ (app q, $J=3.0 \mathrm{~Hz}, 1 \mathrm{H}), 5.44$ (app q, $J=8.0 \mathrm{~Hz}, 1 \mathrm{H}), 4.88-4.85(\mathrm{~m}, 1 \mathrm{H}), 3.43(\mathrm{ddtd}, J=18.8,8.0,2.9,0.4 \mathrm{~Hz}, 1 \mathrm{H}), 3.35$ (ddt, $J=18.7,8.6,2.8,1 \mathrm{H}) ;{ }^{13} \mathrm{C}$ NMR $\left(125 \mathrm{MHz}, \mathrm{CDCl}_{3}\right) \delta 158.8,150.6,140.5,129.5,128.8,125.7$, 125.4, 123.0, 117.6, 90.4, 47.5, 38.5; HRMS (EI) calcd for $\mathrm{C}_{12} \mathrm{H}_{10} \mathrm{NO}_{4}\left[(\mathrm{M}+\mathrm{H})^{+}\right] 232.0610$, found 232.0620 . 


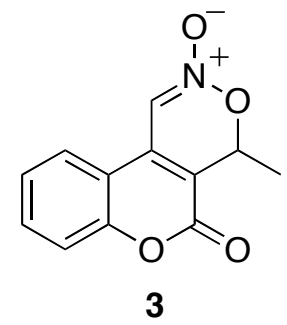

Nitronate 3. $62 \%$ Yield; yellow solid; m.p. ca. $200{ }^{\circ} \mathrm{C}$ (decomp.); IR (film) $v_{\max } 3084,2923$, 1726, 1630, 1608, $1564 \mathrm{~cm}^{-1} ;{ }^{1} \mathrm{H}$ NMR $\left(500 \mathrm{MHz}, \mathrm{CDCl}_{3}\right) \delta 7.64-7.36(\mathrm{~m}, 1 \mathrm{H}), 7.53(\mathrm{dd}, J=$ 7.9, $1.2 \mathrm{~Hz}, 1 \mathrm{H}), 7.48(\mathrm{~s}, 1 \mathrm{H}), 7.43(\mathrm{~d}, J=7.6 \mathrm{~Hz}, 1 \mathrm{H}), 7.38$ (td, $J=7.7,0.9 \mathrm{~Hz}, 1 \mathrm{H}), 5.76(\mathrm{q}, J=$ $6.7 \mathrm{~Hz}, 1 \mathrm{H}), 1.67(\mathrm{~d}, J=6.7 \mathrm{~Hz}, 3 \mathrm{H}) ;{ }^{13} \mathrm{C} \mathrm{NMR}\left(125 \mathrm{MHz}, \mathrm{CDCl}_{3}\right) \delta 157.7,153.6,133.1,132.7$, 125.0, 122.5, 117.9, 113.9, 113.7, 106.7, 77.0, 17.2; HRMS (MALDI) calcd for $\mathrm{C}_{12} \mathrm{H}_{10} \mathrm{NO}_{4}[(\mathrm{M}$ $\left.+\mathrm{H})^{+}\right] 232.0604$, found 232.0605 .

\section{1,3-dipolar cycloadditions of nitronate 3}

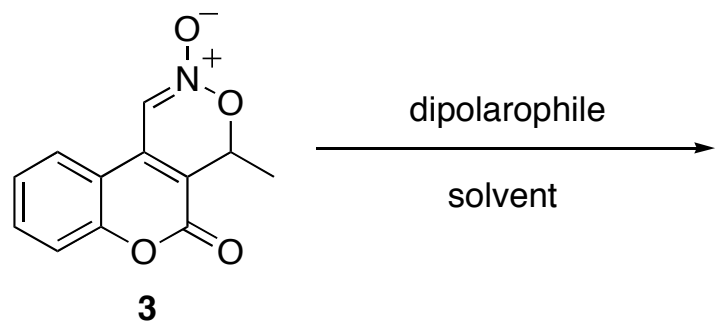<smiles>[X]C1ON2O[C@@H](C)c3c(c(=O)oc4ccccc34)[C@@]2([2H])C1[R7]</smiles>

4<smiles>[X]C1ON2OC(C)c3c(c(=O)oc4ccccc34)C2(C)C1[R]</smiles>

5

Table S3. Synthesis of Nitrosoacetals 4 and 5 Through 1,3-Dipolar Cycloaddition

\begin{tabular}{|c|c|c|c|c|c|c|}
\hline entry & dipolarophile & solvent & temp $\left({ }^{\circ} \mathrm{C}\right)$ & time $(\mathrm{h})$ & yield $^{\mathrm{a}}(\%)$ & $4 / 5$ \\
\hline 1 & & toluene & 50 & 18 & 98 & $2: 1$ \\
\hline 2 & & dichloromethane & RT & 40 & 97 & $7: 1$ \\
\hline 3 & & toluene & RT & 26 & 93 & $9: 1$ \\
\hline 4 & & toluene & 50 & 26 & 95 & $9: 1$ \\
\hline 5 & & toluene & RT & 14 & $0^{\mathrm{b}}$ & n.a. \\
\hline 6 & & toluene & 90 & 48 & $0^{\mathrm{b}}$ & n.a. \\
\hline 7 & & dichloromethane & RT & 4 & 94 & $11: 1$ \\
\hline 8 & & dichloromethane & RT & 15 & 83 & $10: 1$ \\
\hline 9 & & dichloromethane & RT & $72+$ & $\mathrm{NR}^{\mathrm{c}}$ & n.a. \\
\hline
\end{tabular}

\footnotetext{
${ }^{\mathrm{a}}$ Isolated yield. ${ }^{\mathrm{b}}$ Complex mixture of products; none identified as nitrosoacetals. ${ }^{\mathrm{c}}$ Mostly recovered starting material.
} 
As an example of the typical procedure used for the 1,3-dipolar cycloaddition reactions of nitronate $\mathbf{3}$ leading to the nitrosoacetals $\mathbf{4 a}-\mathbf{e}$, the synthesis of nitrosoacetal $\mathbf{4} \mathbf{c}$ is provided:

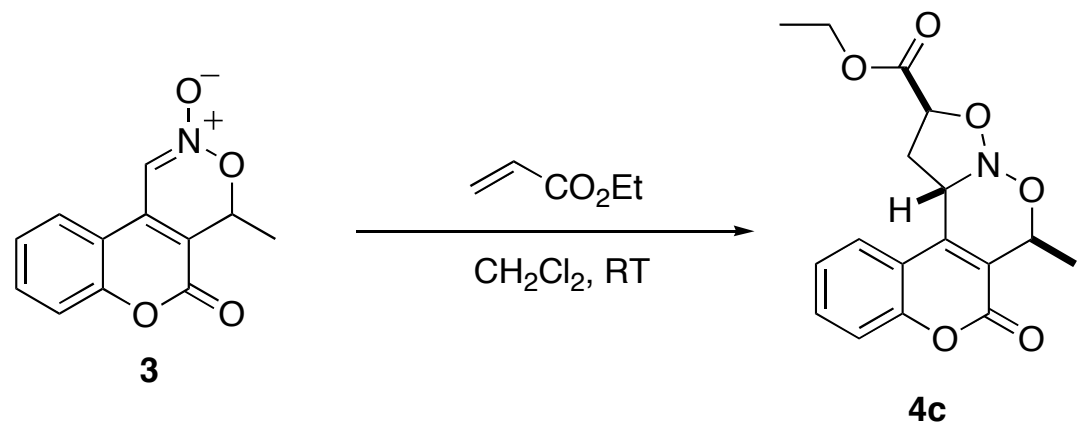

Nitronate 3 (83.3 $\mathrm{mg}, 0.36 \mathrm{mmol})$ was dissolved in a mixture of ethyl acrylate and dichloromethane $(1: 1,6 \mathrm{~mL})$. The stirred solution was monitored for $4 \mathrm{~h}$ using TLC, after which time the starting nitronate was no longer observed. The solution was concentrated through rotary evaporation and the crude residue was then purified by column chromatography (hexane/ethyl acetate, $6: 1)$ to yield the nitrosoacetal $4 \mathrm{c}$ (113 $\mathrm{mg}, 86 \%, 94 \%$ combined) as a crystalline solid; IR (neat) $v_{\max } 2985,1720,1633,1608,1453,1210 \mathrm{~cm}^{-1} ;{ }^{1} \mathrm{H}$ NMR $\left(500 \mathrm{MHz}, \mathrm{CDCl}_{3}\right) \delta 7.58-7.55$ $(\mathrm{m}, 1 \mathrm{H}), 7.46(\mathrm{dd}, J=7.9,1.3 \mathrm{~Hz}, 1 \mathrm{H}), 7.40(\mathrm{dd}, J=9.1,0.8 \mathrm{~Hz}, 1 \mathrm{H}), 7.36(\mathrm{td}, J=7.9,0.8 \mathrm{~Hz}$, $1 \mathrm{H}), 5.21(\mathrm{dd}, J=9.9,3.5 \mathrm{~Hz}, 1 \mathrm{H}), 5.04(\mathrm{q}, J=6.6 \mathrm{~Hz}, 1 \mathrm{H}), 4.67$ (dd, $J=10.6,8.0 \mathrm{~Hz}, 1 \mathrm{H})$, 4.34-4.28 (m, 2H), 2.98-2.93 (m, 1H), 2.63 (dt, $J=11.5,10.3 \mathrm{~Hz} 1 \mathrm{H}), 1.73$ (d, $J=6.6 \mathrm{~Hz}, 3 \mathrm{H})$, $1.36(\mathrm{t}, J=7.1 \mathrm{~Hz}, 3 \mathrm{H}) ;{ }^{13} \mathrm{C} \mathrm{NMR}\left(125 \mathrm{MHz}, \mathrm{CDCl}_{3}\right) \delta 169.8,158.5,153.1,142.6,131.7,124.8$, 124.7, 122.9, 117.4, 81.6, 73.1, 65.0, 62.1, 34.7, 18.5, 14.1; HRMS (EI) calcd for $\mathrm{C}_{17} \mathrm{H}_{18} \mathrm{NO}_{6}$ $\left[(\mathrm{M}+\mathrm{H})^{+}\right]$332.1134, found 332.1161.

Spectral data for the nitrosoacetals $\mathbf{4 a - e}$ are provided for all of the major diastereoisomers and also for the minor diastereoisomer of the nitrosoacetal 5a. X-Ray crystal structures are provided for nitrosoacetals 4a-major, 5a-minor, and $\mathbf{4 d}$. 


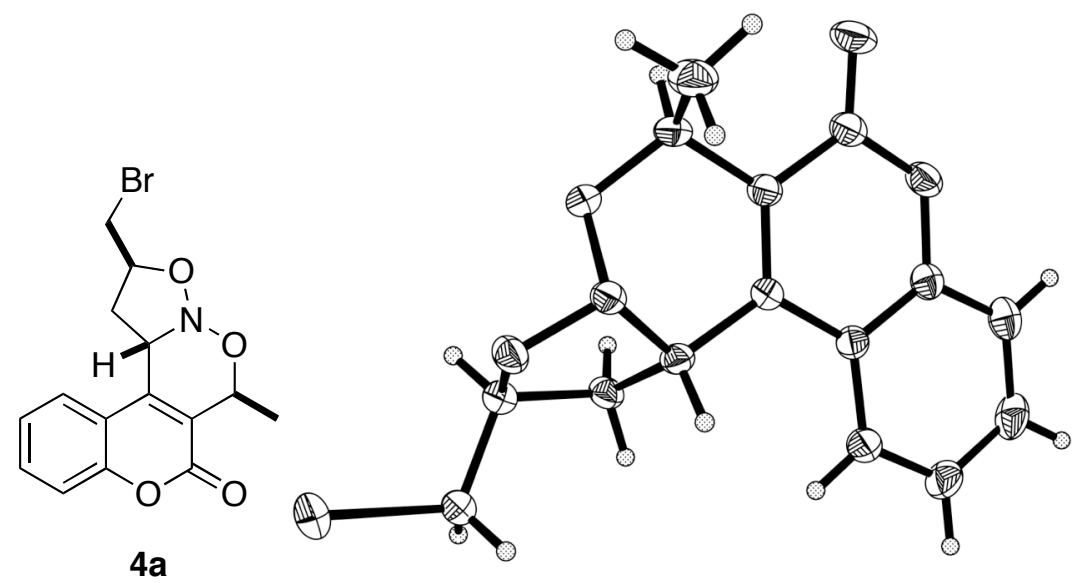

Nitrosoacetal 4a-major. 85\% Yield (97\% Combined Yield); crystalline solid; m.p. $134-136{ }^{\circ} \mathrm{C}$; IR (neat) $v_{\max } 2936,1718,1632,1607,1574,14521395 \mathrm{~cm}^{-1} ;{ }^{1} \mathrm{H}$ NMR $\left(500 \mathrm{MHz}, \mathrm{CDCl}_{3}\right) \delta$ $7.59-7.55(\mathrm{~m}, 1 \mathrm{H}), 7.45(\mathrm{dd}, J=7.9,1.4 \mathrm{~Hz}, 1 \mathrm{H}), 7.40-7.34(\mathrm{~m}, 2 \mathrm{H}), 5.11-5.01(\mathrm{~m}, 1 \mathrm{H}), 5.03$ $(\mathrm{q}, J=6.6 \mathrm{~Hz}, 1 \mathrm{H}), 4.67(\mathrm{dd}, J=10.3,8.6 \mathrm{~Hz}, 1 \mathrm{H}), 3.73(\mathrm{dd}, J=11.4,4.3 \mathrm{~Hz}, 1 \mathrm{H}), 3.68(\mathrm{dd}, J=$ 11.4, 3.3 Hz, 1H), 2.71 (ddd, $J=12.1,8.4,3.8 \mathrm{~Hz}, 1 \mathrm{H}), 2.54-2.46$ (ddd, $J=11.2,11.1,9.3 \mathrm{~Hz}$, 1H), $1.72(\mathrm{~d}, J=6.6 \mathrm{~Hz}, 3 \mathrm{H}) ;{ }^{13} \mathrm{C} \mathrm{NMR}\left(125 \mathrm{MHz}, \mathrm{CDCl}_{3}\right) \delta 158.6,153.1,143.3,131.7,124.8$, 124.4, 122.9, 117.5, 117.4, 82.2, 72.8, 65.4, 35.3, 34.2, 18.5; HRMS (MALDI) calcd for $\mathrm{C}_{15} \mathrm{H}_{15} \mathrm{BrNO}_{4}\left[(\mathrm{M}+\mathrm{H})^{+}\right]$352.0184, found 352.0194.

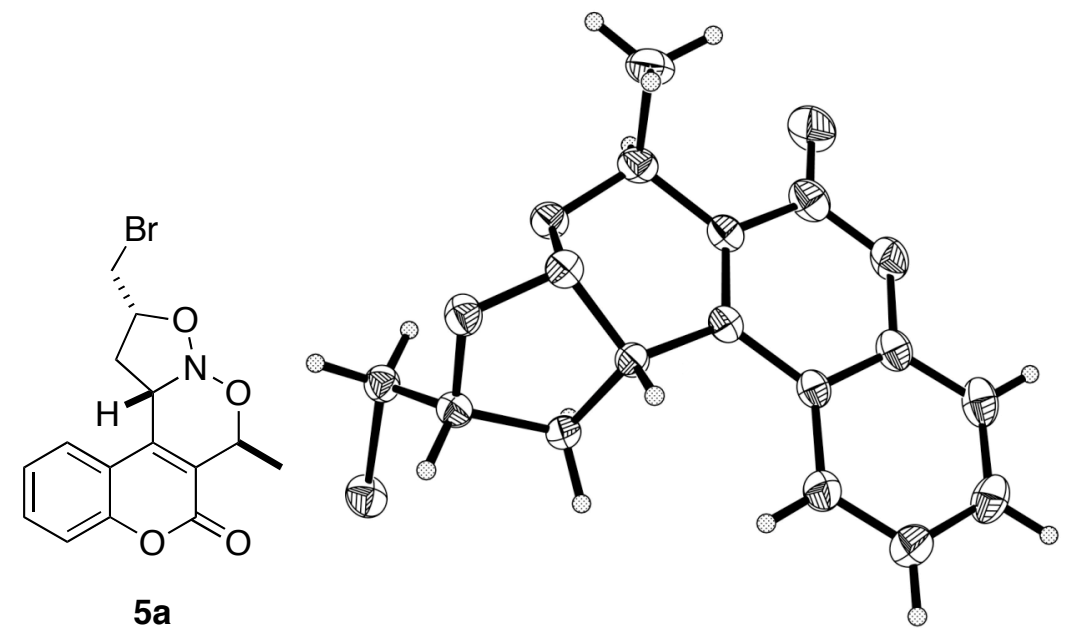

Nitrosoacetal 5a-minor. 12\% Yield (97\% Combined Yield); crystalline solid; m.p. $132-133{ }^{\circ} \mathrm{C}$; IR (neat) $v_{\max } 1713,1634,1452,1396 \mathrm{~cm}^{-1} ;{ }^{1} \mathrm{H} \mathrm{NMR}\left(500 \mathrm{MHz}, \mathrm{CDCl}_{3}\right) \delta$ 7.59-7.55 (m, 1H), $7.46(\mathrm{dd}, J=7.9,1.3 \mathrm{~Hz}, 1 \mathrm{H}), 7.40(\mathrm{dd}, J=8.5,0.7 \mathrm{~Hz}, 1 \mathrm{H}), 7.37-7.33(\mathrm{~m}, 1 \mathrm{H}), 5.03(\mathrm{q}, J=6.6$ $\mathrm{Hz}, 1 \mathrm{H}), 4.97-4.91(\mathrm{~m}, 1 \mathrm{H}), 4.58(\mathrm{dd}, J=11.2,7.7 \mathrm{~Hz}, 1 \mathrm{H}), 3.74(\mathrm{dd}, J=9.6,4.7 \mathrm{~Hz}, 1 \mathrm{H}), 3.51$ 
( app t, $J=9.8 \mathrm{~Hz}, 1 \mathrm{H}), 3.10$ (app dt, $J=11.7,7.7 \mathrm{~Hz}, 1 \mathrm{H}), 2.32-2.25(\mathrm{~m}, 1 \mathrm{H}), 1.72(\mathrm{~d}, J=6.6$ $\mathrm{Hz}, 3 \mathrm{H}) ;{ }^{13} \mathrm{C}$ NMR $\left(125 \mathrm{MHz}, \mathrm{CDCl}_{3}\right) \delta 158.6,153.1,142.8,131.7,124.7,124.7,122.7,117.49$, $117.45,86.1,73.2,65.9,37.0,34.7,18.4$; HRMS (MALDI) calcd for $\mathrm{C}_{15} \mathrm{H}_{15} \mathrm{BrNO}_{4}\left[(\mathrm{M}+\mathrm{H})^{+}\right]$ 352.0184 , found 352.0196 .

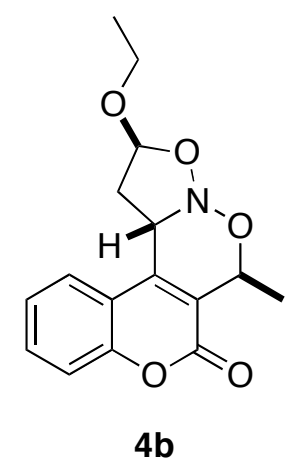

Nitrosoacetal 4b. 85\% Yield (93\% Combined Yield); clear oil; IR (neat) $v_{\max } 1720,1607,1452$, $1107 \mathrm{~cm}^{-1} ;{ }^{1} \mathrm{H}$ NMR (500 MHz, d $\mathrm{d}_{6}$-acetone) $\delta 7.77(\mathrm{dd}, J=7.9,1.3 \mathrm{~Hz}, 1 \mathrm{H}), 7.68-7.64(\mathrm{~m}, 1 \mathrm{H})$, 7.46-7.44 (m, 1H), 7.40 (dd, $J=8.3,0.8 \mathrm{~Hz}, 1 \mathrm{H}), 5.83$ (dd, $J=6.1,1.2 \mathrm{~Hz}, 1 \mathrm{H}), 4.87-4.79$ (m, $2 \mathrm{H}), 3.85-3.79(\mathrm{~m}, 1 \mathrm{H}), 3.68-3.61(\mathrm{~m}, 1 \mathrm{H}), 2.66(\mathrm{ddd}, J=12.5,7.1,1.3 \mathrm{~Hz}, 1 \mathrm{H}), 2.57$ (ddd, $J=$ 12.4, 11.2, $6.2 \mathrm{~Hz}, 1 \mathrm{H}), 1.62(\mathrm{~d}, J=6.6 \mathrm{~Hz}, 3 \mathrm{H}), 1.23(\mathrm{t}, J=7.1 \mathrm{~Hz}, 3 \mathrm{H}) ;{ }^{13} \mathrm{C}$ NMR $(125 \mathrm{MHz}$, $\mathrm{d}_{6}$-acetone) $\delta 158.8,153.7,144.1,132.2,125.4,125.2,124.6,118.4,117.4,109.9,72.8,65.1$, 63.9, 37.6, 18.5, 15.3; HRMS (EI) calcd for $\mathrm{C}_{16} \mathrm{H}_{18} \mathrm{NO}_{5}\left[(\mathrm{M}+\mathrm{H})^{+}\right]$304.1185, found 304.1194.

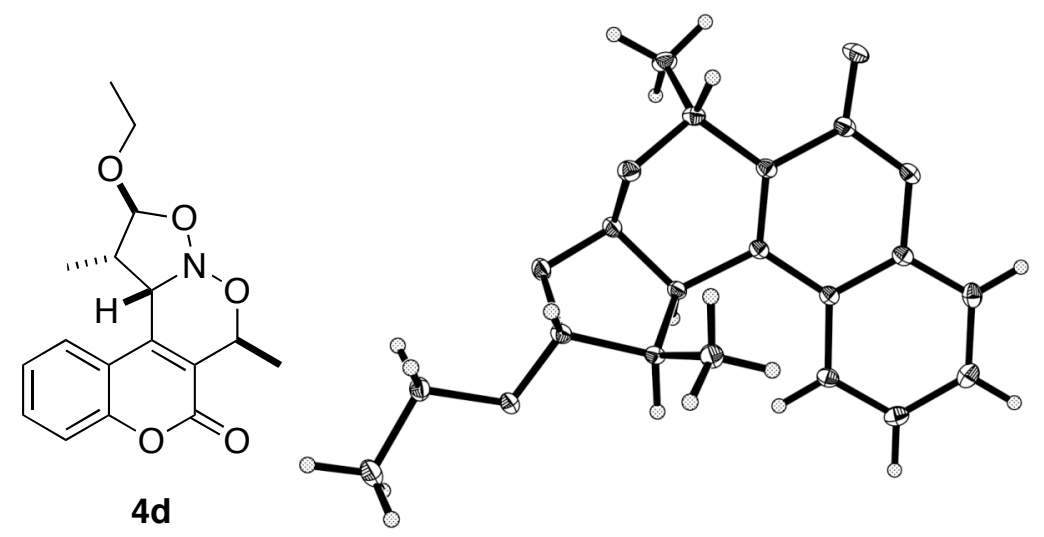

Nitrosoacetal 4d. $86 \%$ Yield (95 \% Combined Yield); crystalline solid: m.p. $141-144{ }^{\circ} \mathrm{C}$; IR (neat) $v_{\max } 2940,1722,1612,1448 \mathrm{~cm}^{-1} ;{ }^{1} \mathrm{H}$ NMR $\left(500 \mathrm{MHz}, \mathrm{CDCl}_{3}\right) \delta 7.57-7.53(\mathrm{~m}, 1 \mathrm{H}), 7.47$ (dd, $J=7.9,1.3 \mathrm{~Hz}, 1 \mathrm{H}), 7.39(\mathrm{dd}, J=8.3,0.9 \mathrm{~Hz}, 1 \mathrm{H}), 7.35-7.31(\mathrm{~m}, 1 \mathrm{H}), 5.58(\mathrm{~d}, J=2.1 \mathrm{~Hz}$, 
1H), 4.99 (q, $J=6.6 \mathrm{~Hz}, 1 \mathrm{H}), 4.81$ (d, $J=8.5 \mathrm{~Hz}, 1 \mathrm{H}), 3.94-3.87$ (m, 1H), 3.67-3.61 (m, 1H), 2.97-2.95 (m, 1H), $1.69(\mathrm{~d}, J=6.6 \mathrm{~Hz}, 3 \mathrm{H}), 1.28(\mathrm{t}, J=7.1 \mathrm{~Hz}, 3 \mathrm{H}), 1.05(\mathrm{~d}, J=7.5 \mathrm{~Hz}, 3 \mathrm{H})$; ${ }^{13} \mathrm{C}$ NMR $\left(125 \mathrm{MHz}, \mathrm{CDCl}_{3}\right) \delta 158.5,152.8,141.4,131.6,126.3,124.8,122.8,117.2,117.2$, 116.7, 72.4, 66.4, 65.7, 43.7, 18.4, 15.3, 14.9; HRMS (EI) calcd for $\mathrm{C}_{17} \mathrm{H}_{20} \mathrm{NO}_{5}\left[(\mathrm{M}+\mathrm{H})^{+}\right]$ 318.1341 , found 318.1325 .

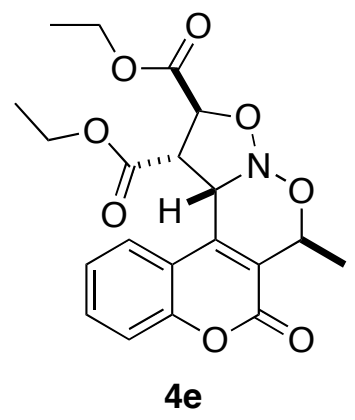

Nitrosoacetal 4e. $75 \%$ Yield (83\% Combined Yield); solid; m.p. 153-157 ${ }^{\circ} \mathrm{C}$; IR (neat) $v_{\max }$ 2984, 1723, 1630, $1450 \mathrm{~cm}^{-1} ;{ }^{1} \mathrm{H}$ NMR (500 MHz, CDCl $)$ 7.59-7.50 (m, 2H), 7.40-7.30 (m, 2H), 5.84-5.83 (m, 1H), 4.98-4.94 (m, 1H), 4.86 (d, $J=9.0 \mathrm{~Hz}, 1 \mathrm{H}), 4.33$ (q, $J=7.1 \mathrm{~Hz}, 2 \mathrm{H})$, 4.07-4.04 (m, 1H), 3.90-3.87 (m, 1H), 3.80-3.77 (m, 1H), $1.71(\mathrm{~d}, J=6.6 \mathrm{~Hz}, 3 \mathrm{H}), 1.36(\mathrm{t}, J=$ $7.1 \mathrm{~Hz}, 3 \mathrm{H}), 0.71(\mathrm{t}, J=7.1 \mathrm{~Hz}, 3 \mathrm{H}) ;{ }^{13} \mathrm{C} \mathrm{NMR}\left(125 \mathrm{MHz}, \mathrm{CDCl}_{3}\right) \delta$ 168.8, 167.7, 158.3, 152.6, 140.7, 131.6, 125.3, 124.7, 123.3, 118.1, 117.2, 83.7, 73.4, 67.6, 62.5, 61.6, 50.6, 18.3, 14.1, 13.2; HRMS (EI) calcd for $\mathrm{C}_{20} \mathrm{H}_{21} \mathrm{NO}_{8}\left[(\mathrm{M}+\mathrm{H})^{+}\right]$404.1345, found 404.1337.

\section{References:}

${ }^{1}$ Bunce, R. A.; Schilling, C. L. Tetrahedron 1997, 53, 9477-9486.

2 Takeuchi, Y.; Uedo, N.; Uesugi, K.; Abe, H.; Nishioka, H.; Harayama, T. Heterocycles 2003, 59, 217-224.

${ }^{3}$ Bunce, R. A.; Moore, J. D. Org. Prep. Proced. Int. 1997, 29, 293-299.

${ }^{4}$ Yamazaki, J.; Watanabe, T.; Tanaka, K. Tetrahedron: Asymmetry 2001, 12, 669-675.

${ }^{5}$ Coste, J.; Frerot, E.; Jouin, P. J. Org. Chem. 1994, 59, 2437-2446.

${ }^{6}$ Caddick, S.; Hamza, D.; Wadman, S. N. Tetrahedron Lett. 1999, 40, 7285-7288.

${ }^{7}$ Rockwell, A.; Melden, M.; Copeland, R. A.; Hardman, K.; Decicco, C. P.; Degrado, W. F. J. Am. Chem. Soc. 1996, 118, 10337-10338.

${ }^{8}$ Giacomelli, G.; Porcheddu, A.; Salaris, M. Org. Lett. 2003, 5, 2715-2717.

9 (a) Ishihara, K.; Ohara, S.; Yamamoto, H. Science 2000, 290, 1140-1142. (b) Ishihara, K.; Nakayama, M.; Ohara, S.; Yamamoto, H. Tetrahedron 2002, 58, 8179-8188.

${ }^{10}$ Hashimoto, S.; Furukawa, I. Bull. Chem. Soc. Jpn. 1981, 54, 2227-2228. 
11 (a) Mukaiyama, T.; Usui, M.; Shimada, E. Chem. Lett. 1975, 1045-1048. (b) Mukaiyama, T.; Toda, H.; Susumu, K. Chem. Lett. 1976, 13-14. (c) Sparks, S. M.; Gutierrez, A. J.; Shea, K. J. J. Org. Chem. 2003, 68, 5274-5285.

12 Bennasar, M.-L.; Zulaica, E.; Juan, C.; Alonso, Y.; Bosch, J. J. Org. Chem. 2002, 67, $7465-7474$.

${ }^{13}$ Cheng, J. J.; Cheng, J.-M.; Roush, W. R. Org. Lett. 2003, 5, 1967-1970.

14 (a) Enders, D.; von Ber, S.; Jandeleit, B. Org. Synth. Col. Vol. 10, p 289-292; Vol. 78, p 169-172. (b) Shahak, L.; Almog, J. Synthesis 1969, 170-172. (c) Shahak, I.; Almog, J.

Synthesis 1970, 145-146.

${ }^{15}$ For the synthesis of the precursor phenol via Henry reaction, see: Dowd, C. S.; Herric-Davis, K.; Egan, C.; Dupre, A.; Smith, C.; Milt, T.; Glennon, R. A. J. Med. Chem. 2000, 43, 3074. 


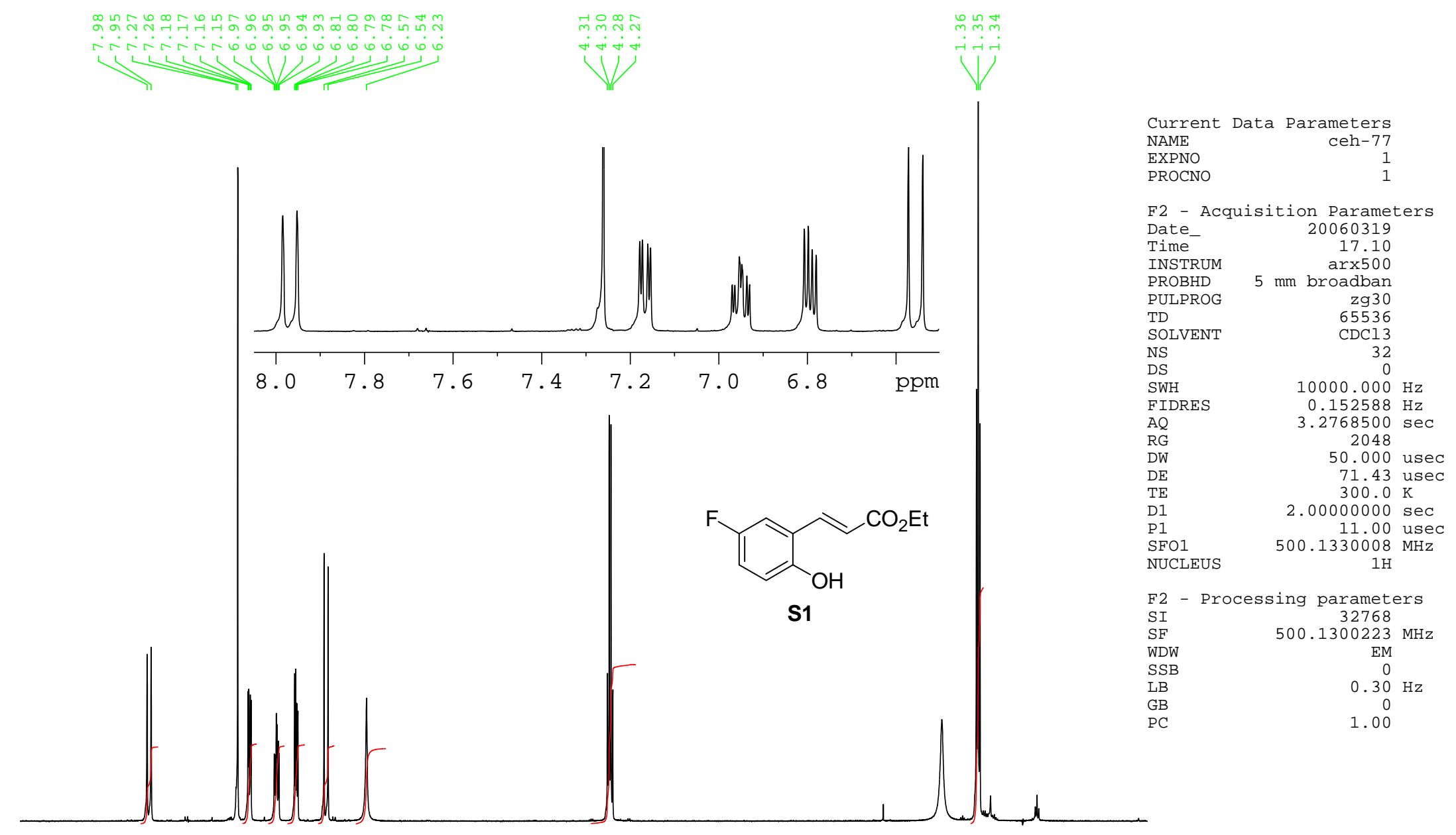

$\begin{array}{lllllllllllllllll}8.5 & 8.0 & 7.5 & 7.0 & 6.5 & 6.0 & 5.5 & 5.0 & 4.5 & 4.0 & 3.5 & 3.0 & 2.5 & 2.0 & 1.5 & 1.0 & \text { ppm }\end{array}$

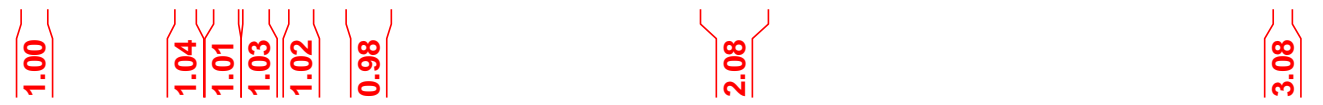




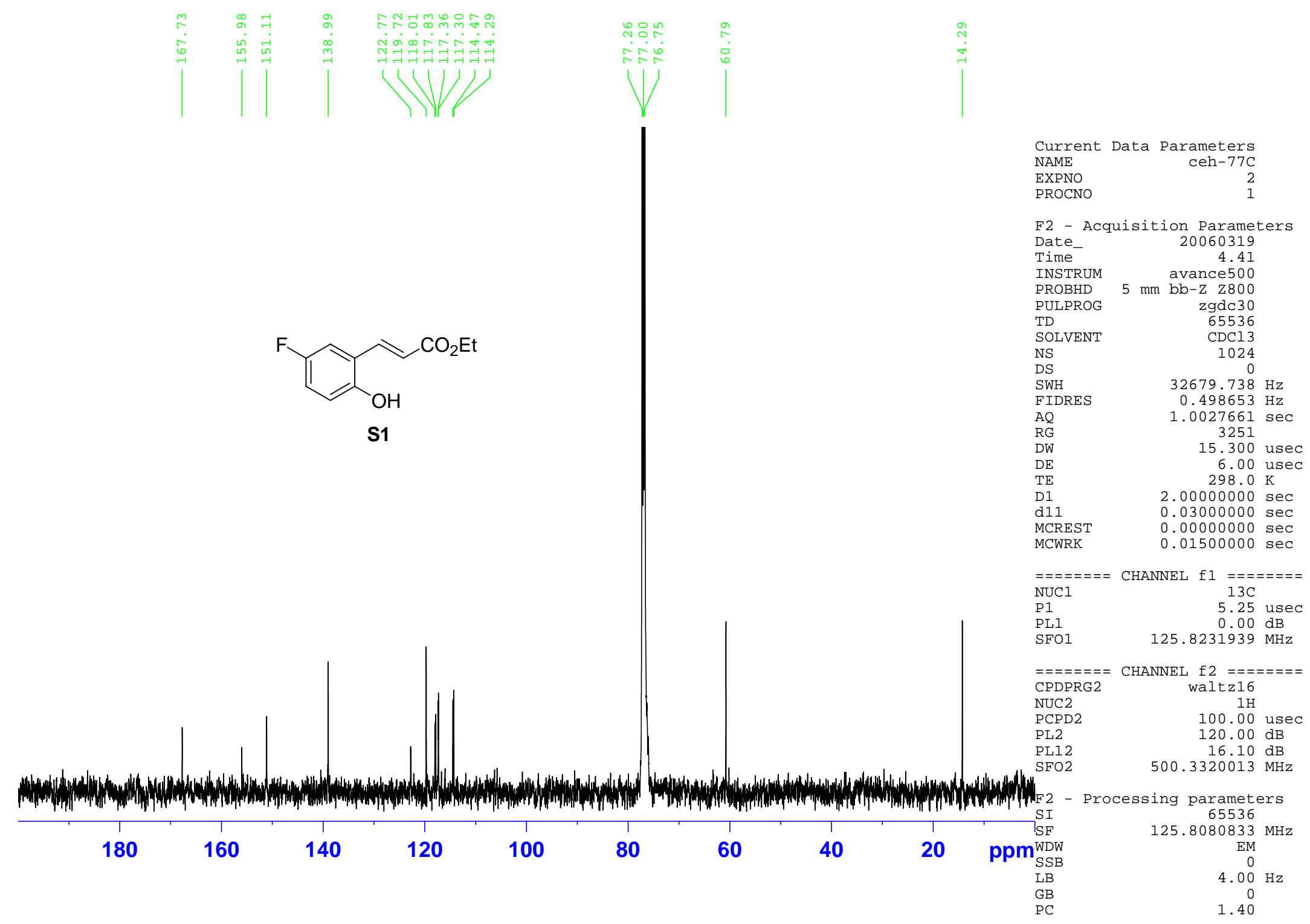




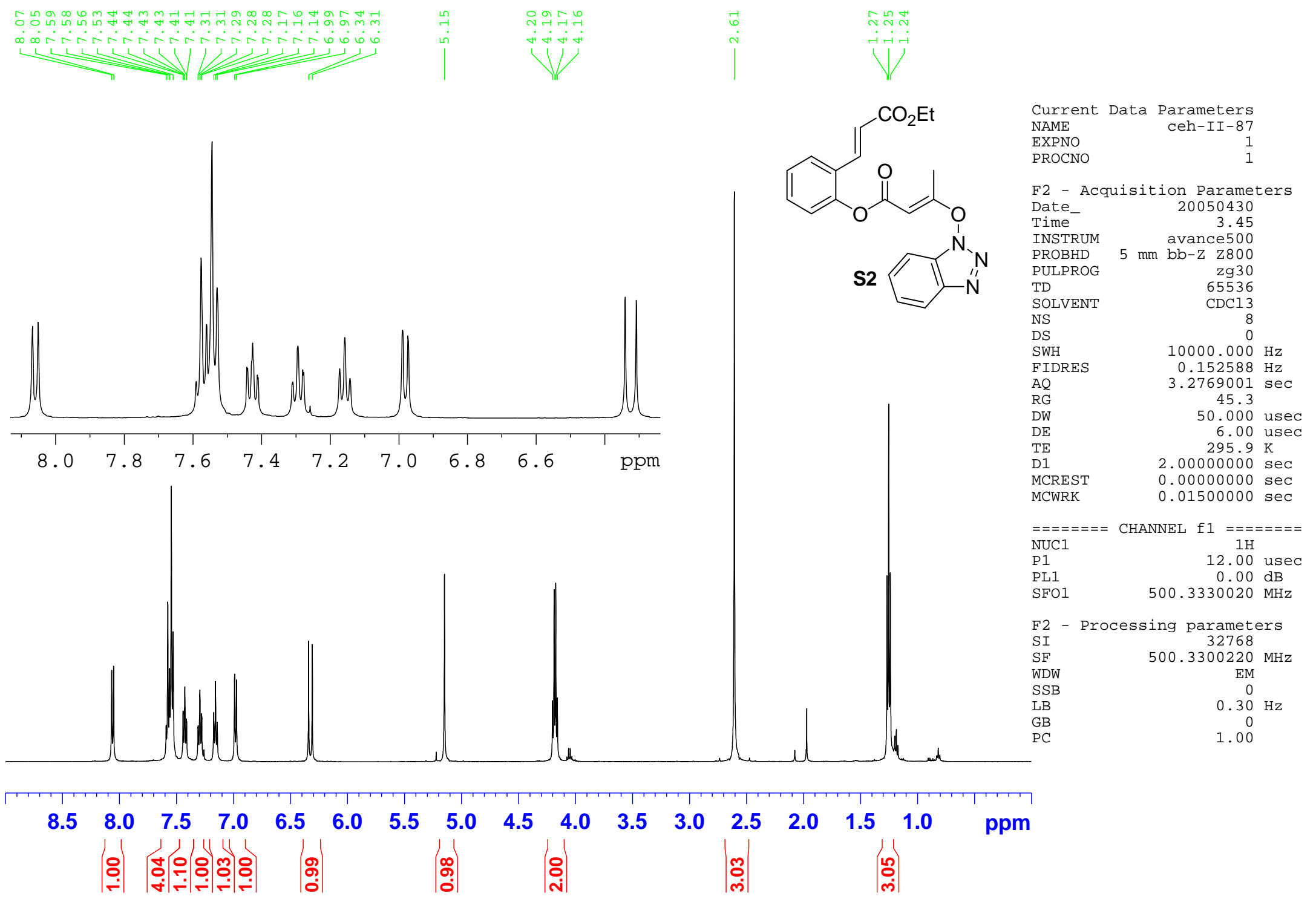




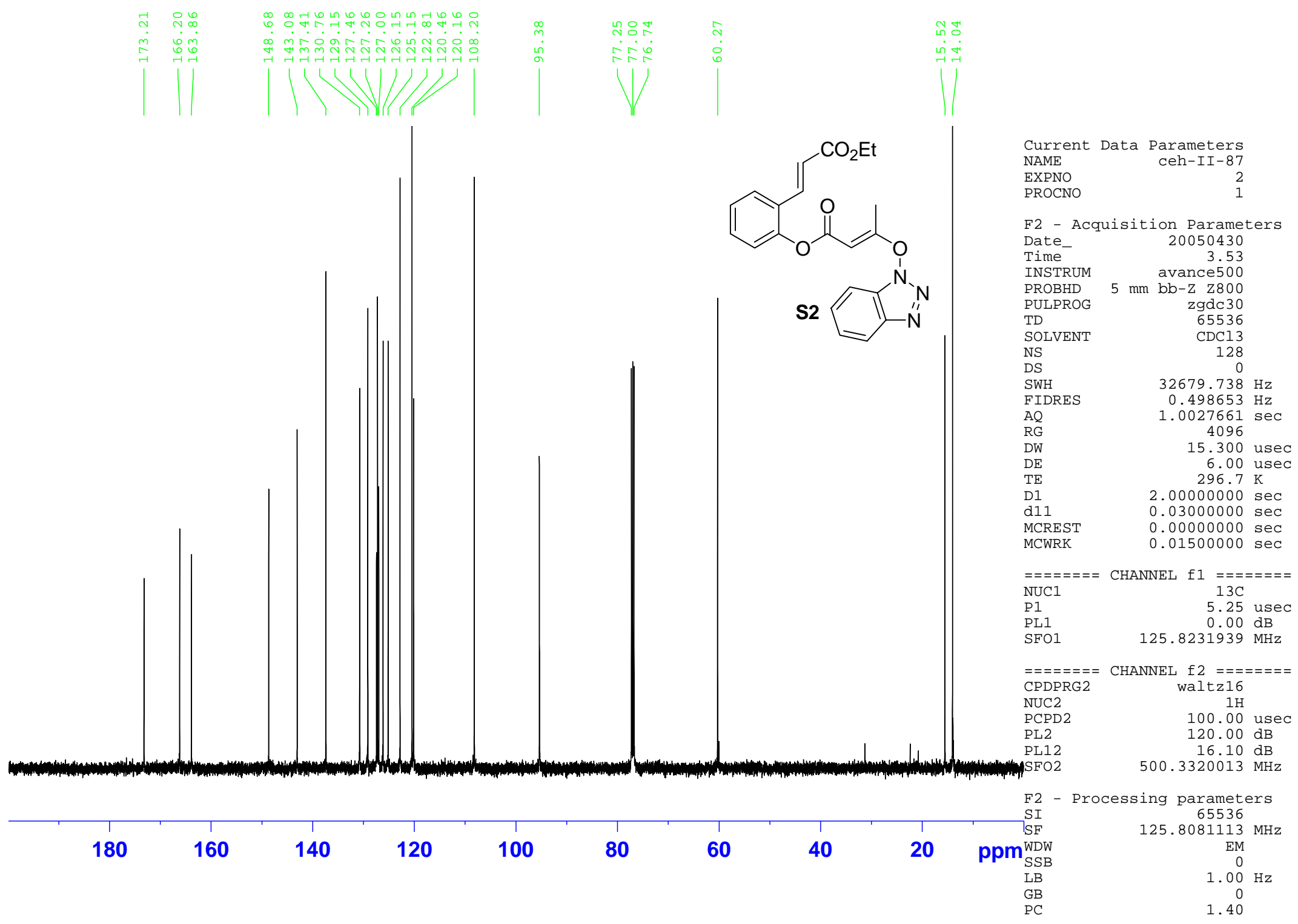



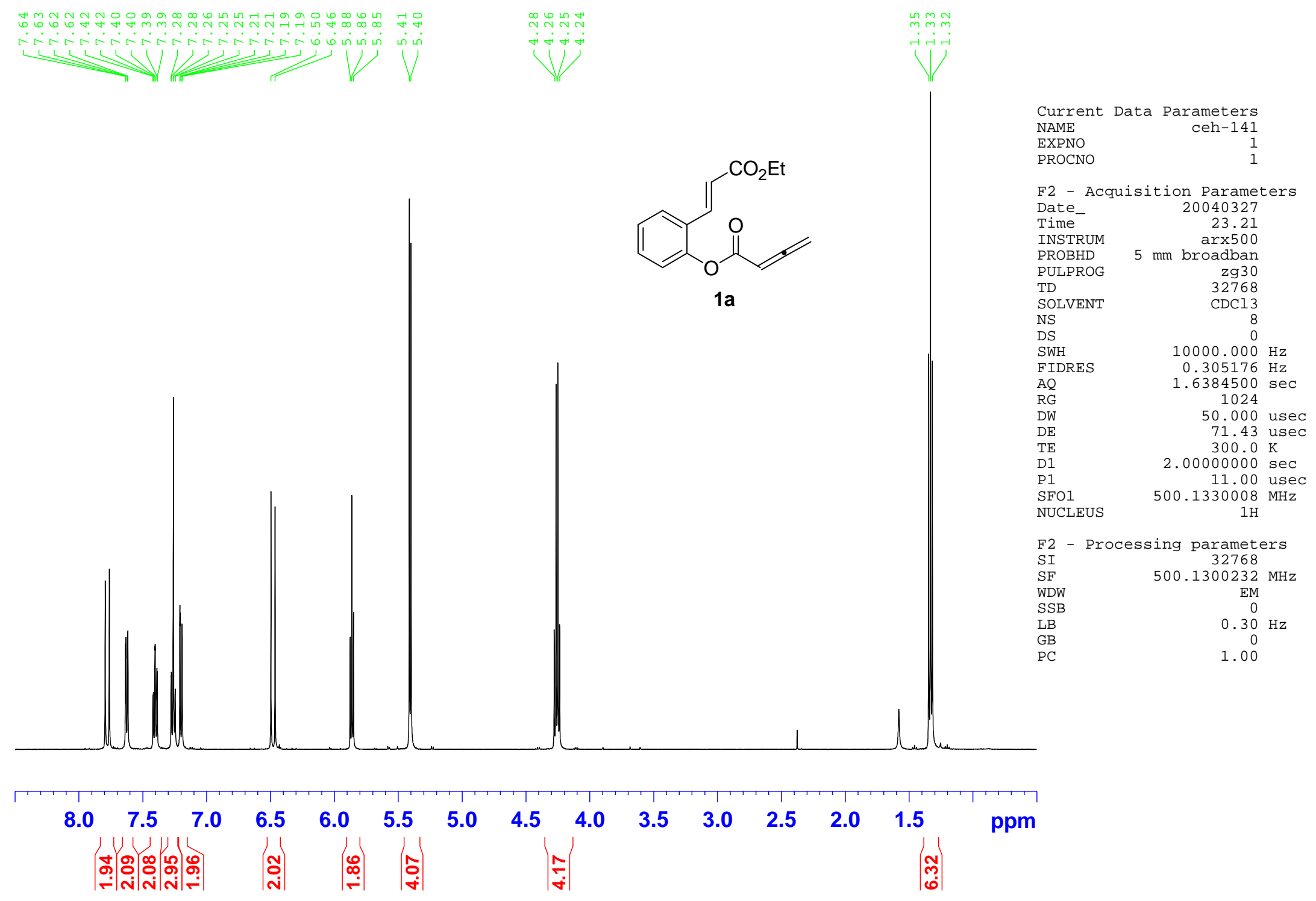

2 - Acquisition Parameter

(n)

FIDRES

1

NUCLEUS

$500.1300232 \mathrm{MHz}$

$0.30 \mathrm{~Hz}$
0
1.00 

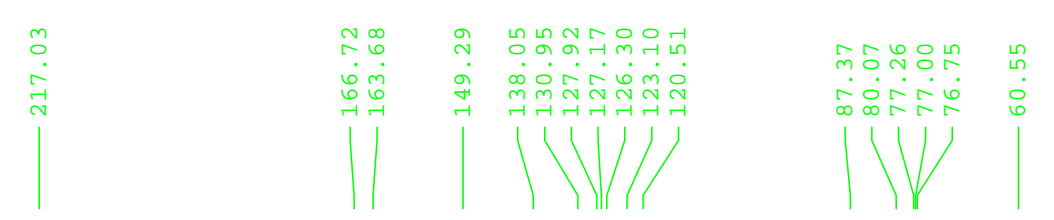

Current Data Parameters

NAME

PROCNO

ceh-141 1

F2 - Acquisition Parameters

Date

20040328

INSTRUM

PROBHD

PULPROG

$\operatorname{ar} \times 500$

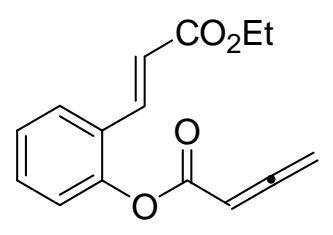

$1 a$
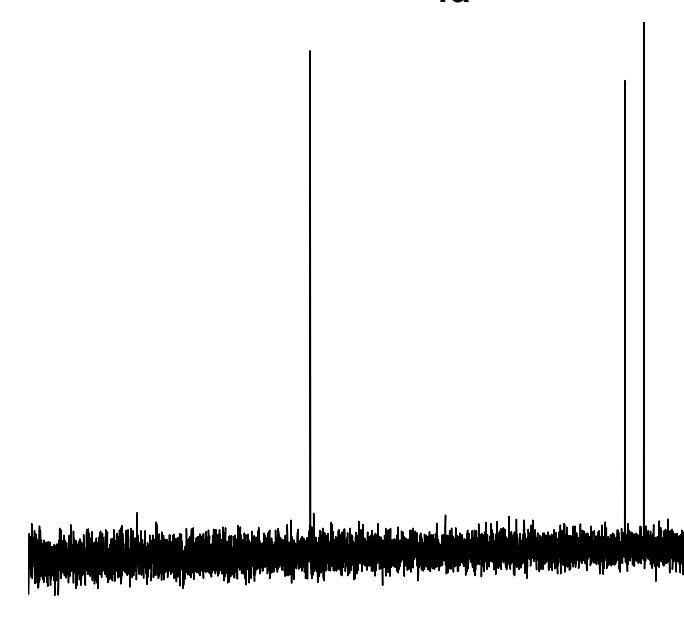

\section{T}

160

140

120

100

80

60

40

20

0 ppm 


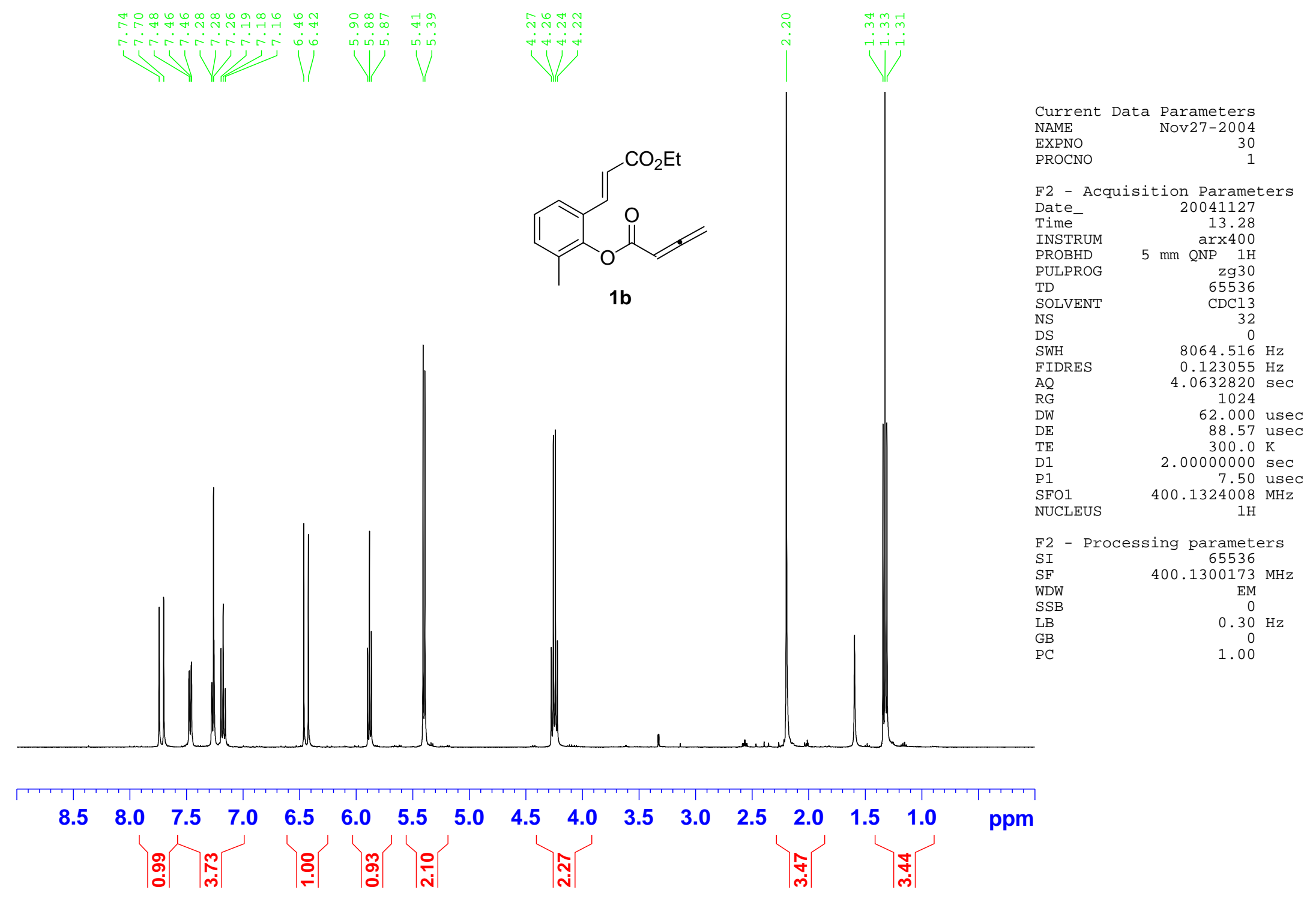




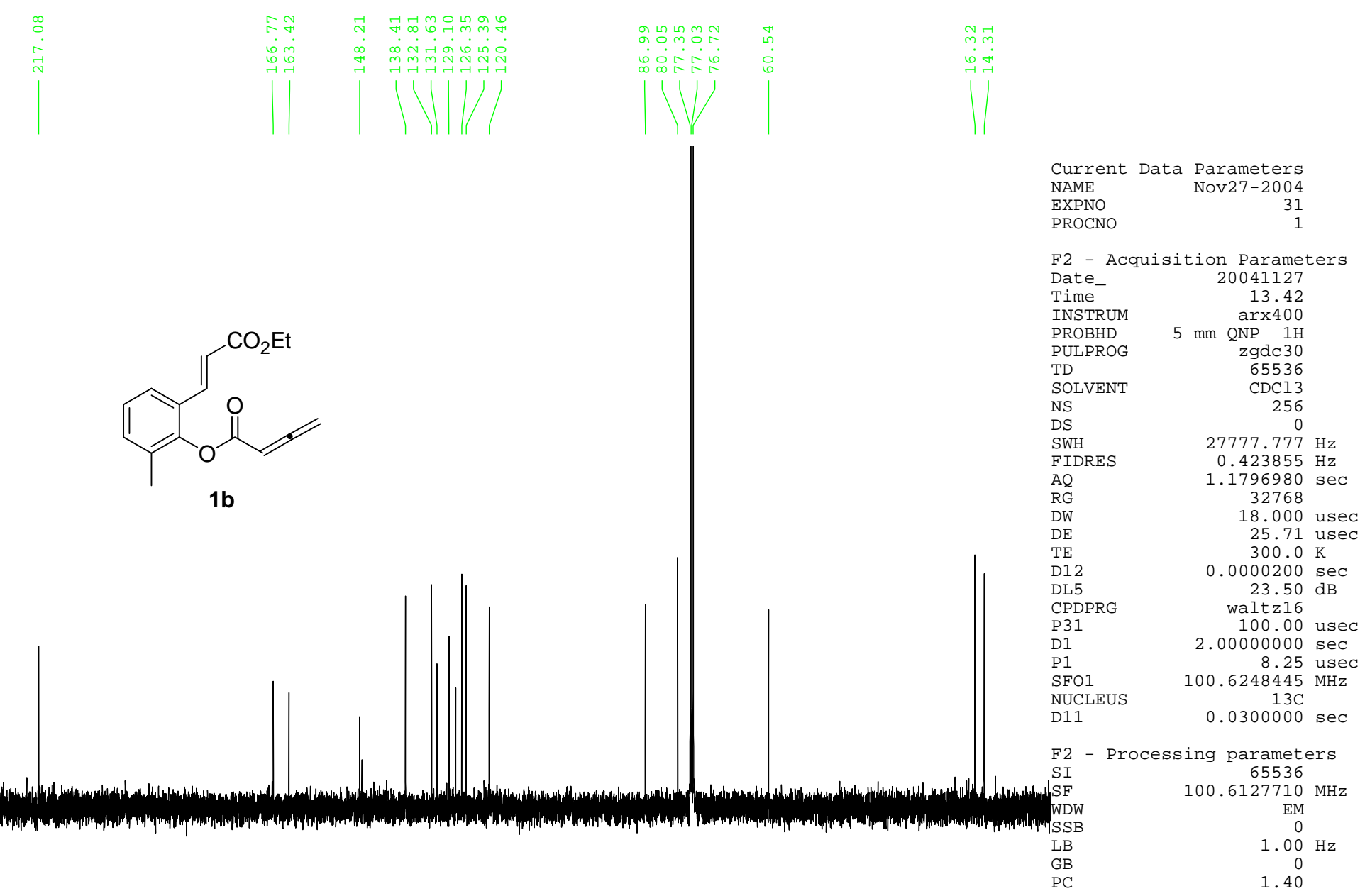

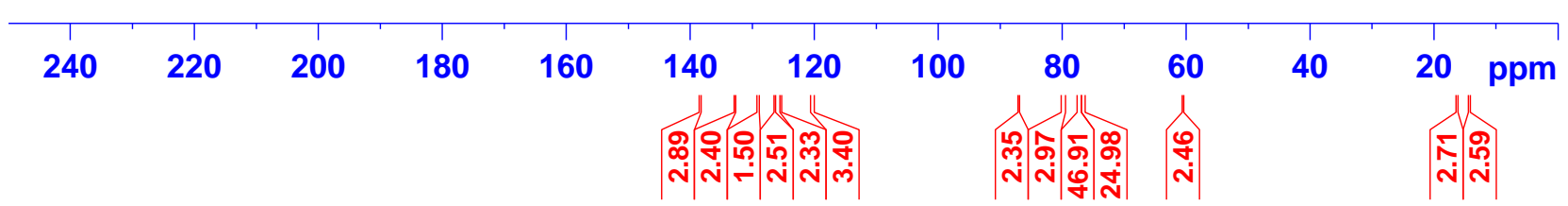




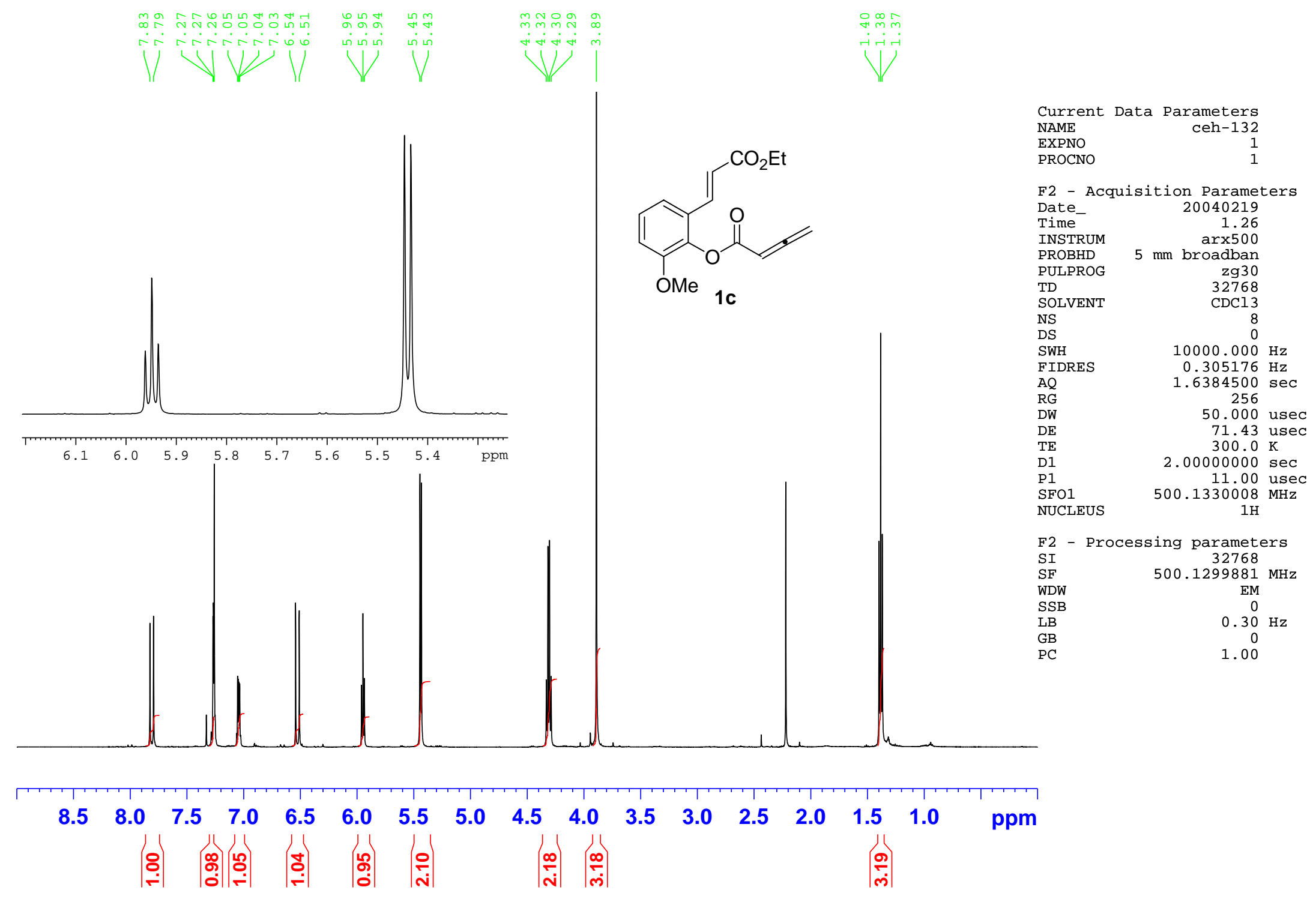



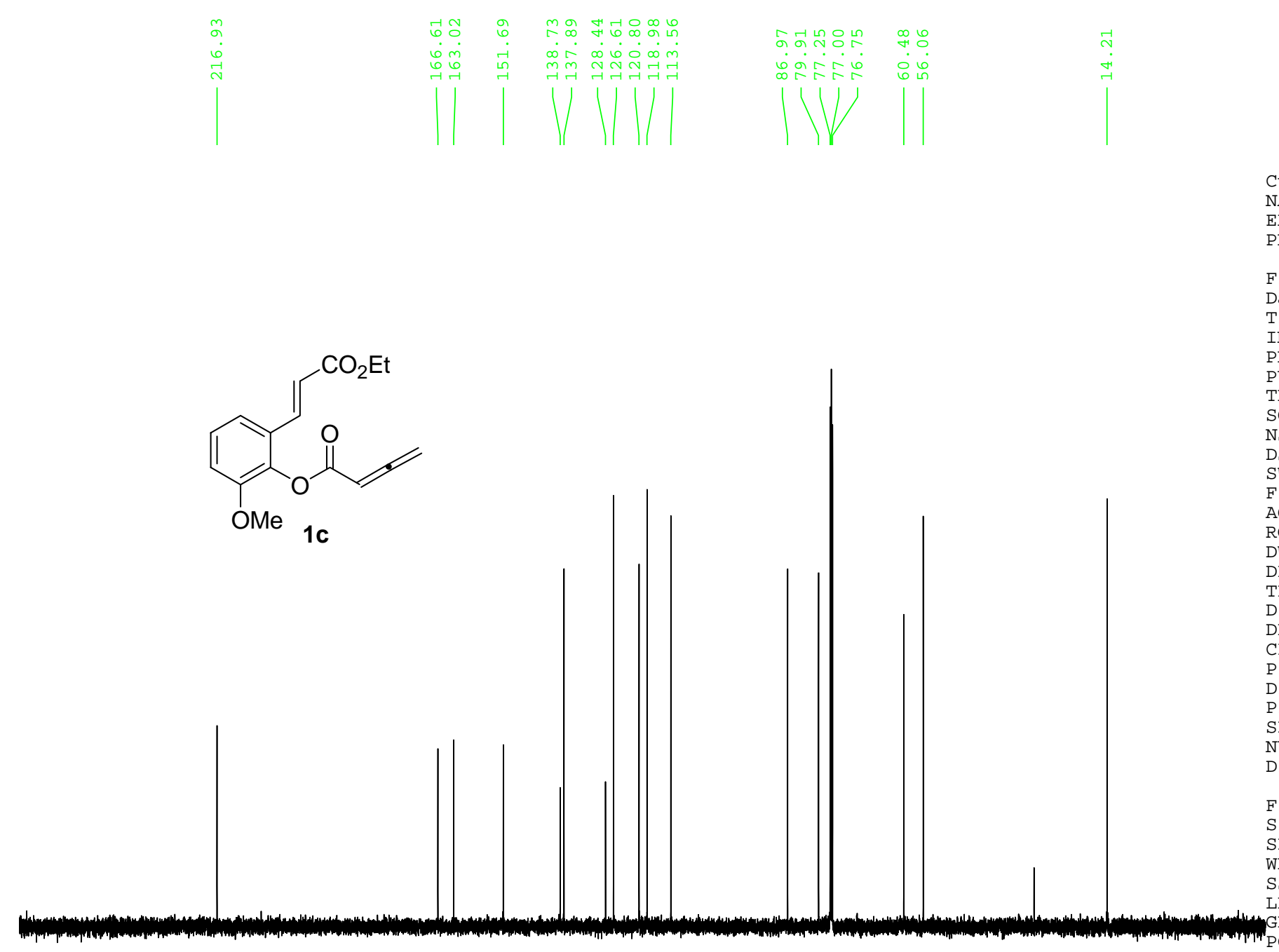

Current Data Parameters

NAME $\quad$ ceh-132

PROCNO

F2 - Acquisition Parameters

$\begin{array}{lr}\text { Date_ } & 20040227 \\ \text { Time } & 9.25\end{array}$

PULPROG $\quad$ zgdc30

TD 65536

SOLVENT CDCl3

DS

$\begin{array}{lr}\text { SWH } & 35714.285 \mathrm{~Hz} \\ \text { FIDRES } & 0.544957 \mathrm{~Hz}\end{array}$

AQ $\quad 0.9175540 \mathrm{sec}$

$\begin{array}{lr}\text { RG } & 45500 \\ \text { DW } & 14.000 \text { usec }\end{array}$

DE 20.0० usec

TE $300.0 \mathrm{~K}$

$\begin{array}{rr}\text { D12 } & 0.0000200 \mathrm{sec} \\ \text { DL5 } & 17.70 \mathrm{~dB}\end{array}$

DL5

CPDPRG

P31

D1
P1
SF01

waltz16

100.00 usec

NUCLEUS (

$125.7728999 \mathrm{MHz}$

D11 $\begin{array}{r}13 \mathrm{C} \\ \mathrm{Nec}\end{array}$

F2 - Processing parameters

SI 32768

SF $125.7578030 \mathrm{MHz}$

WDW

SSB

$\mathrm{EM}$
$\ominus$
$1.00 \mathrm{~Hz}$
$\odot$
1.40

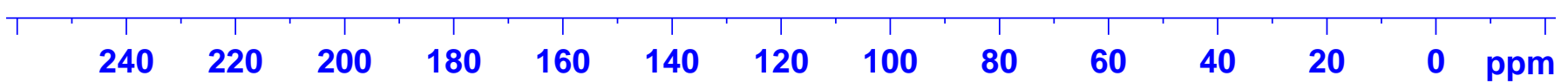




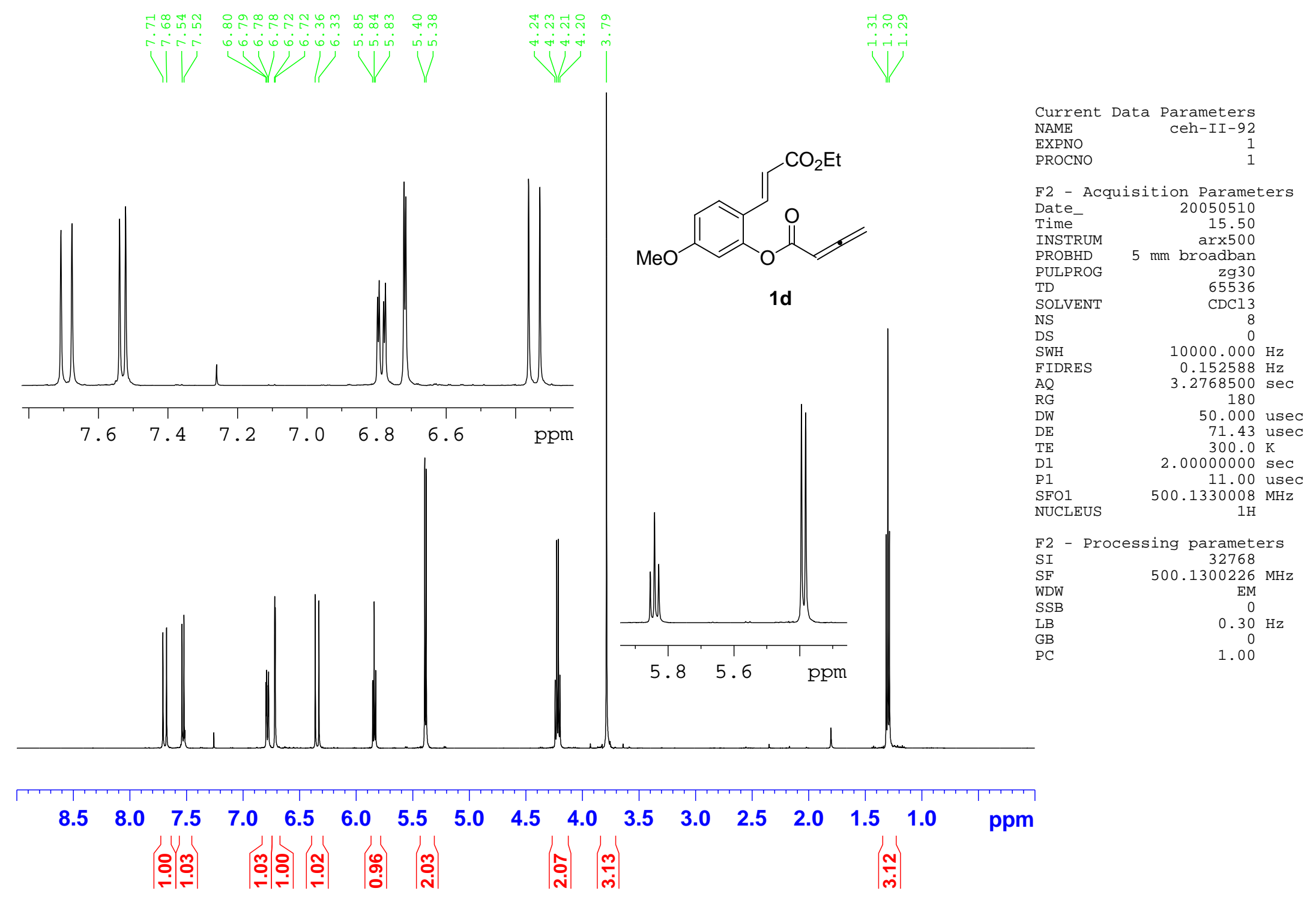




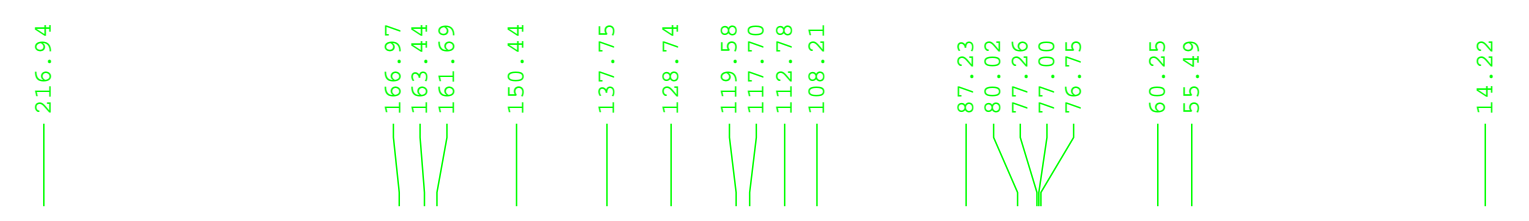

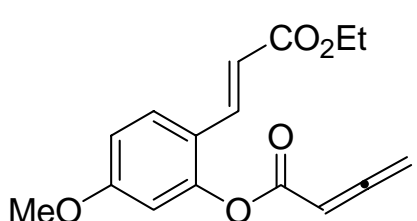

1d

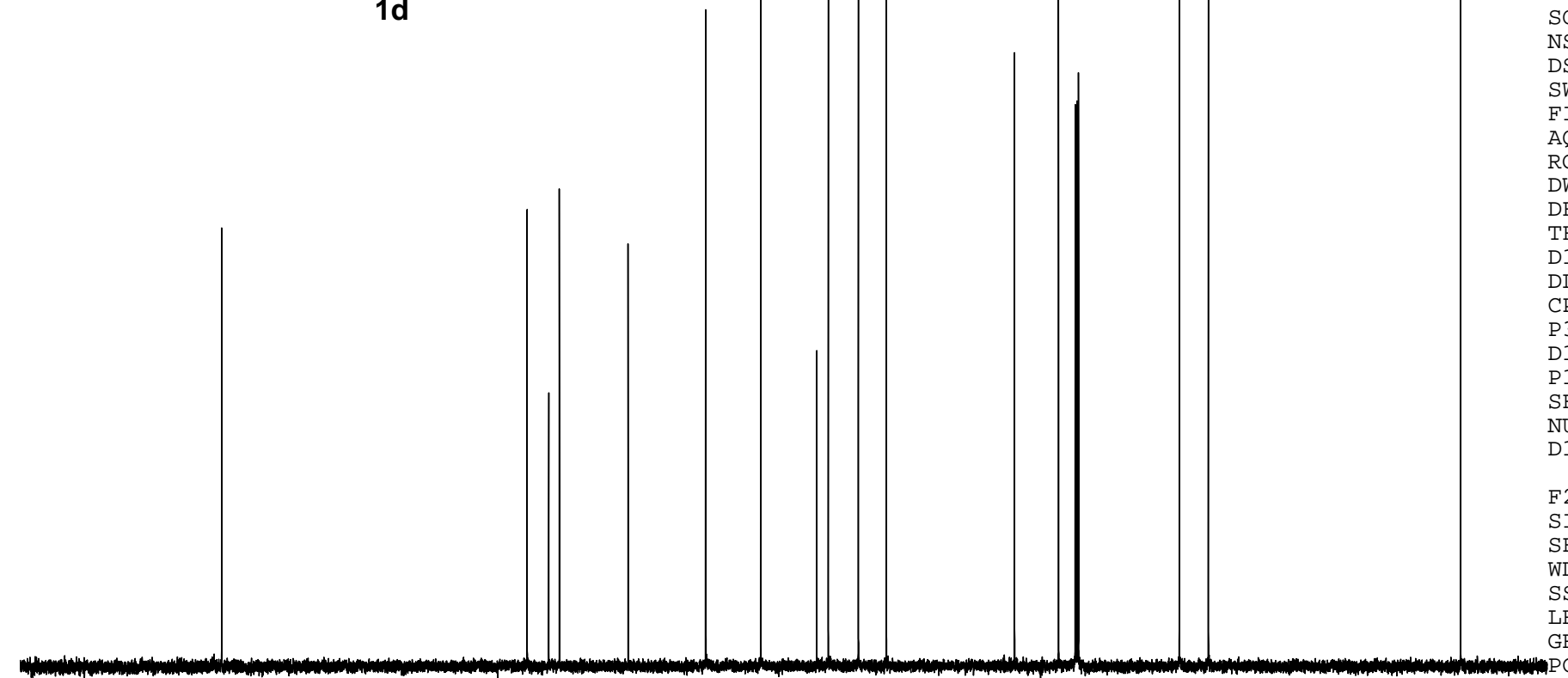

Current Data Parameters NAME ceh-II-92

PROCNO 2
1

F2 - Acquisition Parameters

$\begin{array}{lr}\text { Date_- } & 20050510 \\ \text { Time } & 15.57\end{array}$

$5 \mathrm{~mm}$ arx $\operatorname{ar} 50$

PULPROG 5

65536

SOLVENT CDCl3

NS
DS
SWH

$35714.285 \mathrm{~Hz}$

FIDRES $\quad 0.544957 \mathrm{~Hz}$

AQ $\quad 0.9175540 \mathrm{sec}$

$\mathrm{RG}$

0.9175540
16384

14.000 usec

$\begin{array}{lr}\text { DE } & 20.00 \mathrm{usec} \\ \mathrm{TE} & 300.0 \mathrm{~K}\end{array}$

D12 $0.0000200 \mathrm{sec}$

DL5 $17.70 \mathrm{~dB}$

CPDPRG waltz16

P31 100.00 usec

D1 2.00000000 sec

6.80 usec
$7728999 \mathrm{MHZ}$

SF01 125.7728999 MHz

NUCLEUS $13 \mathrm{C}$

D11 $0.0300000 \mathrm{sec}$

F2 - Processing parameters

32768

SF $\quad 125.7578063 \mathrm{MHz}$

WDW

SSB

$G B$

$E M$
$\ominus$
$1.0 \ominus \mathrm{Hz}$
$\Theta$
$1.4 \Theta$

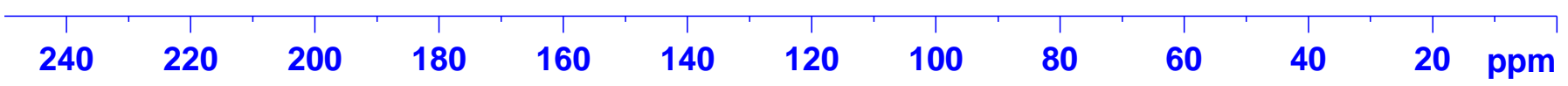




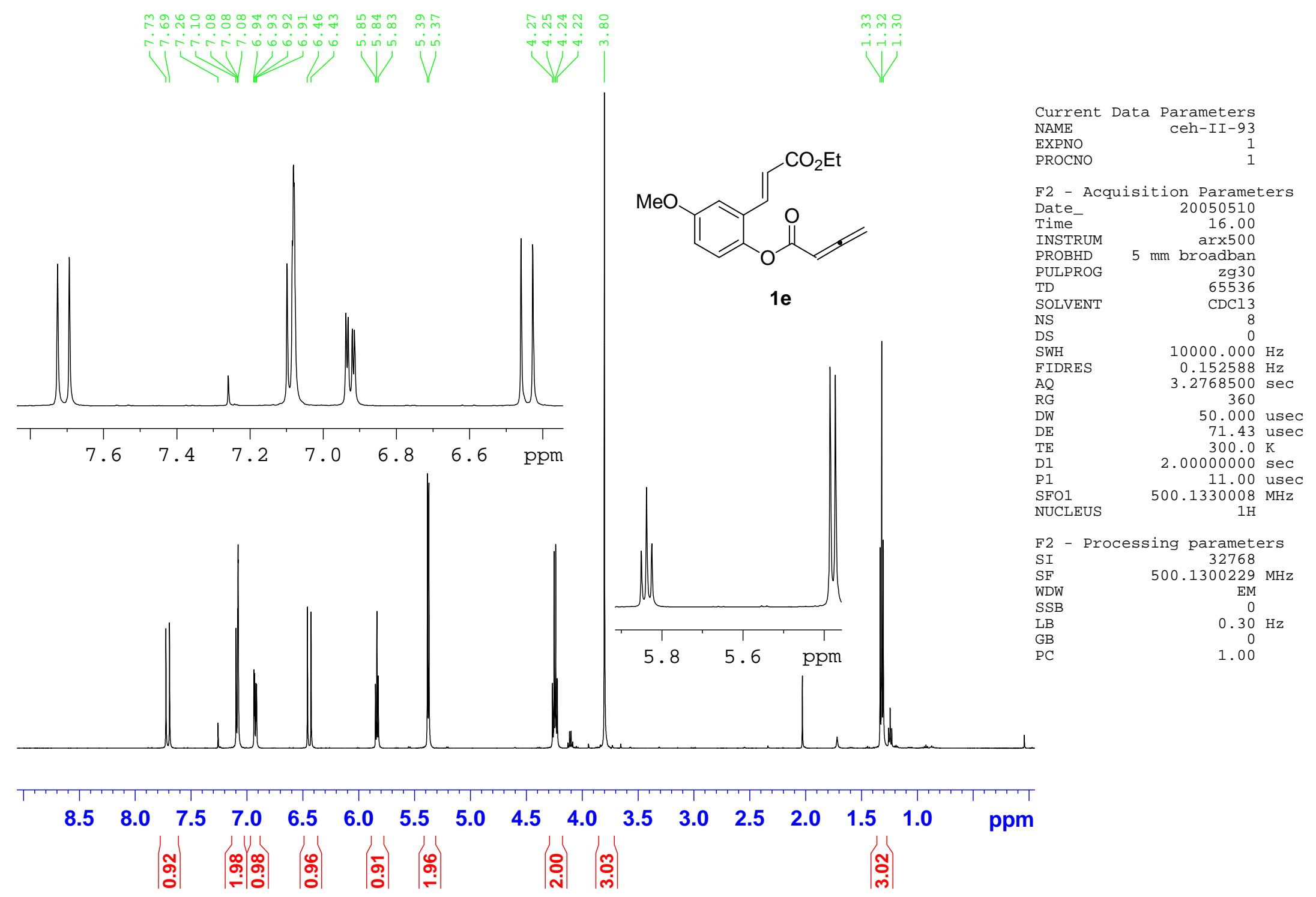



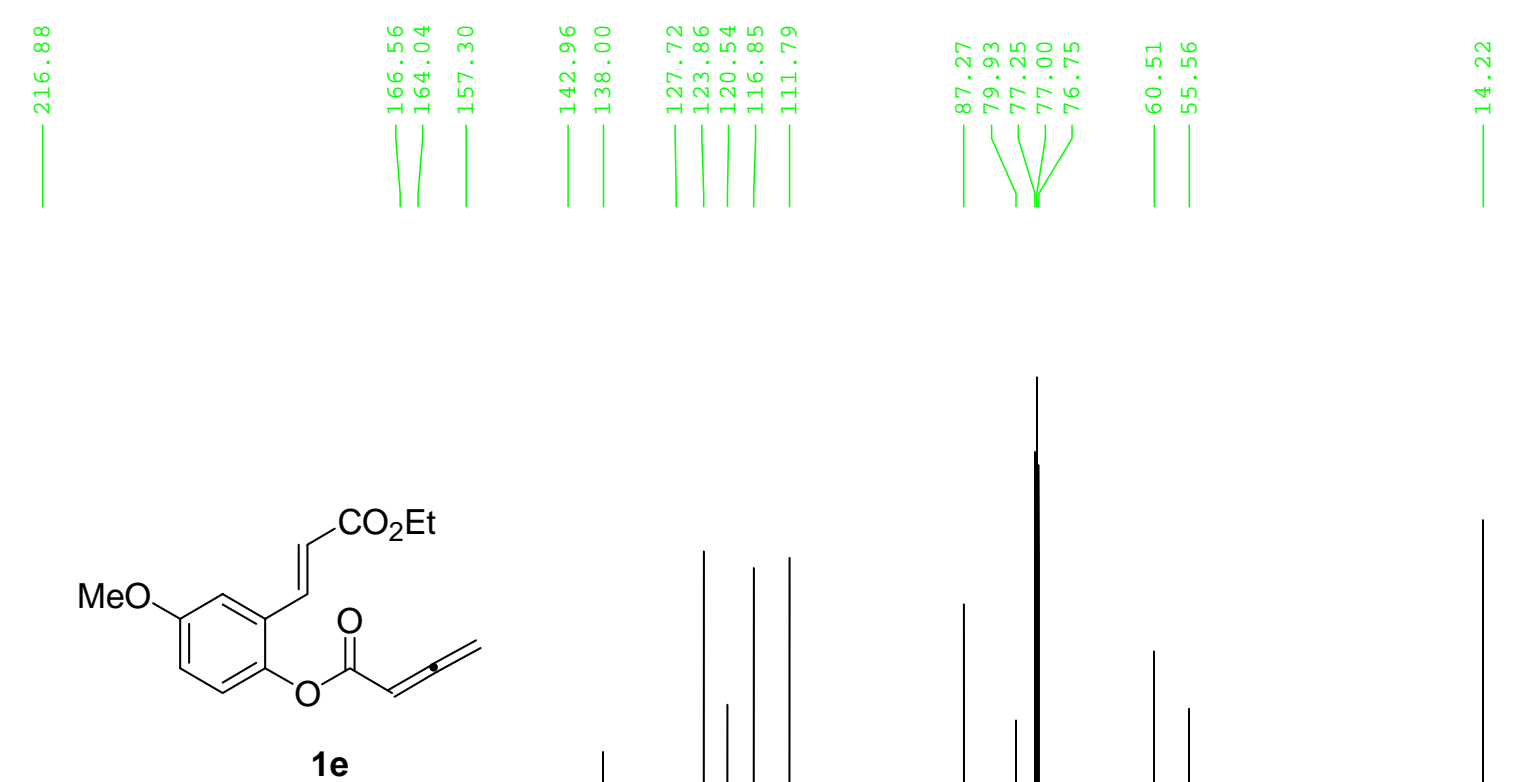

$1 e$

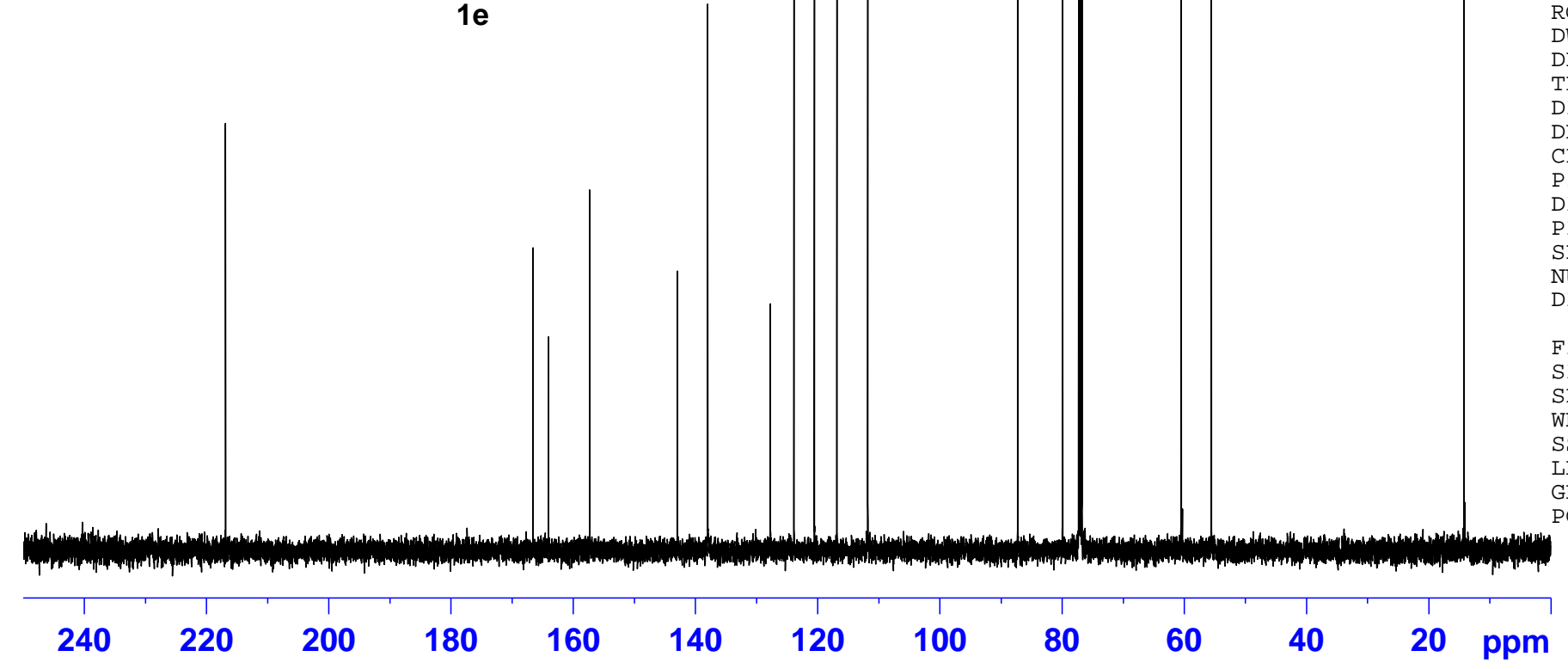

Current Data Parameters 


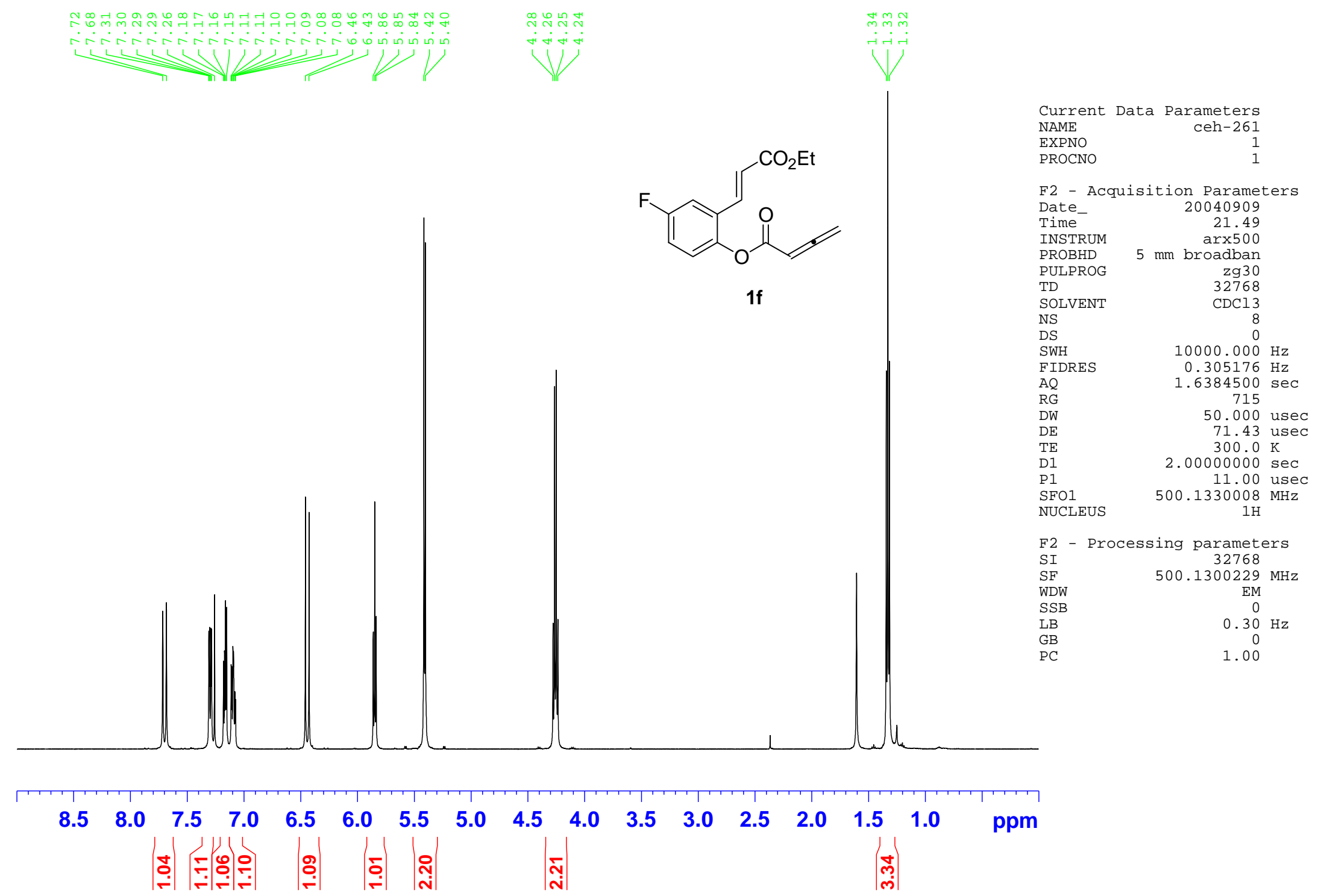




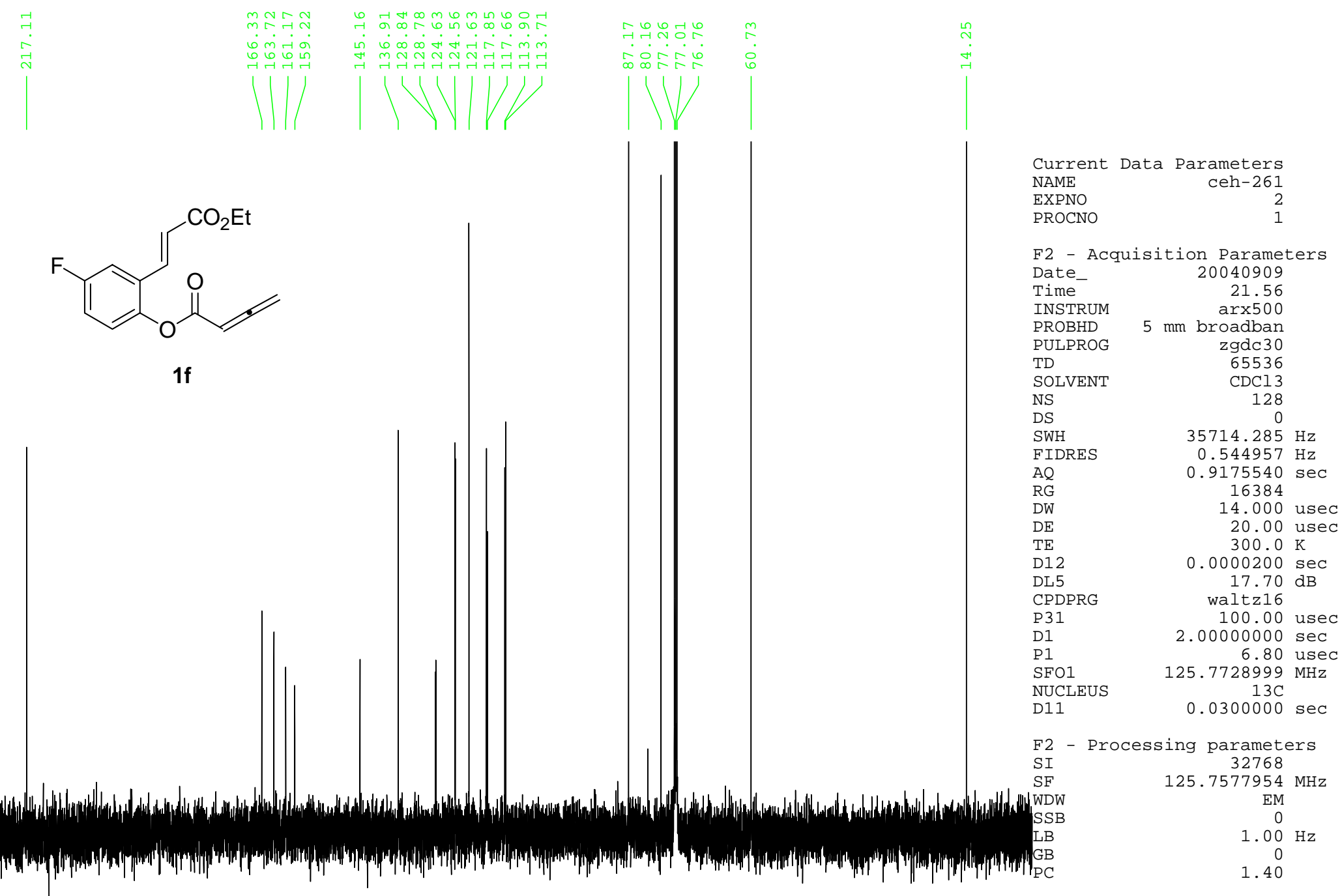

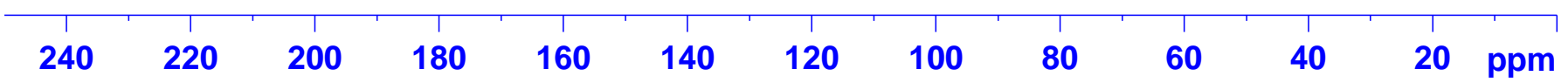




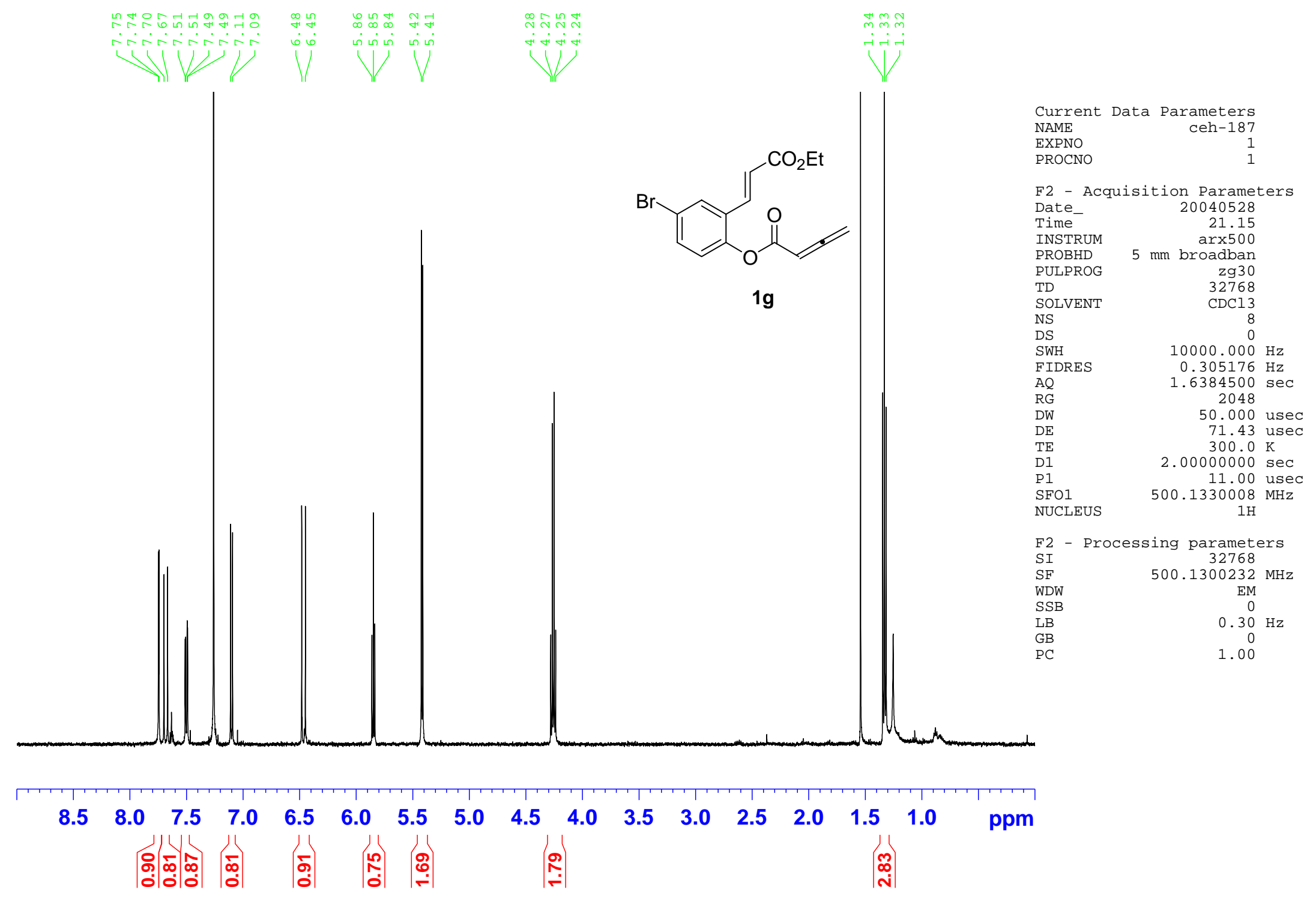




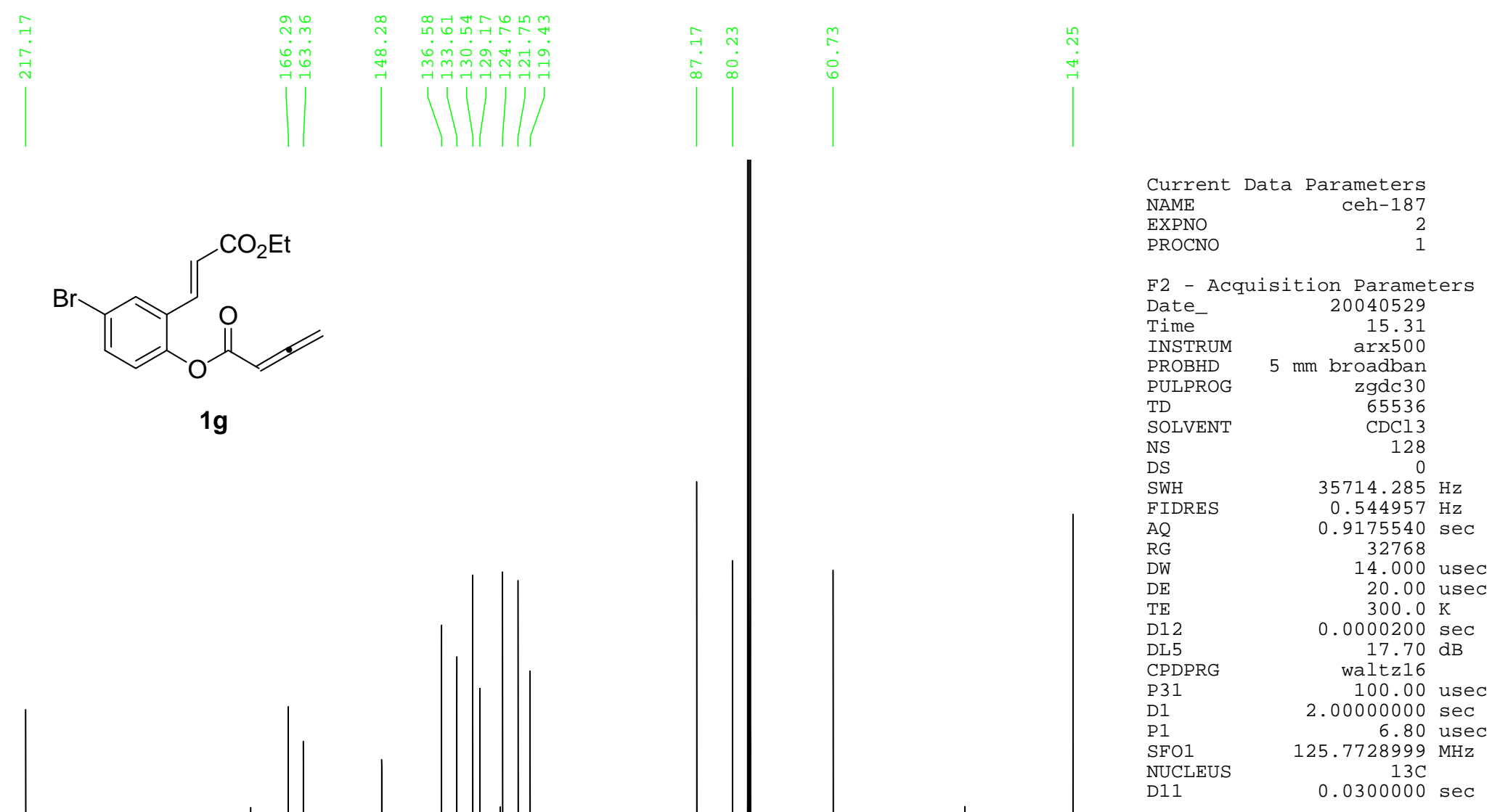

1 . Processing parameters

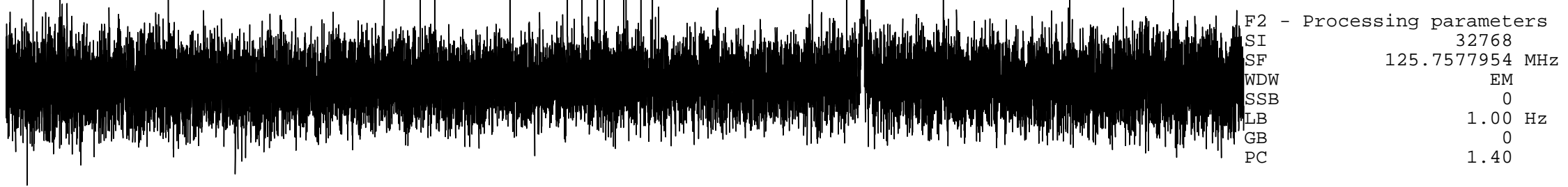

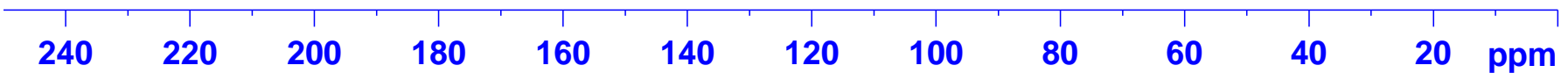




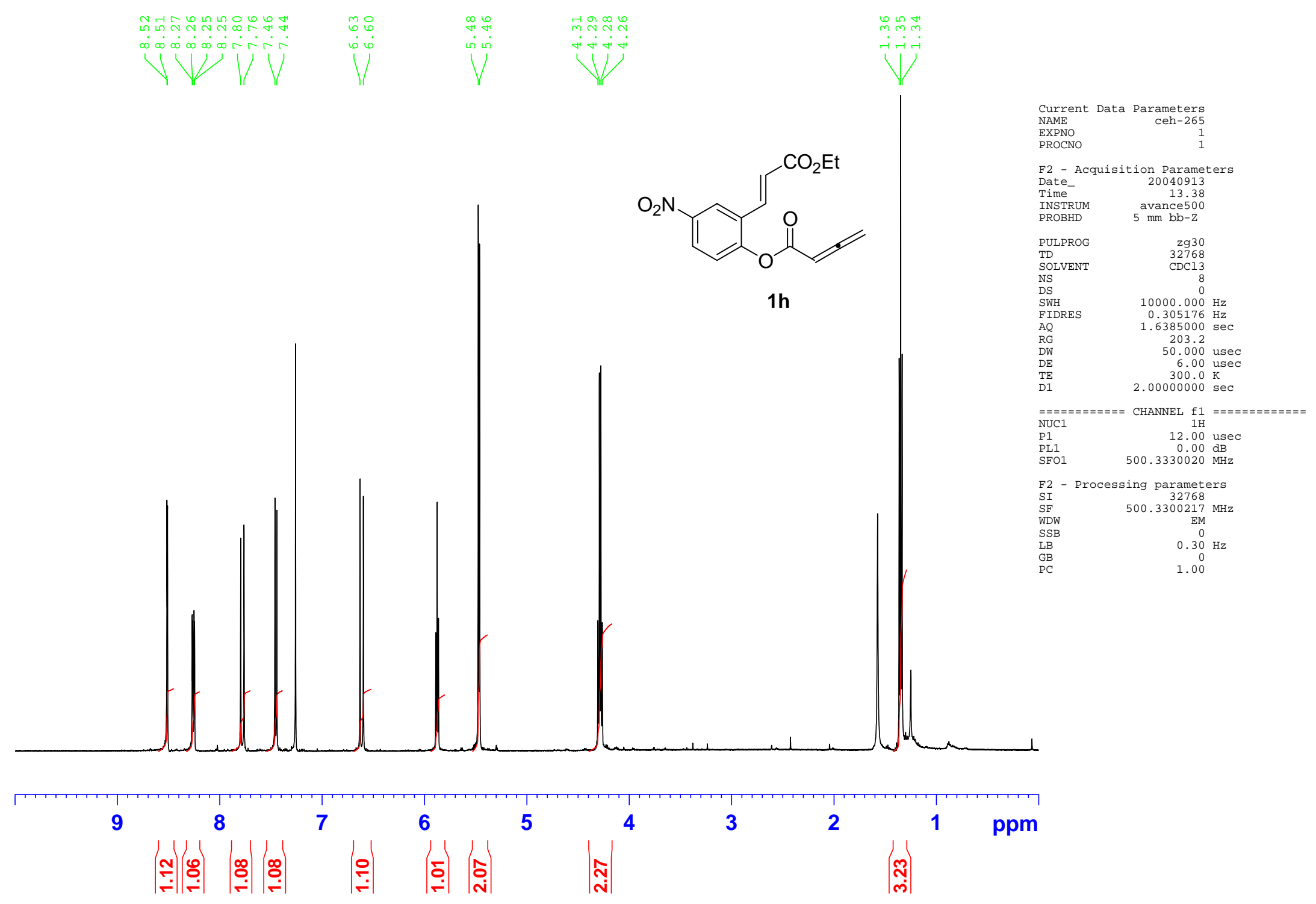



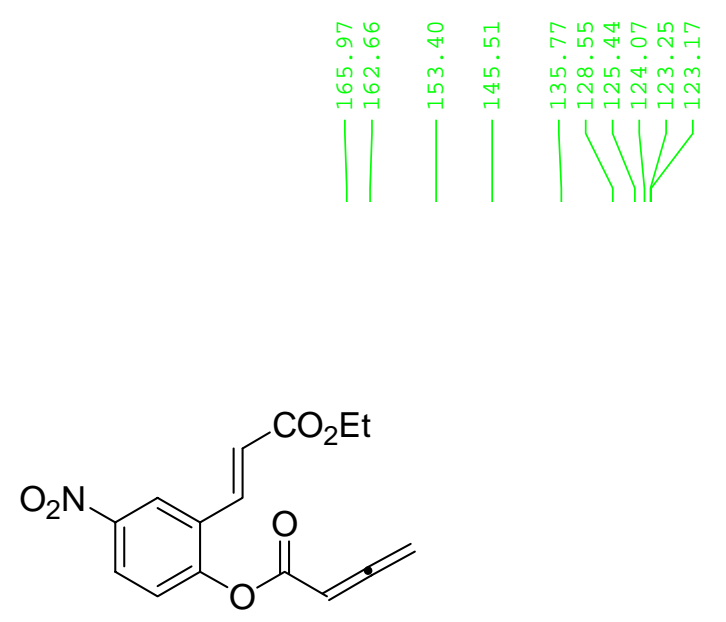

$1 h$

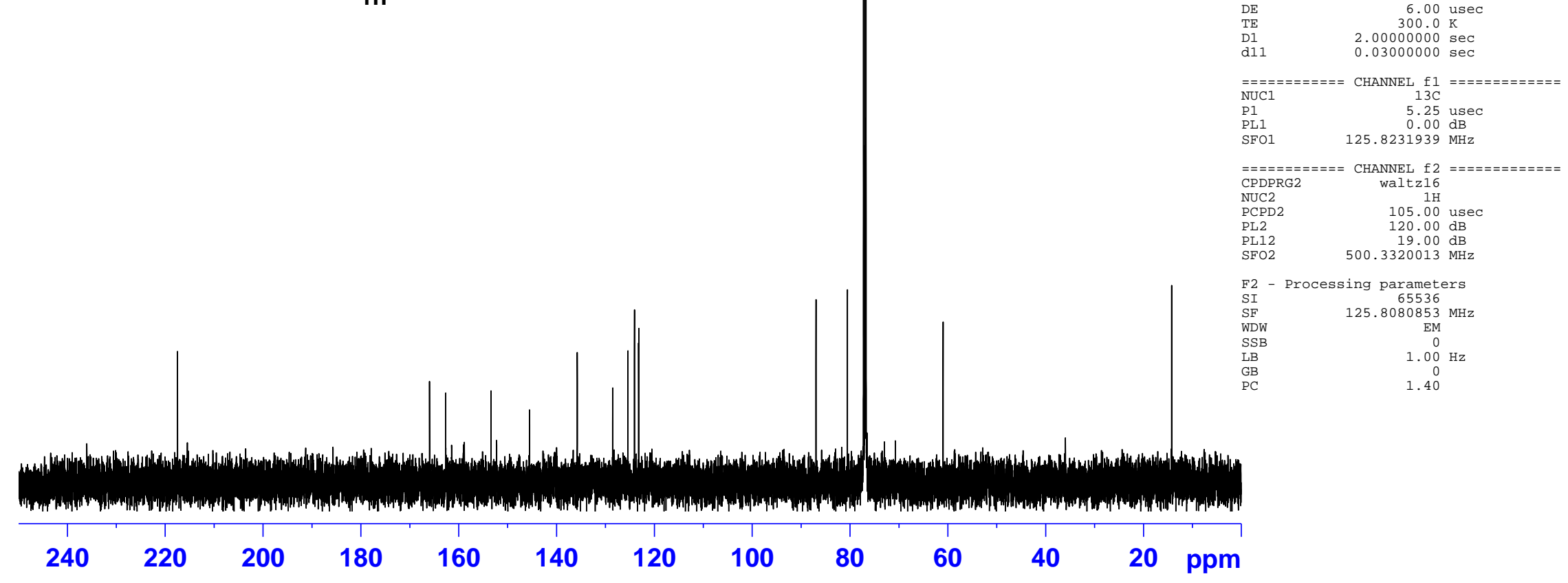

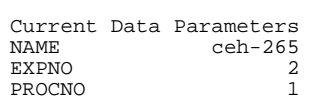

F2 - Acquisition Parameters

avance500
INSTRUM
PROBHD

PULPROG
Tgdc 30
65536

TD
SOLVENT

SOLVENT
NS
DS

$\begin{array}{lr}\text { SWH } & 32679.738 \mathrm{~Hz} \\ \text { FIDRES } & 0.498653 \mathrm{~Hz}\end{array}$

$\begin{array}{lr}\text { FIDRES } & 0.498653 \mathrm{~Hz} \\ \mathrm{AQ} & 1.0027661 \mathrm{sec}\end{array}$

$\begin{array}{lr}\text { AQ } & 4096 \\ \text { RG } & 15.300 \text { usec } \\ \text { DW } & .6 .00 \text {. }\end{array}$

$\begin{array}{lr}\text { DW } & 15.300 \text { usec } \\ \text { DE } & 6.00 \text { usec }\end{array}$

$\begin{array}{ll}\text { D1 } & 2.000000000 \mathrm{sec} \\ \text { d11 } & 0.03000000 \mathrm{sec}\end{array}$

$===========$
NUC1

$0.00 \mathrm{~dB}$

$=========$ CHANNEL $\mathrm{f} 2$

$\begin{array}{ll}\text { NUC2 } & 1 \mathrm{H} \\ \text { PCPD2 } & 105 .\end{array}$

$19.00 \mathrm{~dB}$
$\mathrm{P}$

$\begin{array}{lr}\text { SF } & 125.8080853 \\ \text { MHz }\end{array}$

0 $\begin{array}{lr}\text { Date } & 20040913 \\ \text { Time } & 13.45\end{array}$

F2 - Processing parameter 


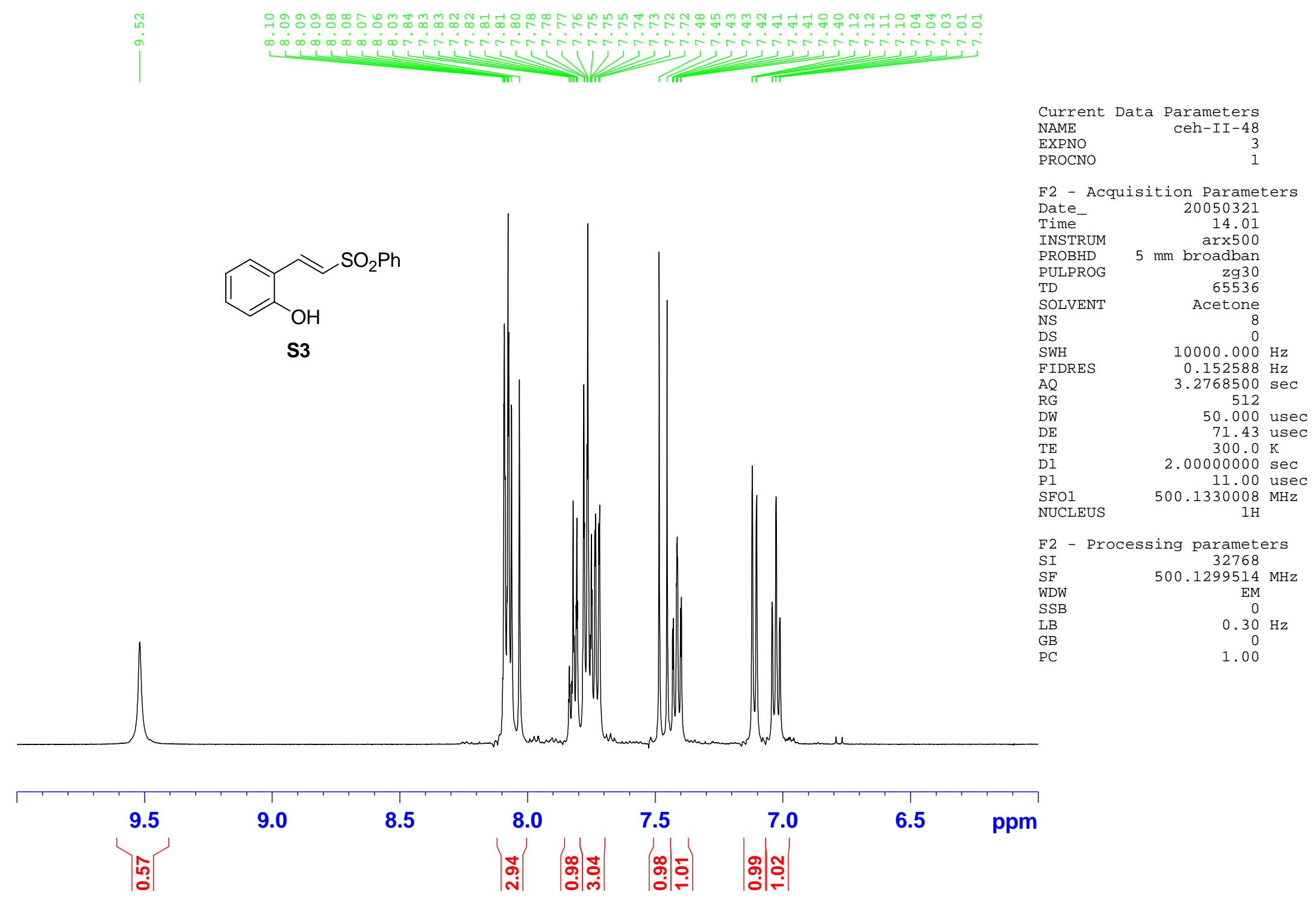




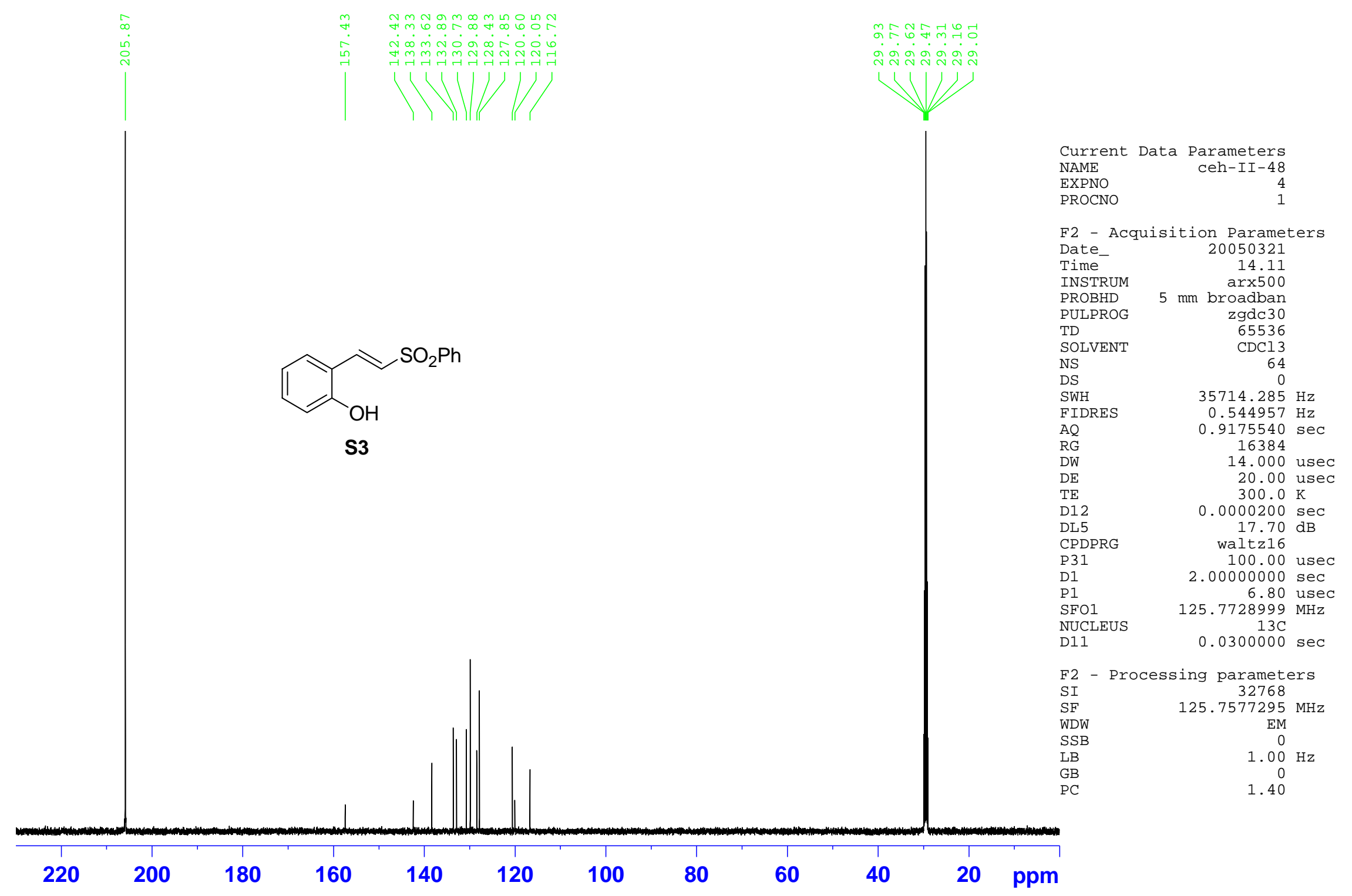




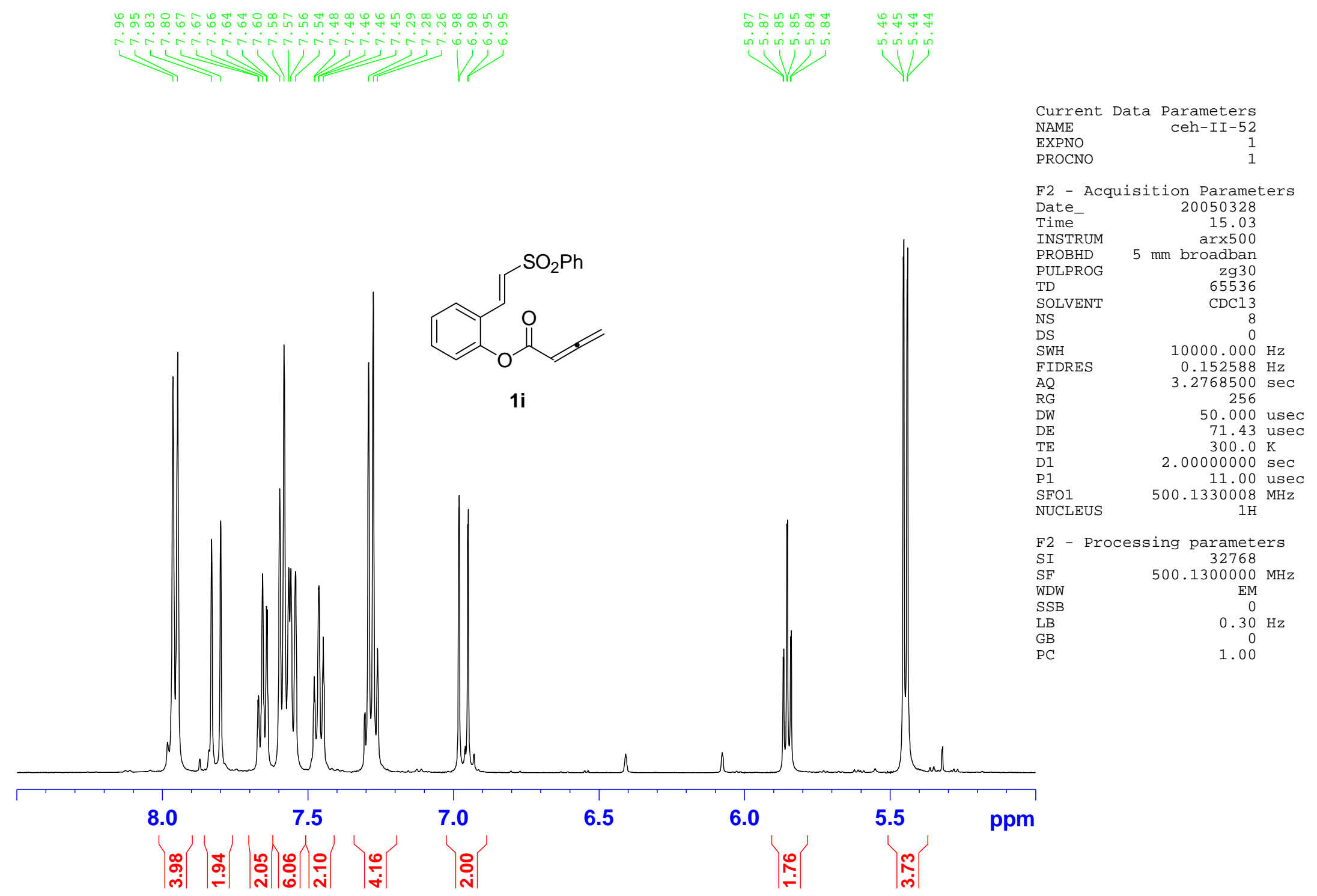




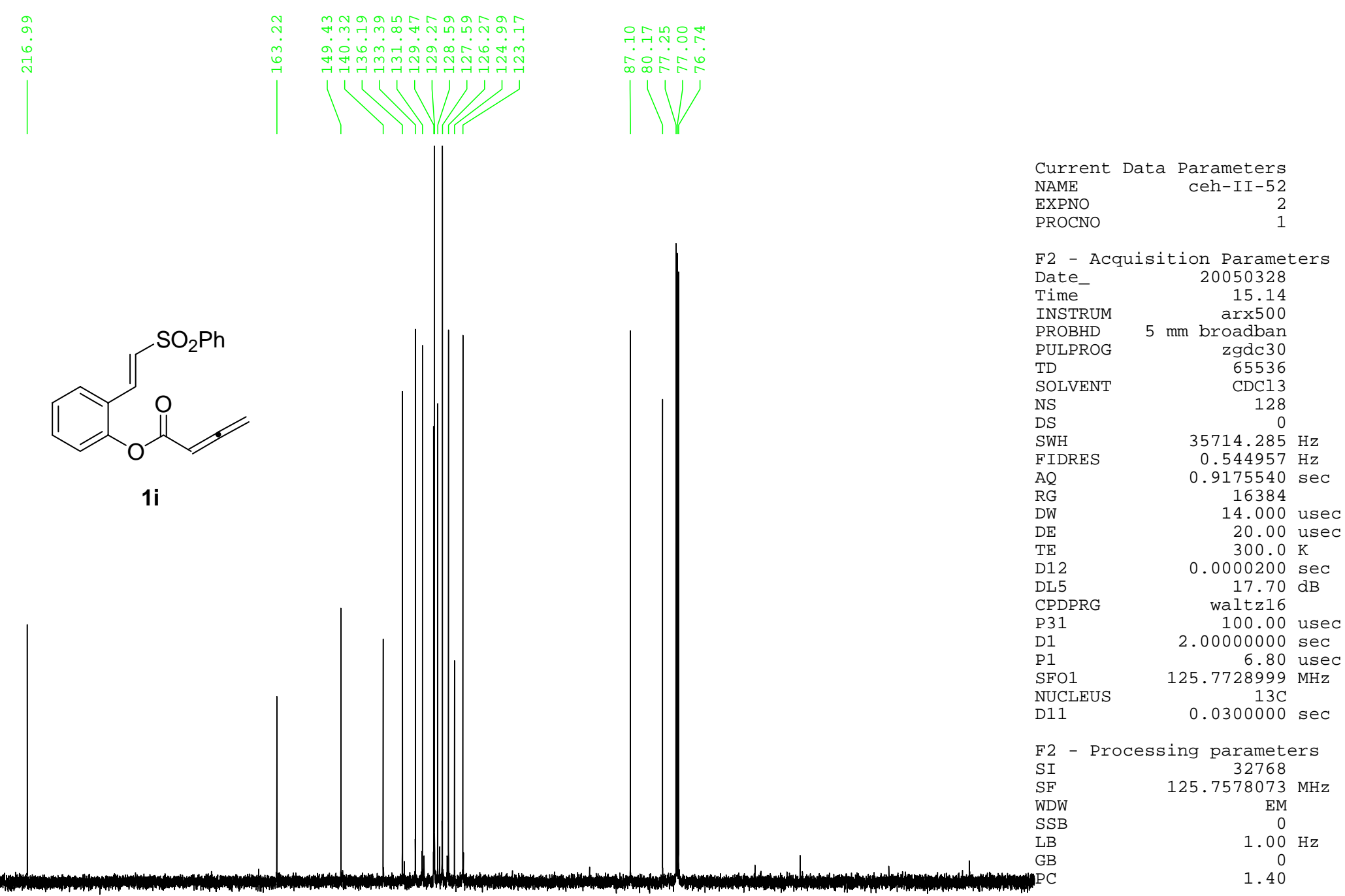

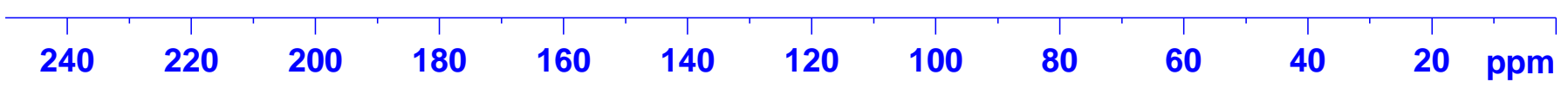




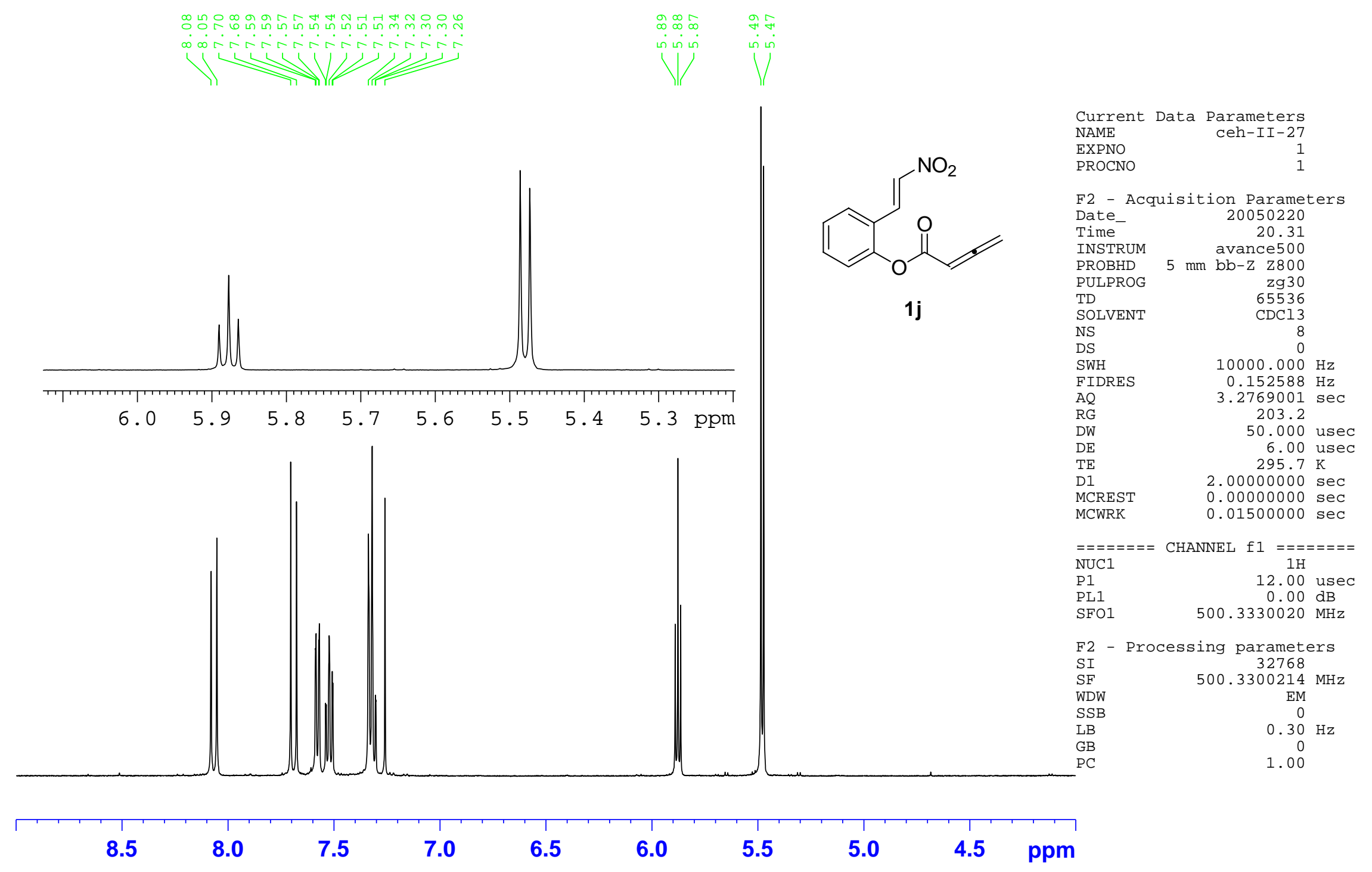




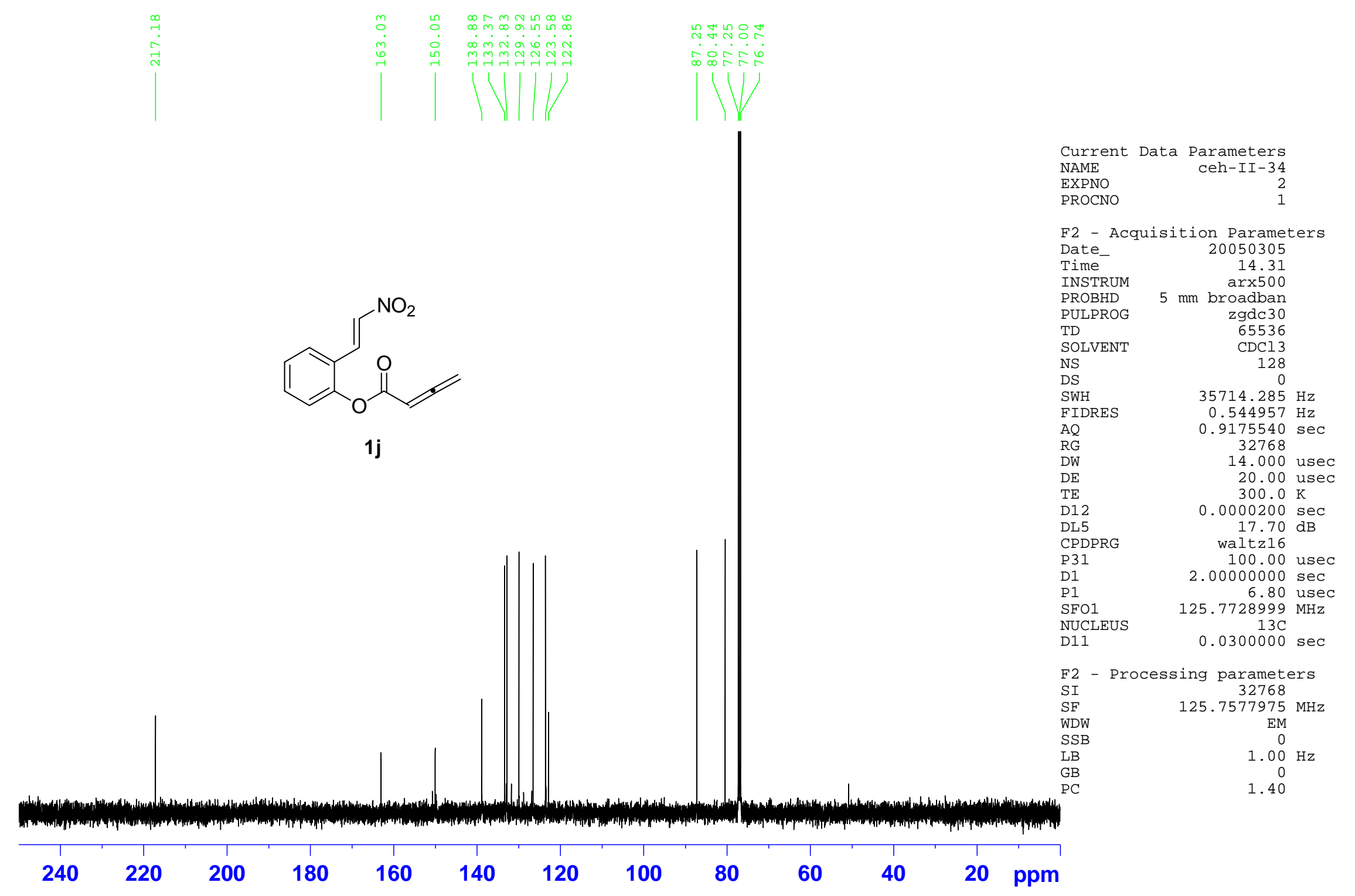




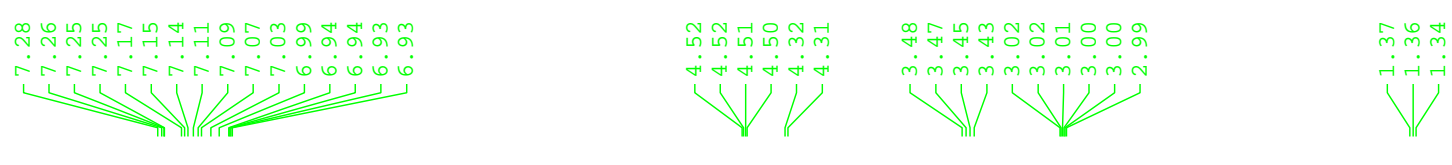

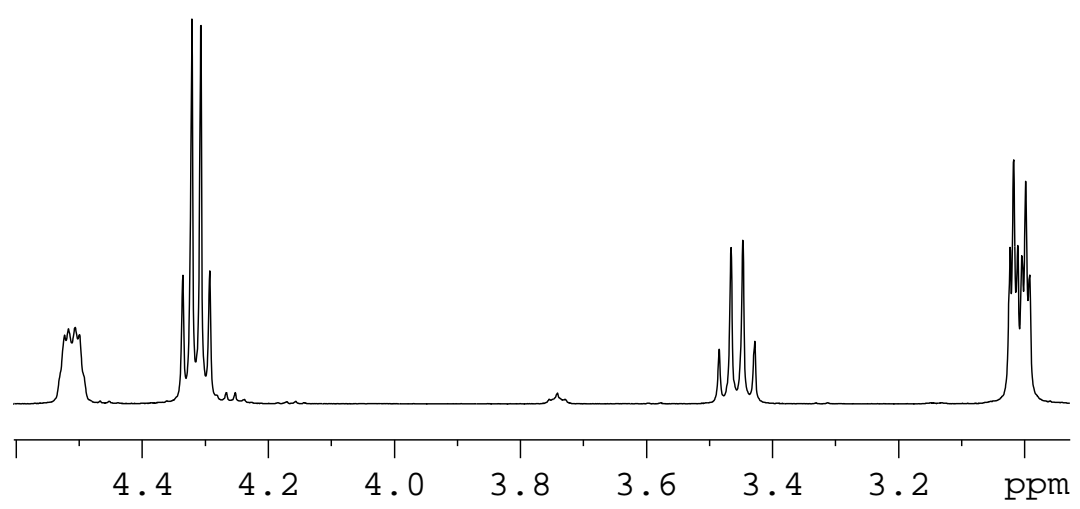

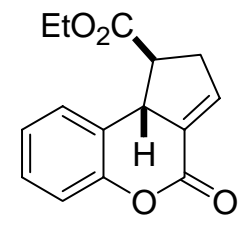

$2 a$

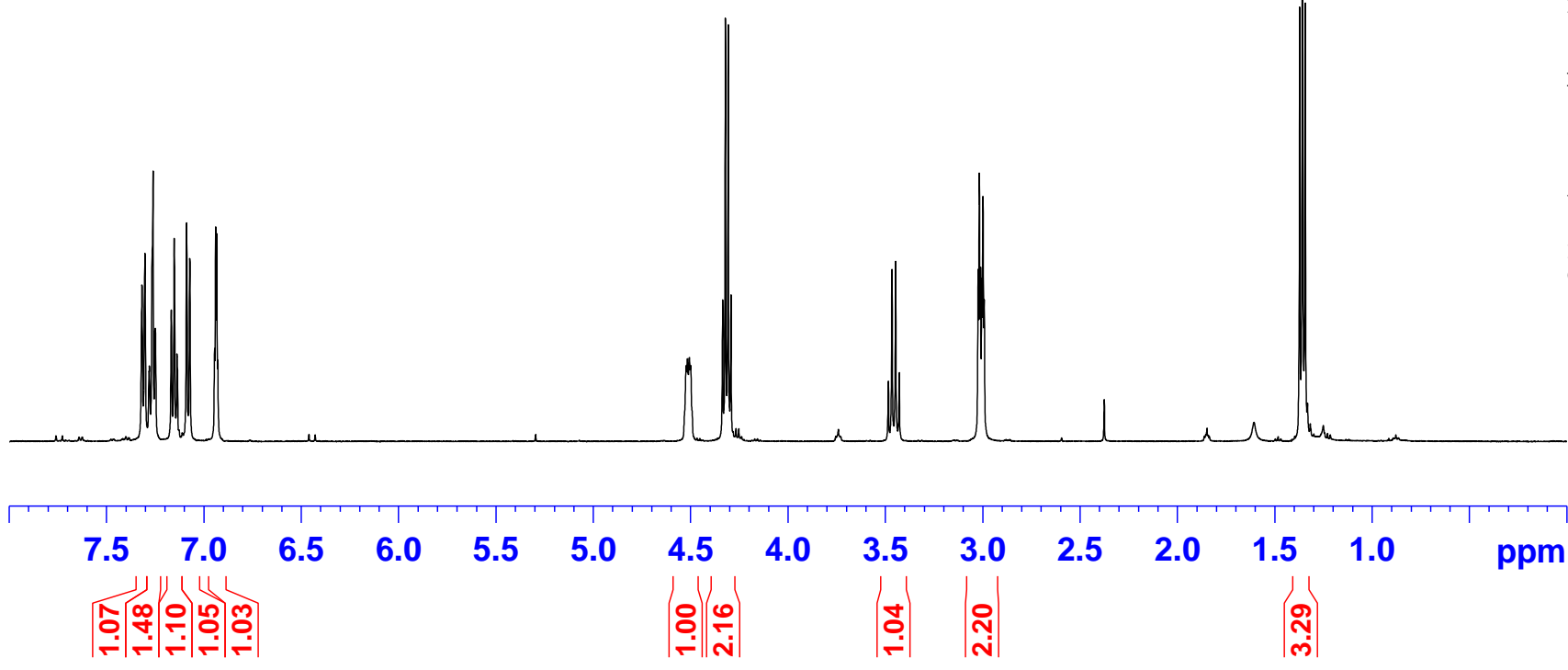

Current Data Parameters 


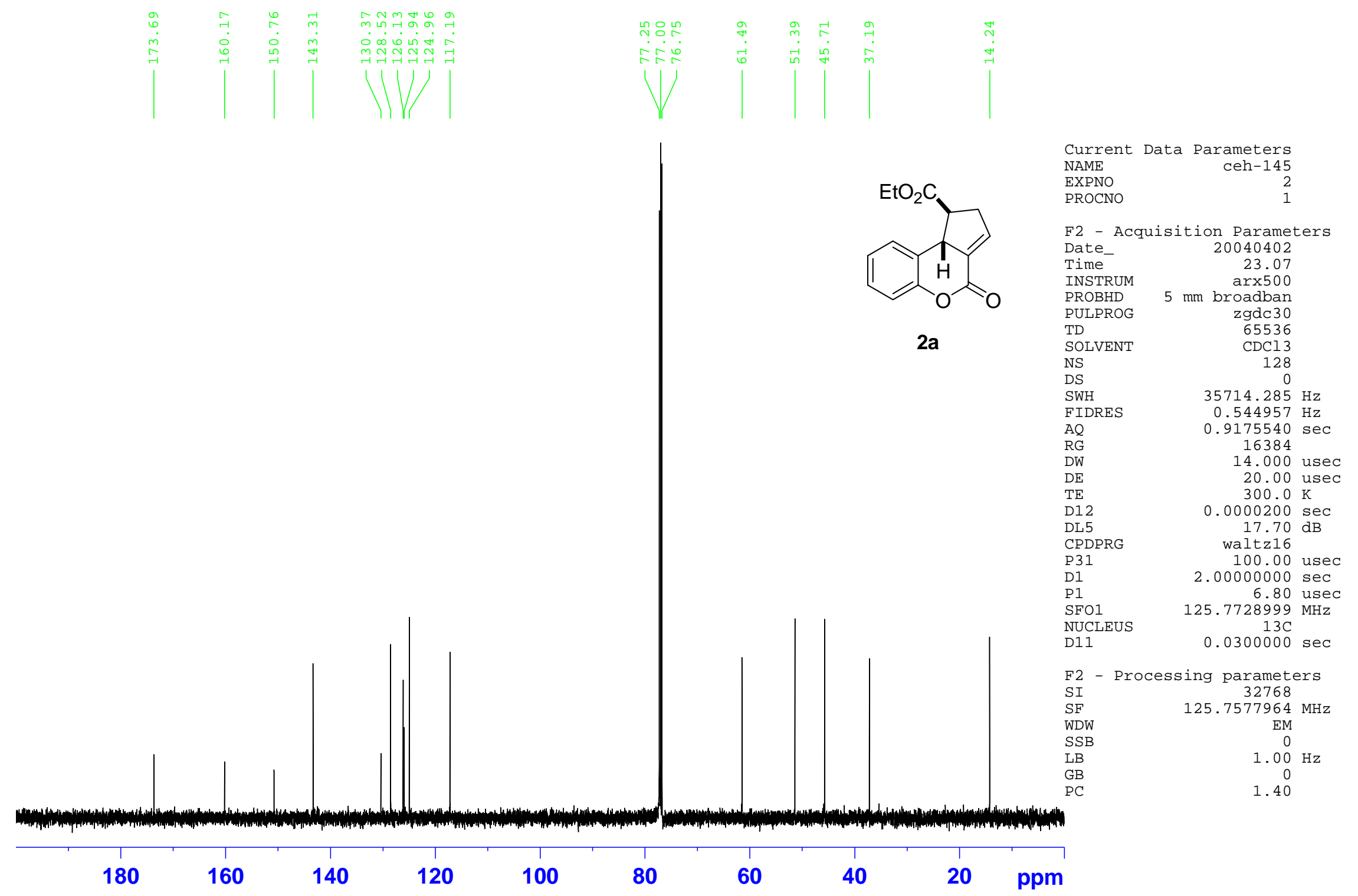




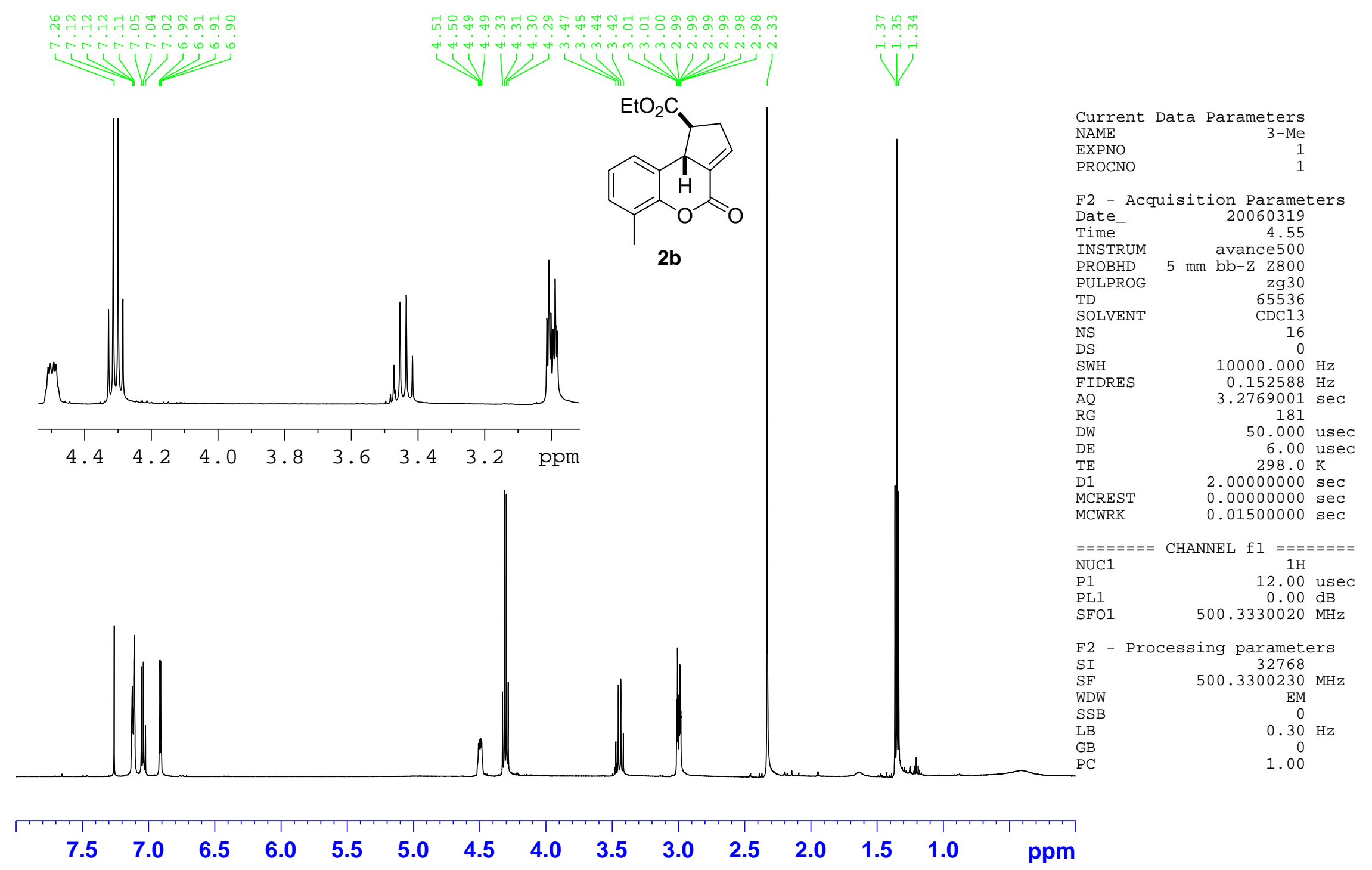




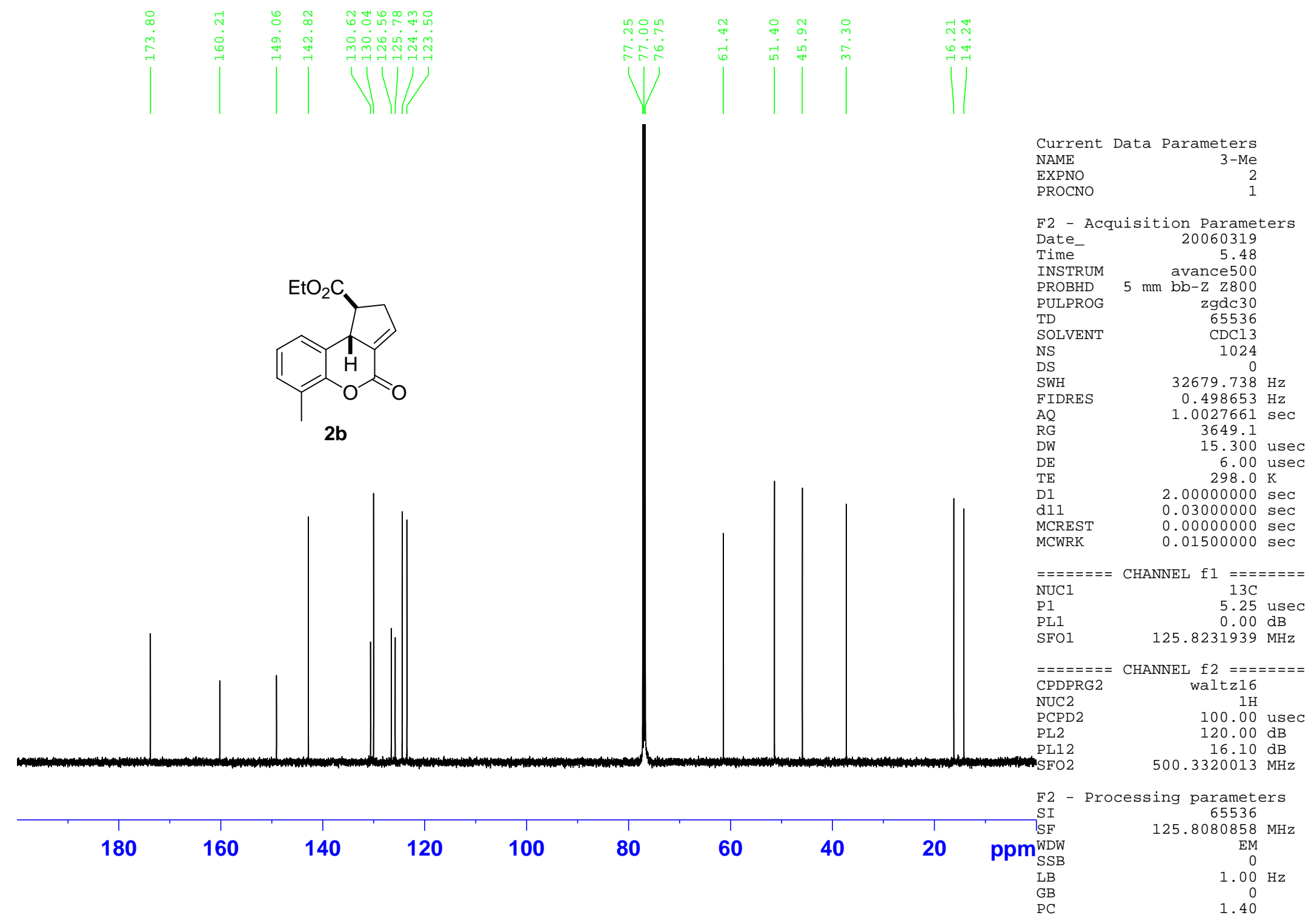




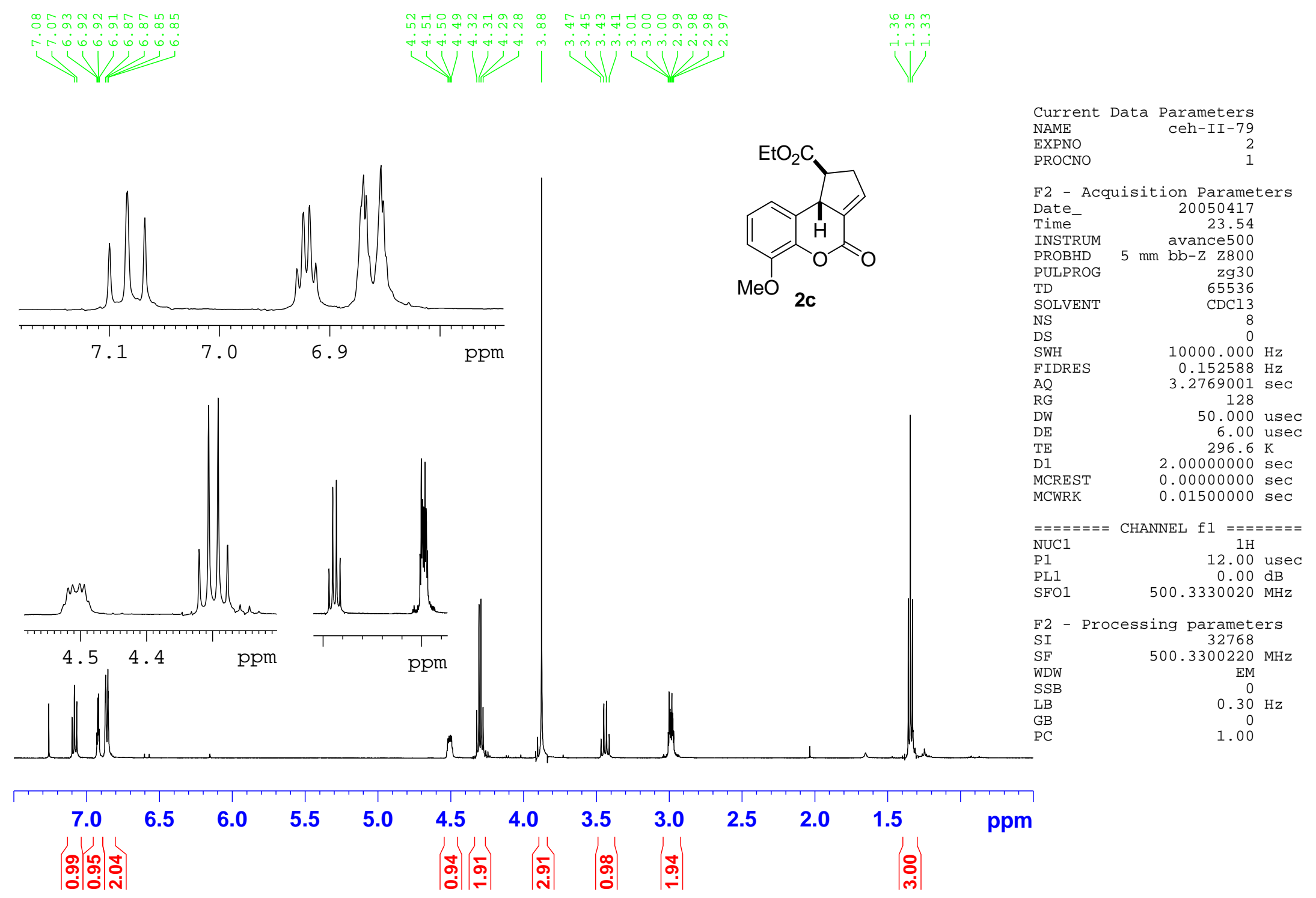




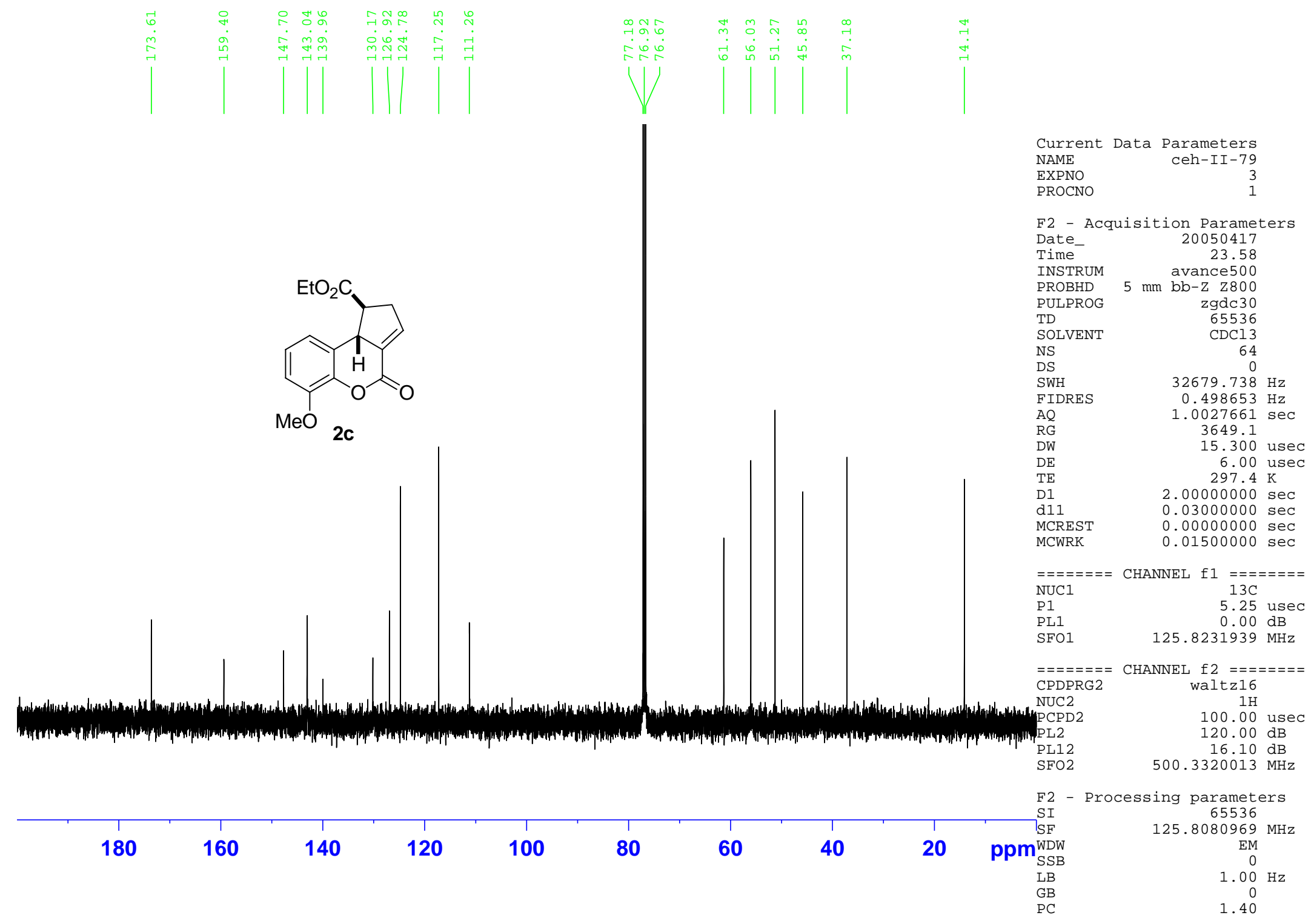




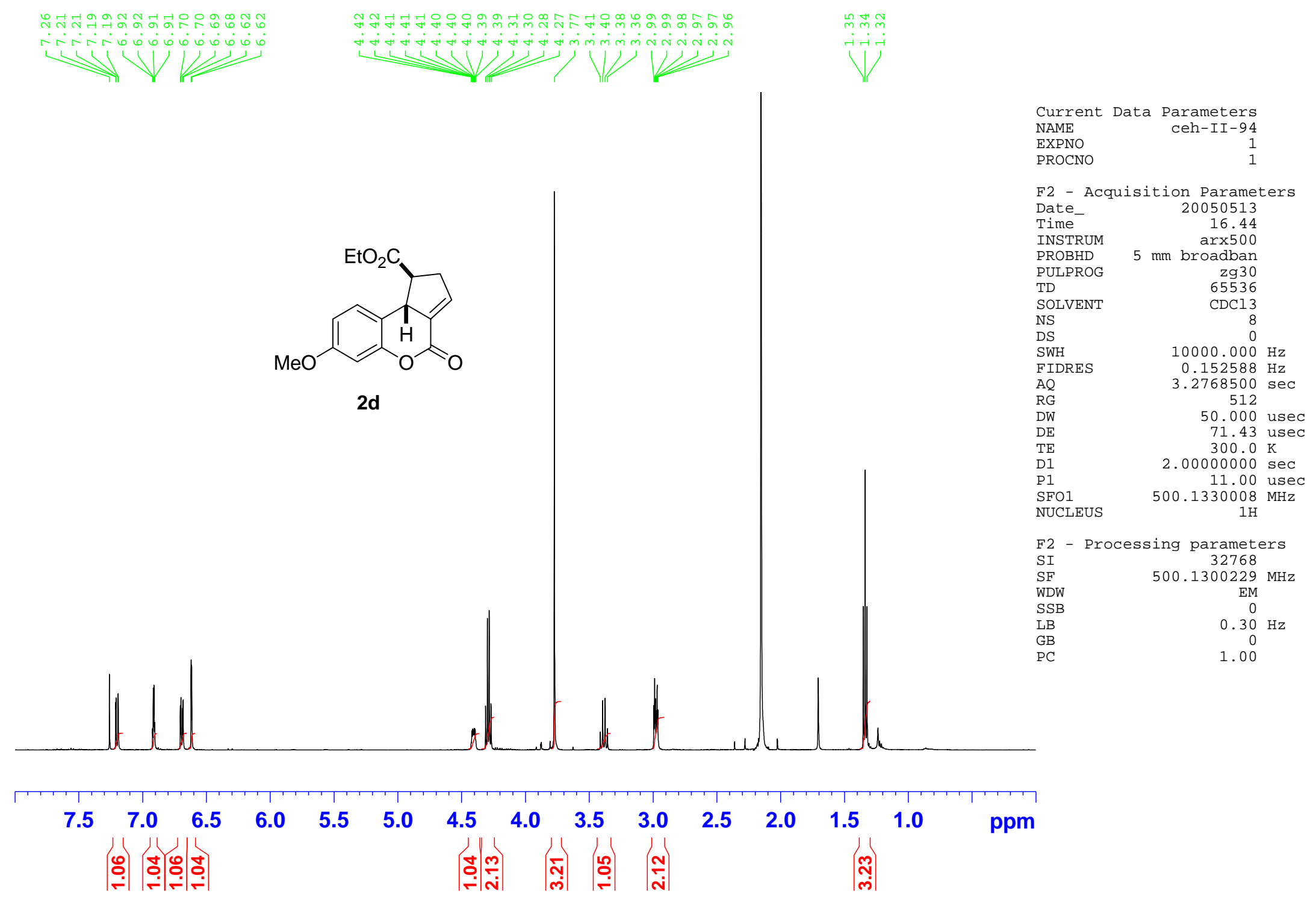




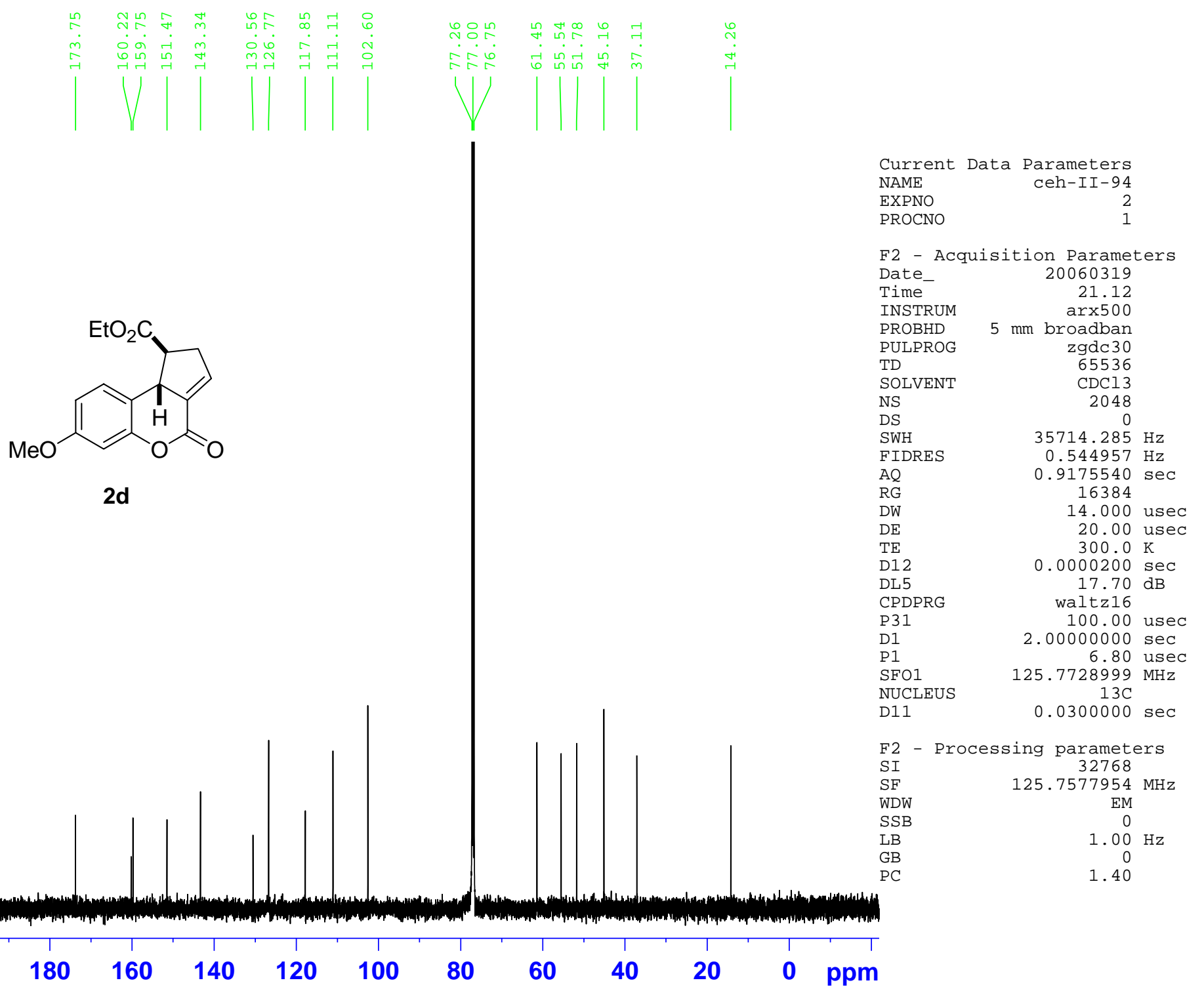




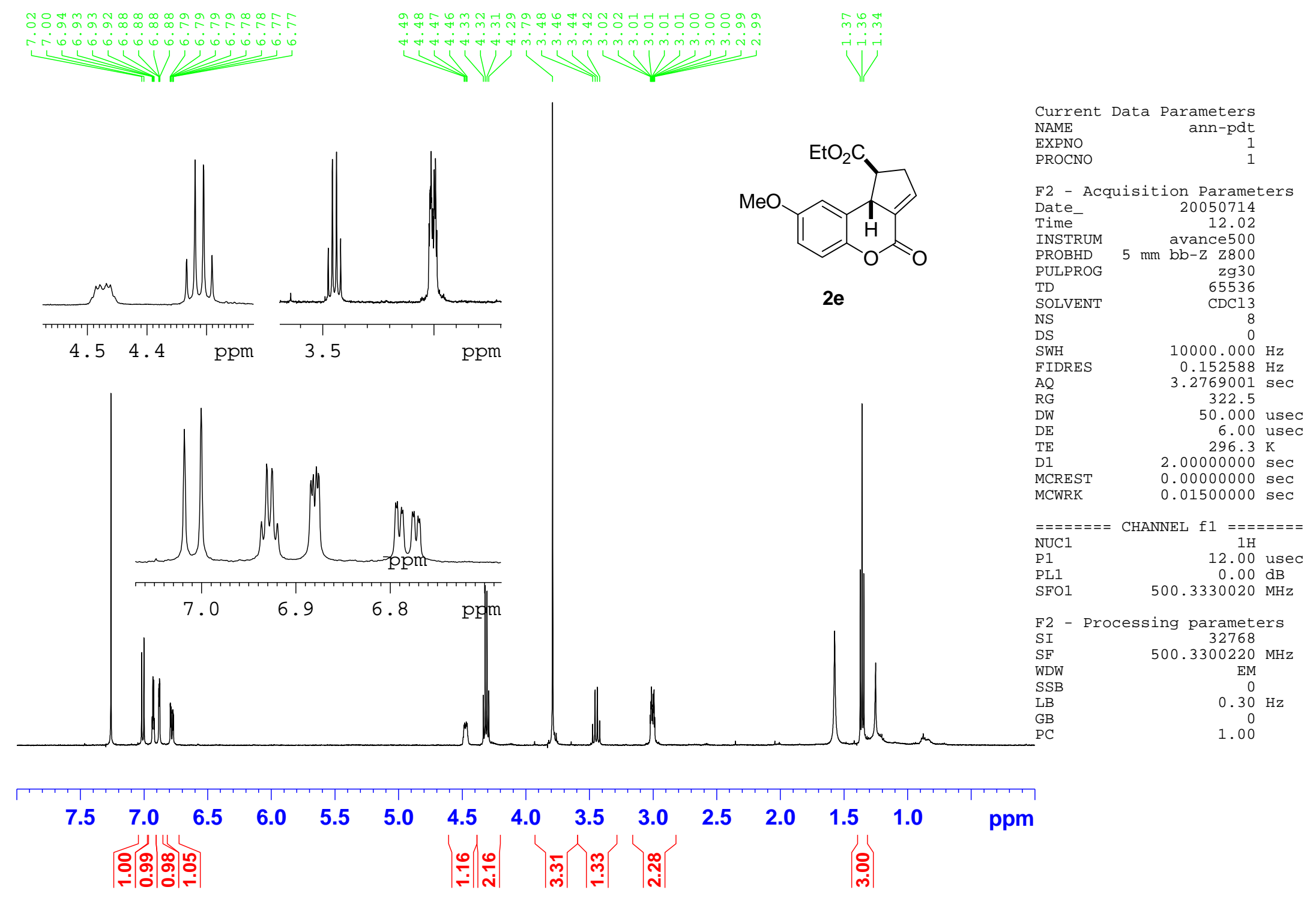




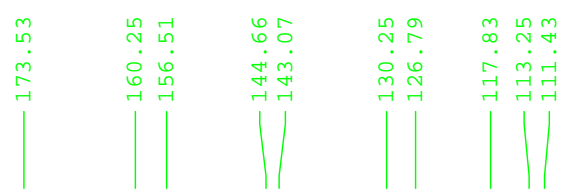

Current Data Parameters NAME ann-pdt

EXPNO

F2 - Acquisition Parameters

$\begin{array}{lr}\text { Date_- } & 20050714 \\ \text { Time } & 12.55\end{array}$

20050714
12.55

Time

PROBHD $5 \mathrm{~mm} \mathrm{bb-z} \mathrm{Z800}$

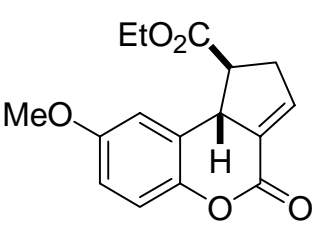

$2 e$
PULPROG

TD

NS

DS

SWH
FIDRES

FIDRES
$\mathrm{AQ}$

$\mathrm{AQ}$
$\mathrm{RG}$

RW

DE

TE

D1 1

d11

MCWRK

$========$ CHANNEL

NUC1

P1

PL1
SF01

$=======$ CHANNEL f2 $=======$ CPDPRG

PCPD2

$\mathrm{PL2} 2$

SF02

- Processing parameters

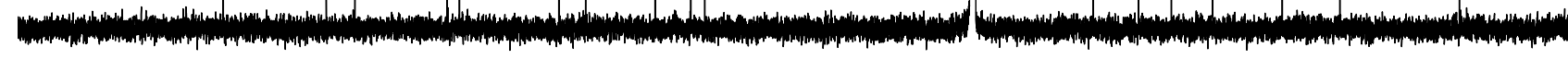

SF

SSB
LB

GC $\mathrm{zgdc} 30$
65536

$\mathrm{CDCl} 3$

1067

$32679.738 \mathrm{~Hz}$

1. $0027661 \mathrm{sec}$

15.300 usec 297.4 K

$000000000 \mathrm{sec}$

$03000000 \mathrm{sec}$

0.00000000 sec $=$ CHANNEL $\mathrm{f}$

$1=======$
$0.00 \mathrm{~dB}$ waltz16

100.00 usec

$120.00 \mathrm{~dB}$ $500.3320013 \mathrm{MHz}$
125. $8080969 \mathrm{MHz}$

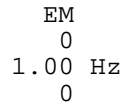

1. 


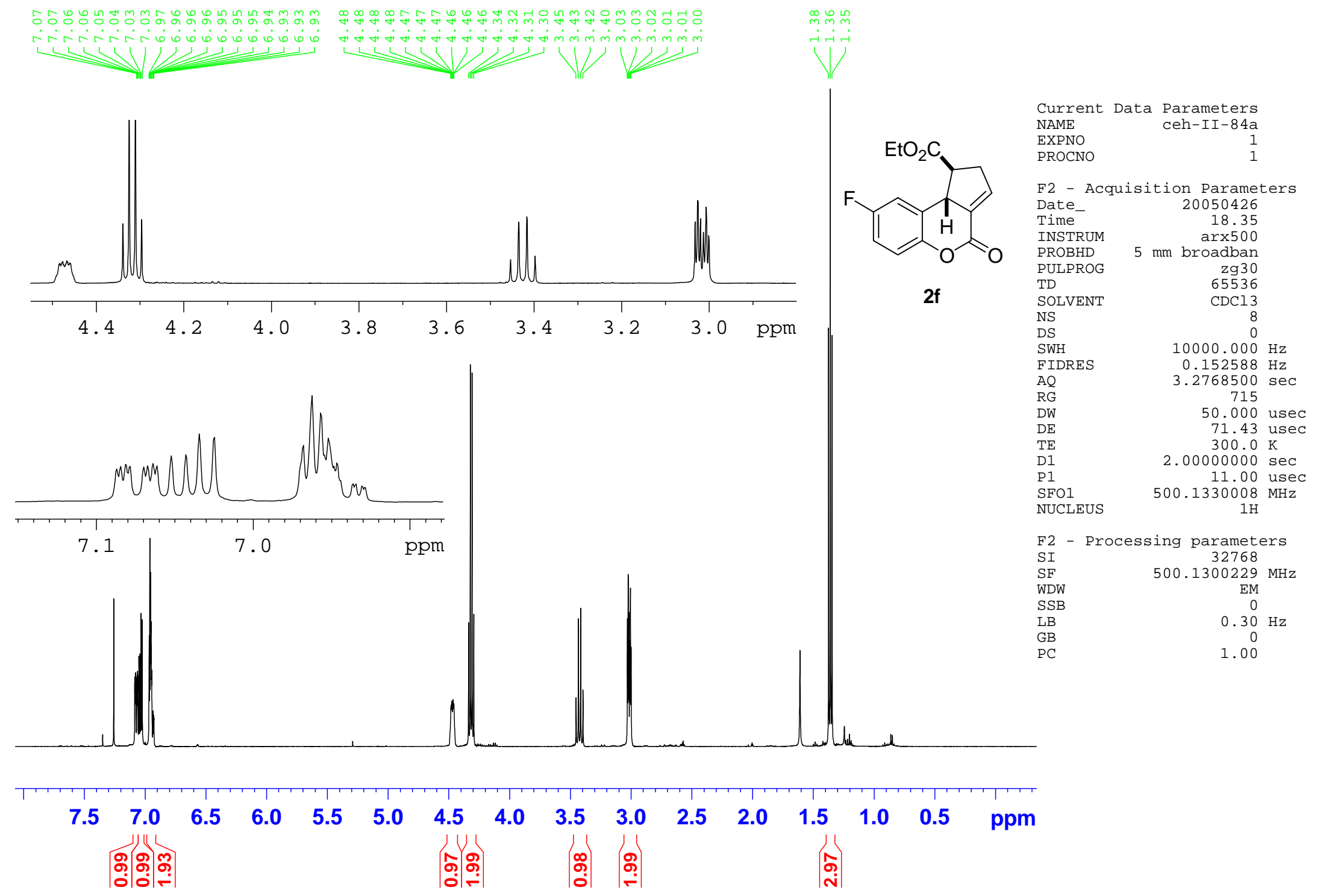




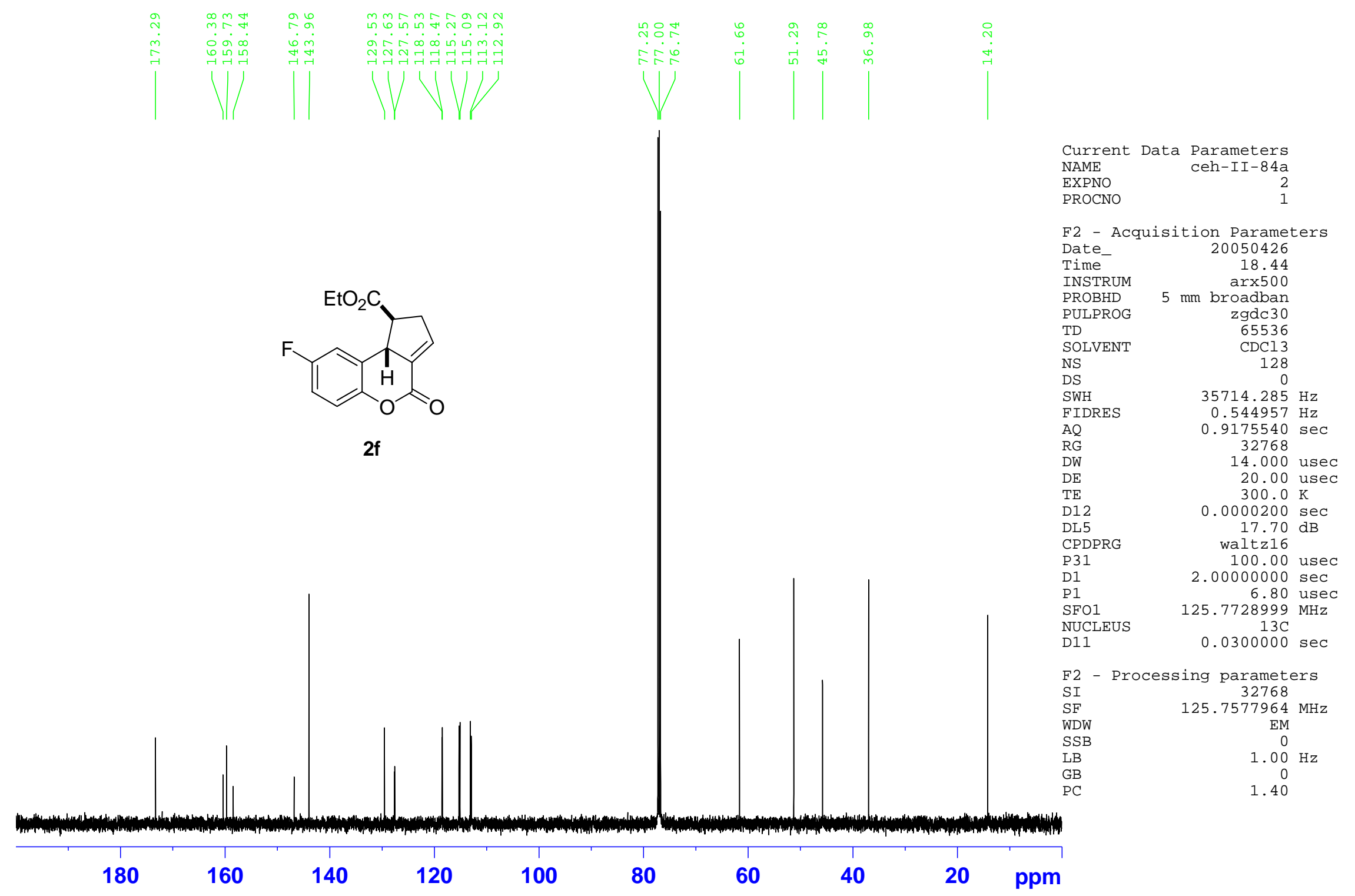




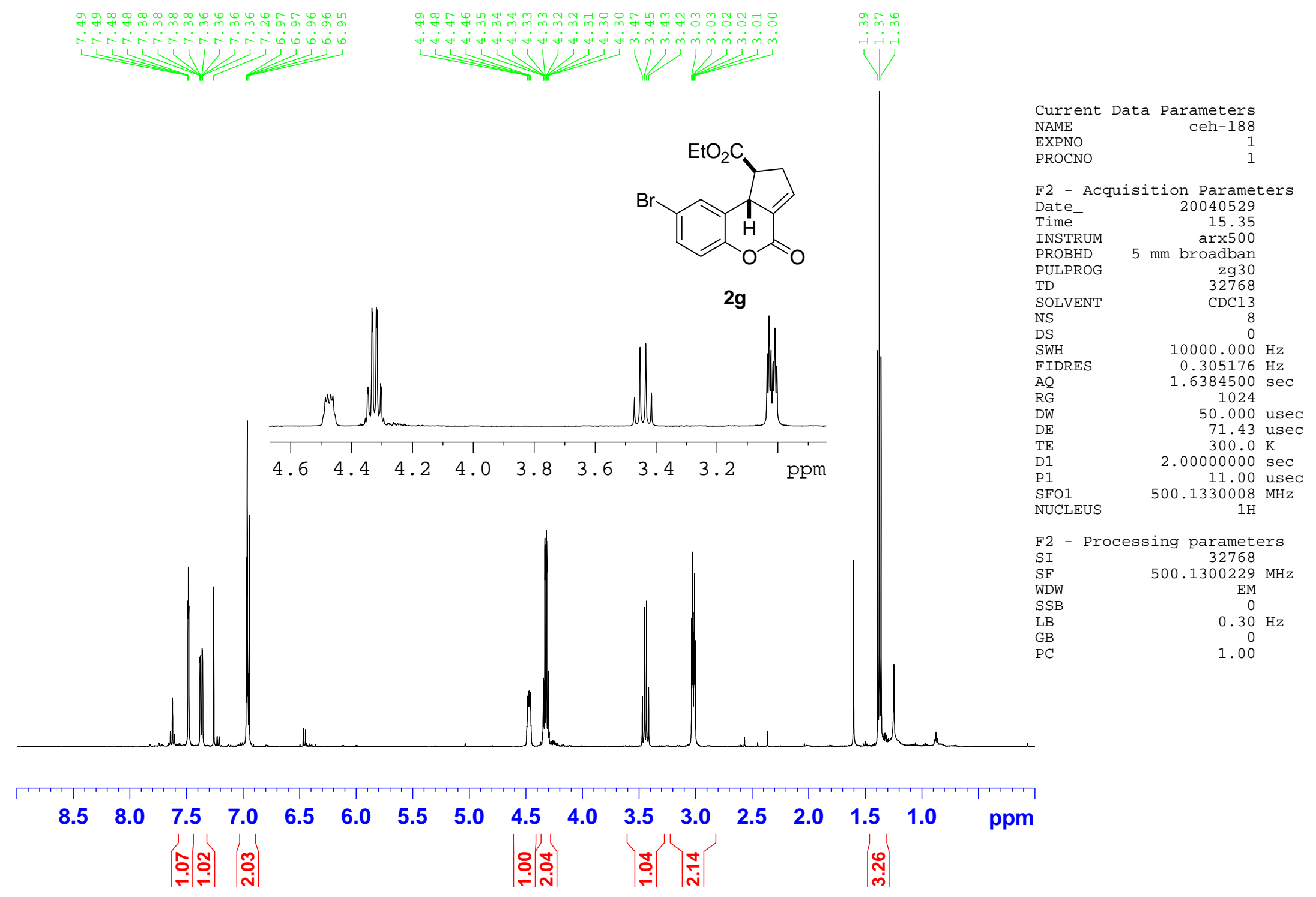




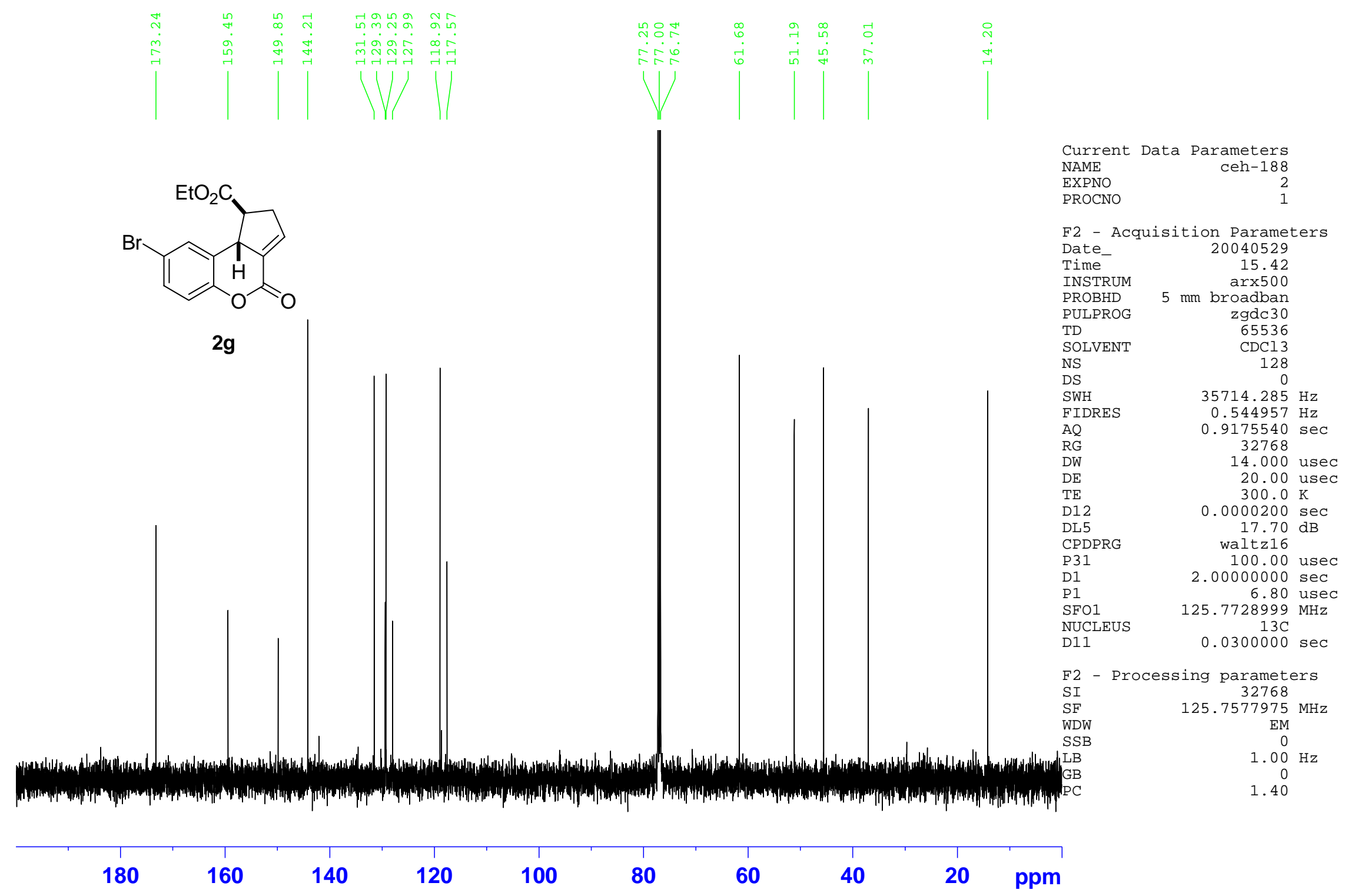




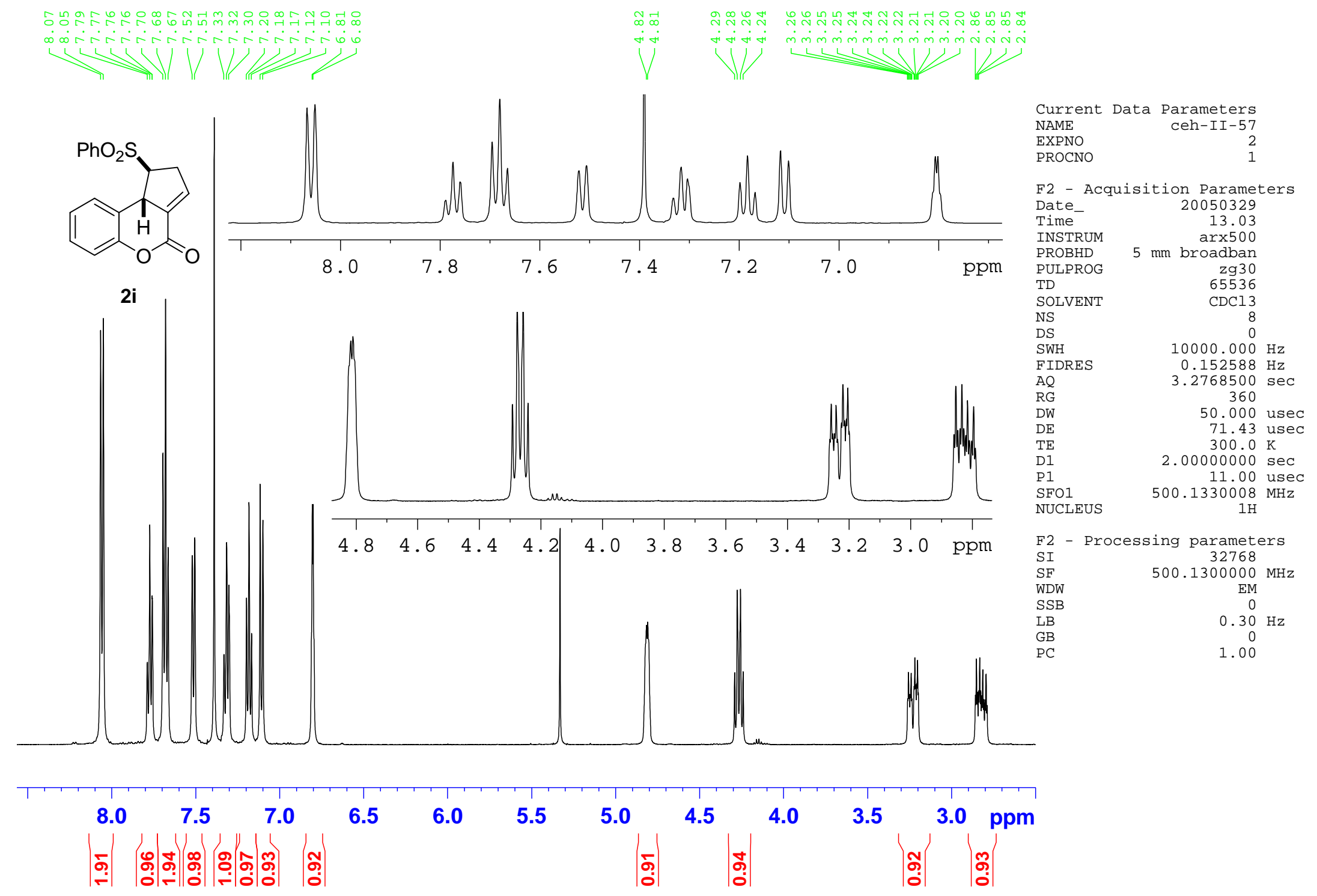




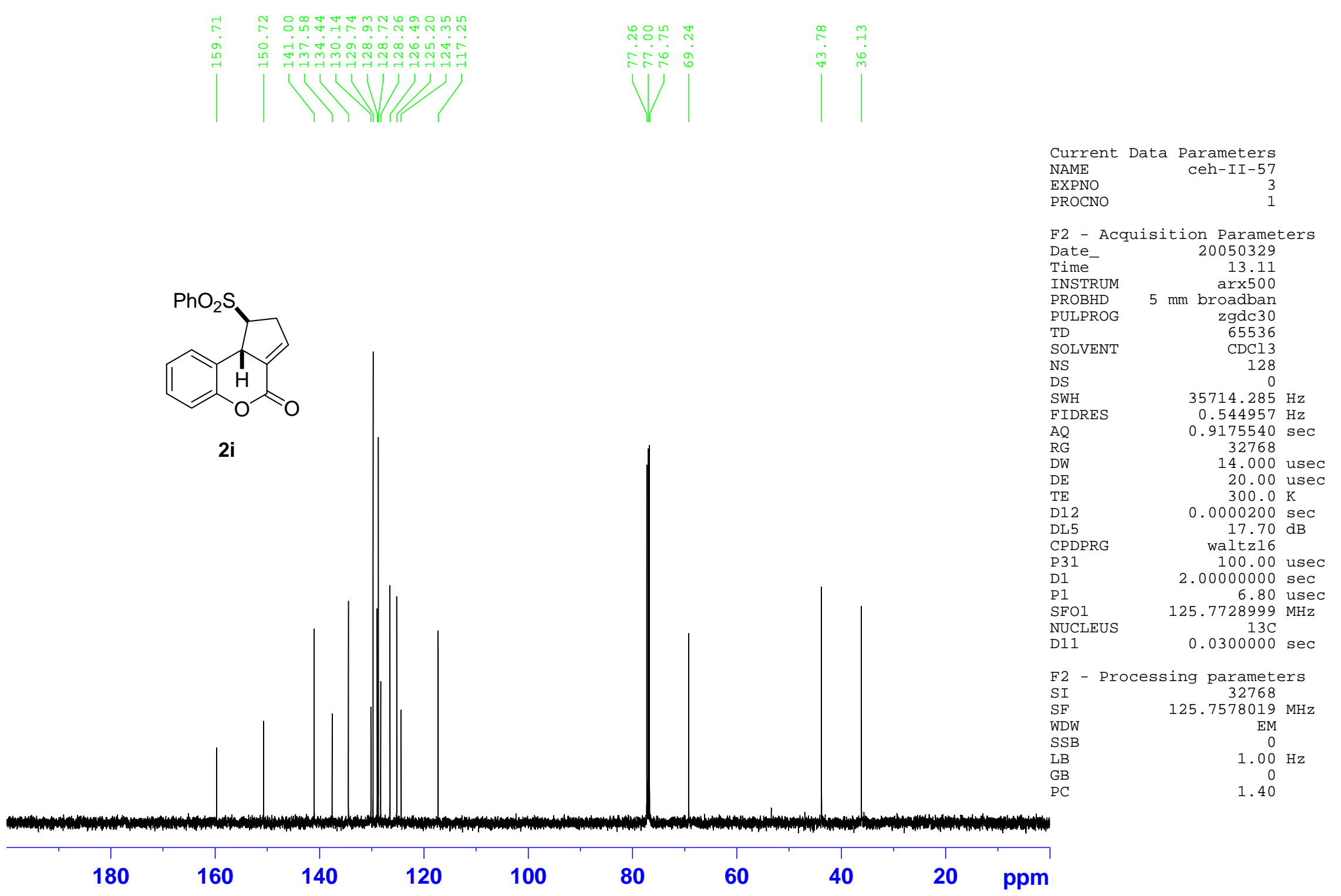




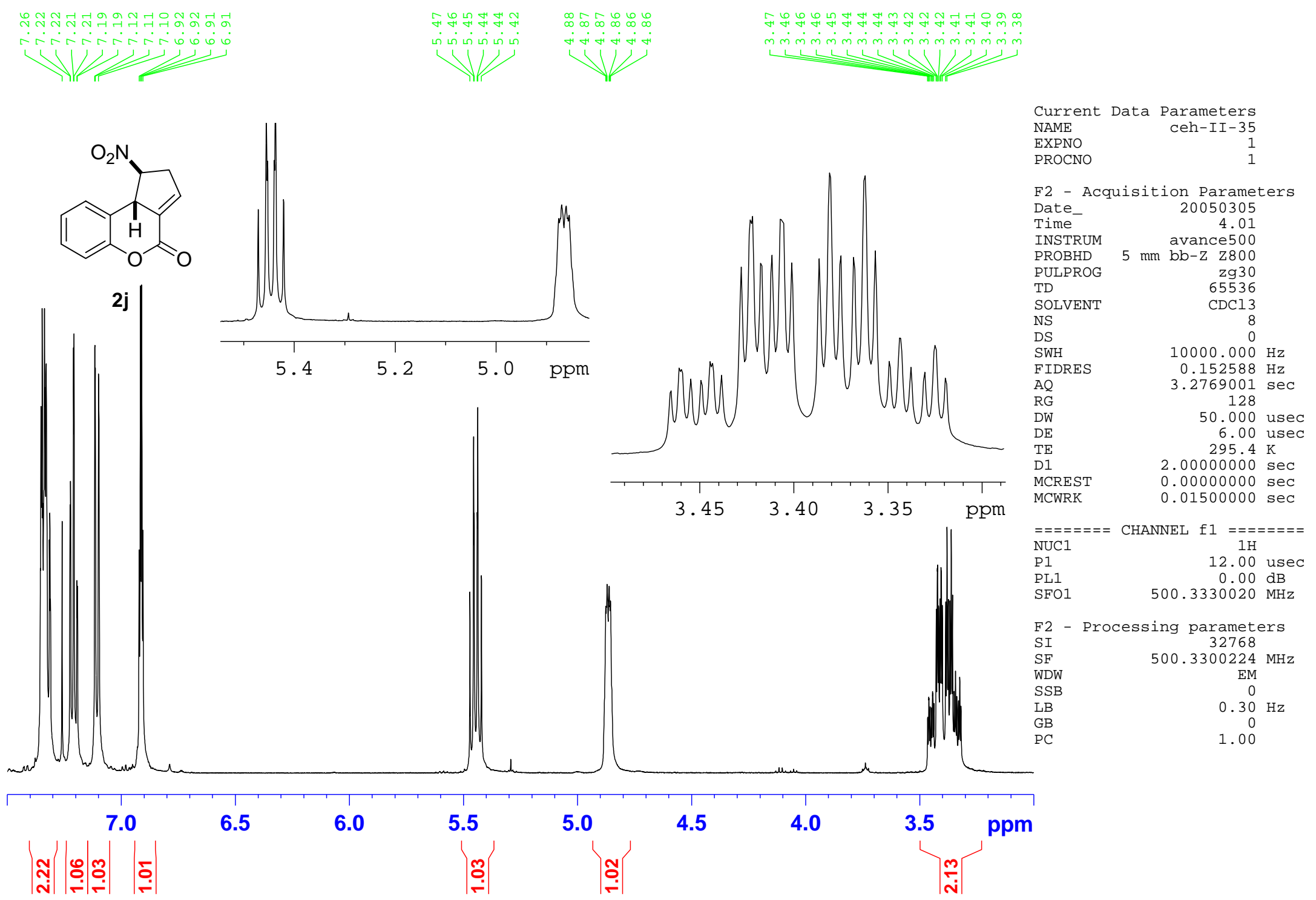



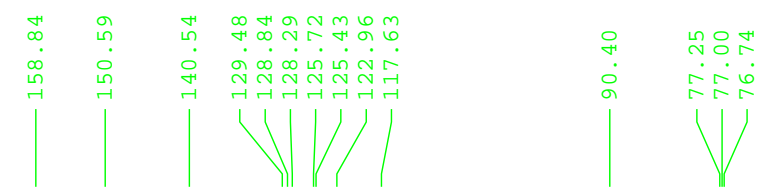
Current Data Parameters
NAME ceh-II-35

EXPNO

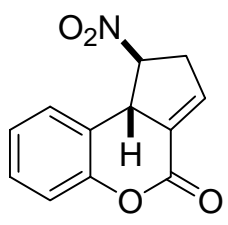

F2 - Acquisition Parameters
Date 20050305

Date

Time

PROBHD $5 \mathrm{~mm}$ bb-Z 2800 $5 \mathrm{~mm}$ bb-Z Z800 PULPROG Zgdc30

SOLVENT

NS

DS

SWH
FIDRES

2j

FIDRES $\quad 32679.738 \mathrm{~Hz}$

AQ $1.0027661 \mathrm{sec}$

RG

DW 15.300 usec

DE

TE

D1 296.4 K

d11 $0.03000000 \mathrm{sec}$

MCREST $\quad 0.00000000 \mathrm{sec}$ MCWRK $\quad 0.01500000 \mathrm{sec}$

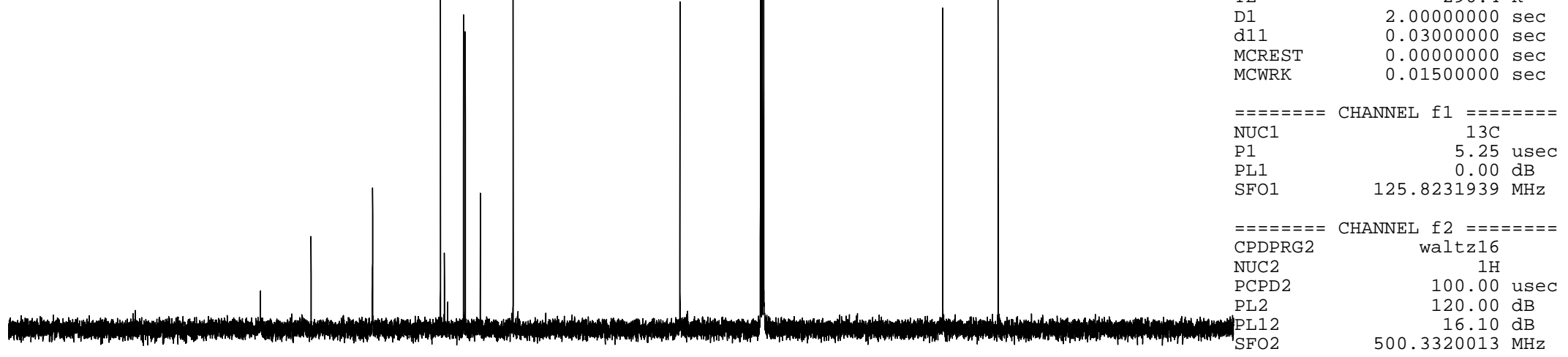

F2 - Processing parameters

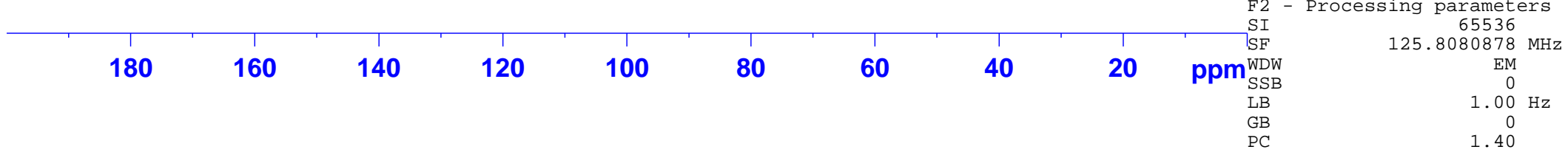




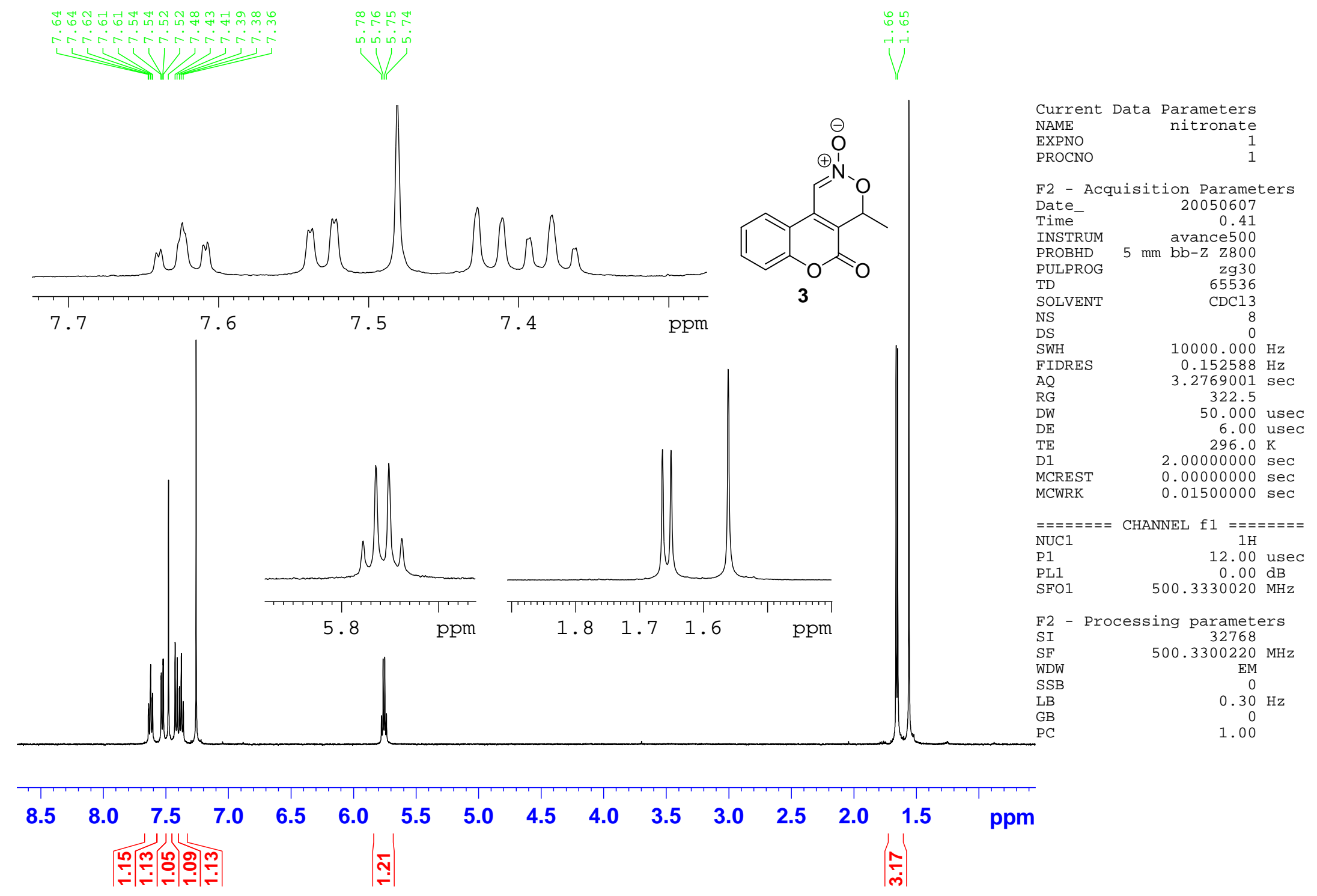




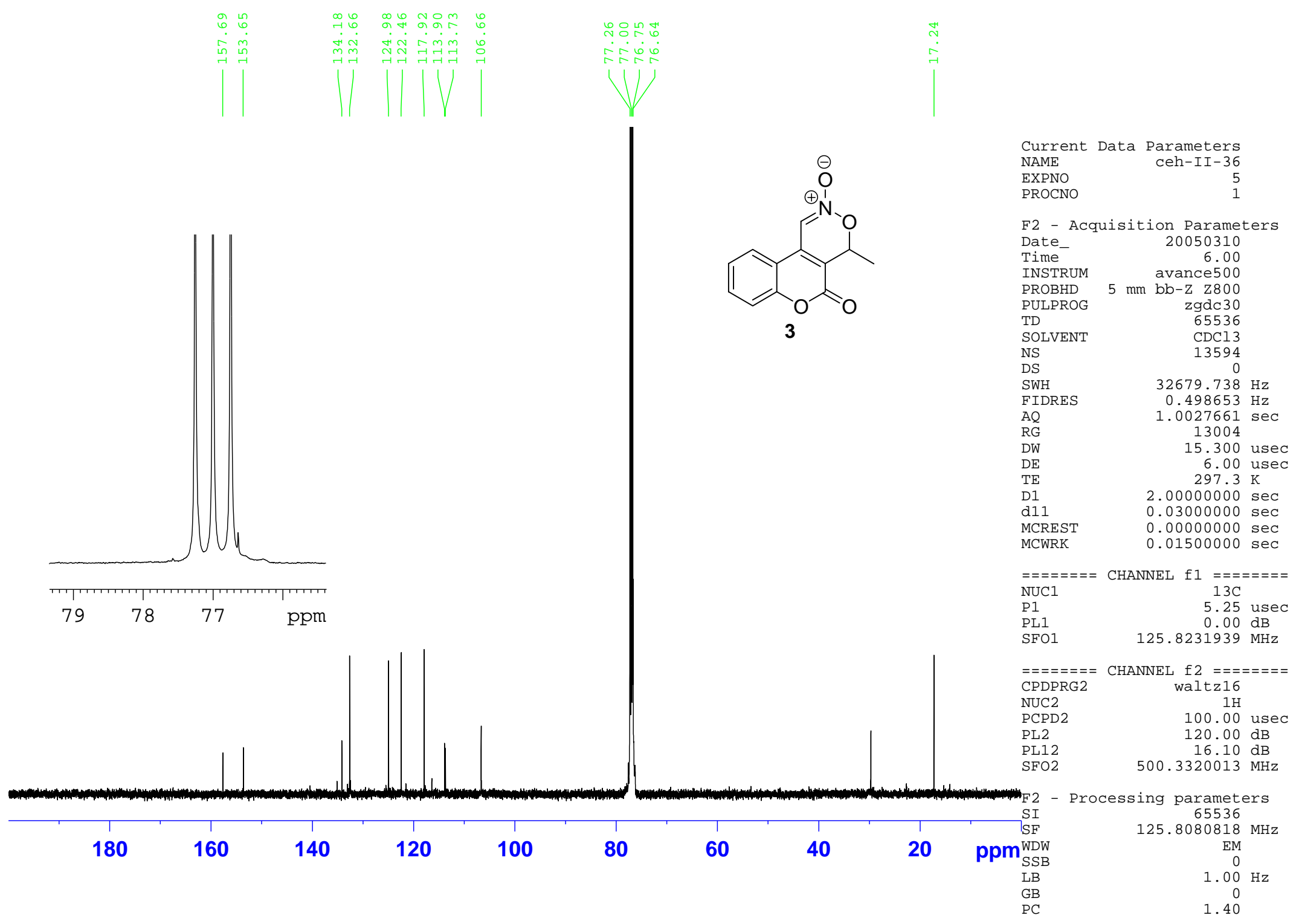




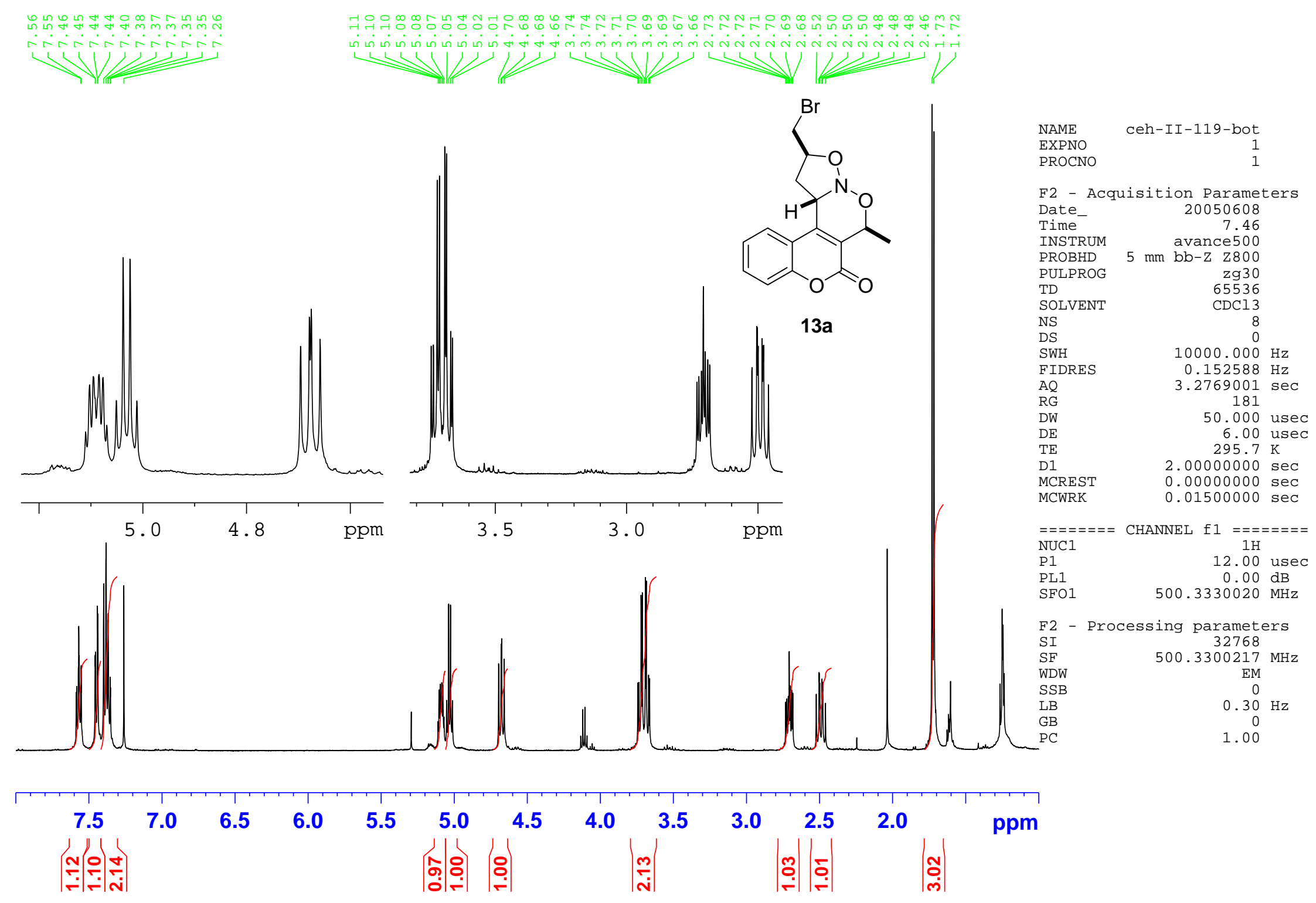




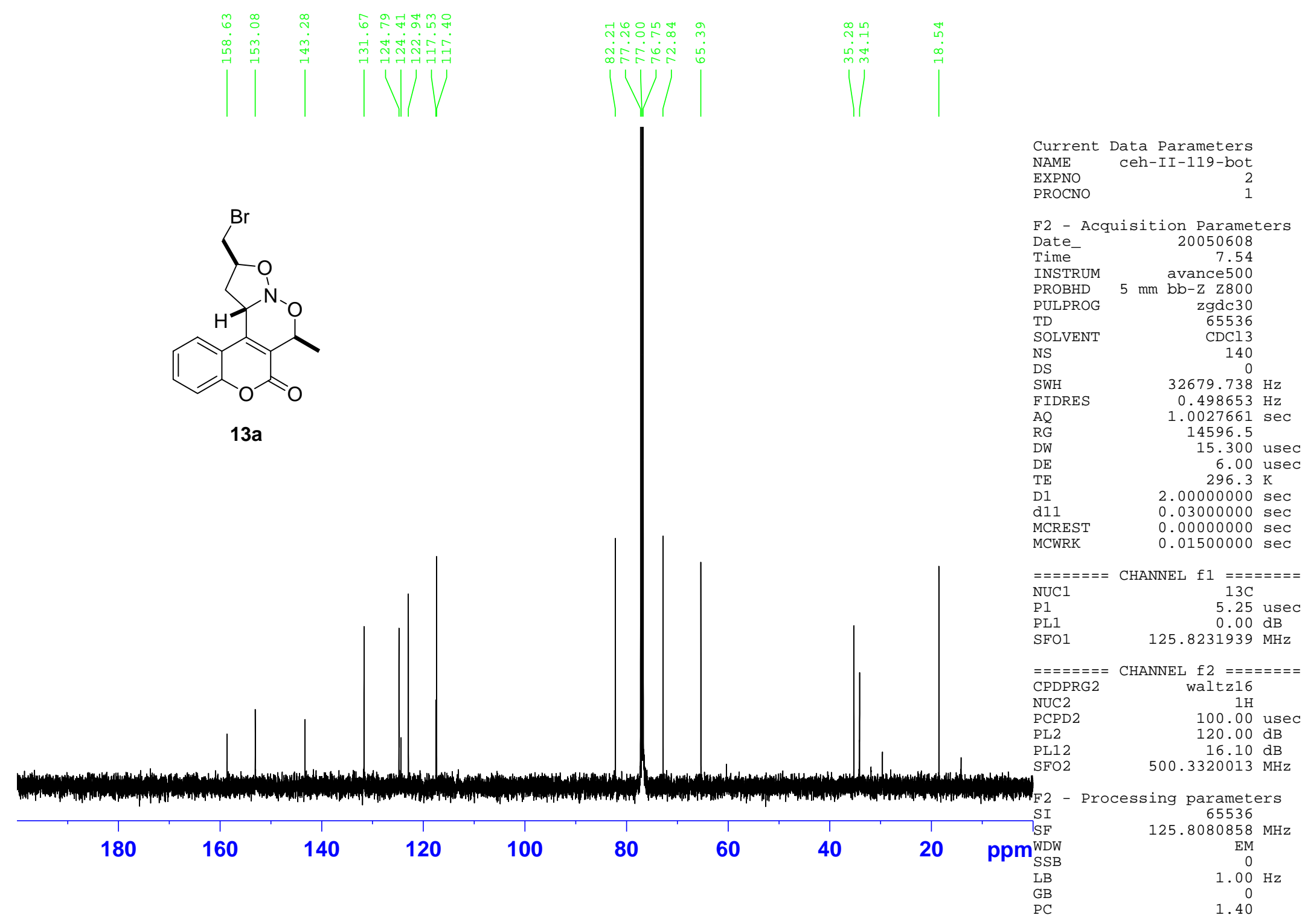



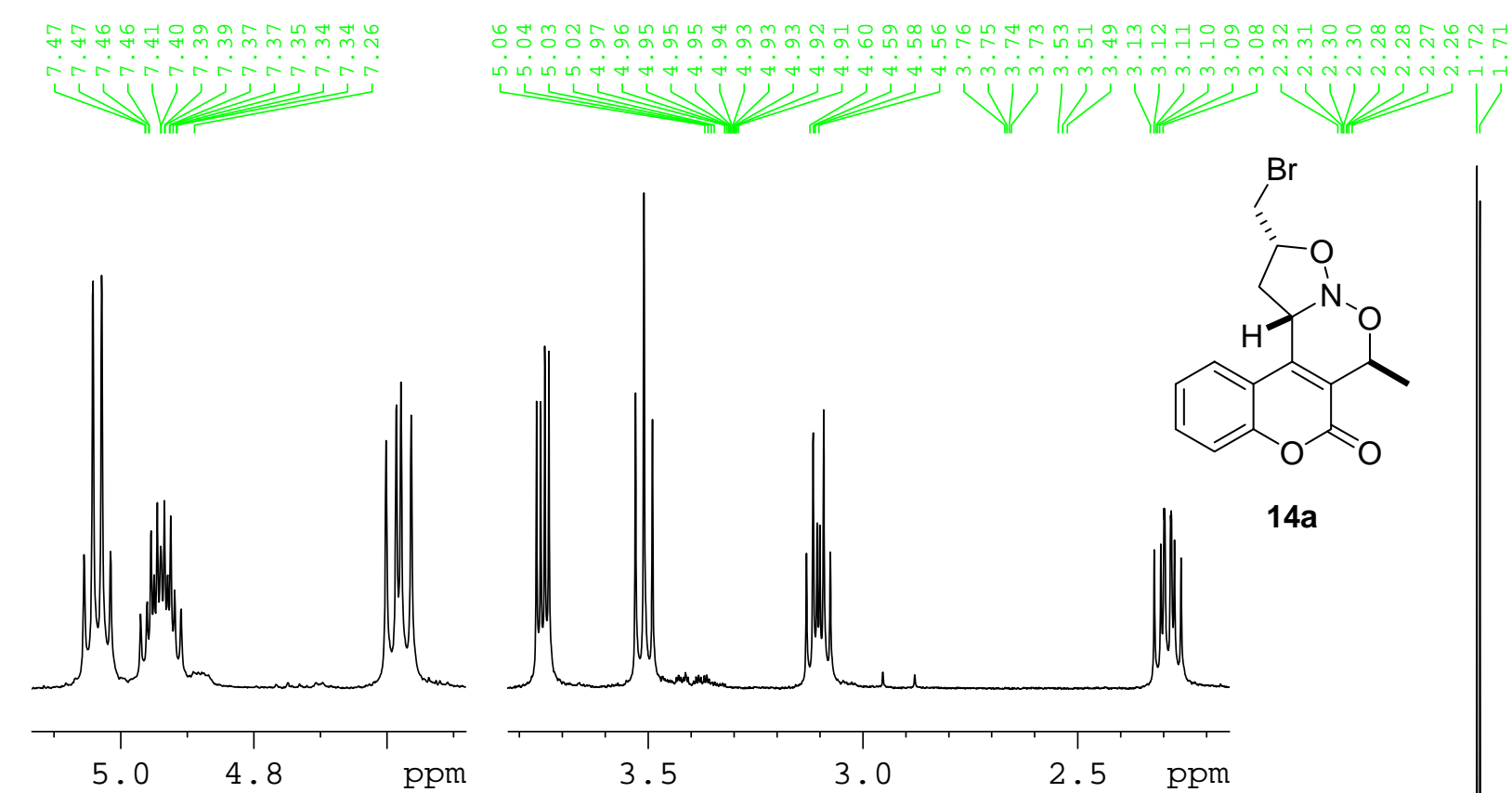

Current Data Parameters

NAME ceh-II-119-top

EXPNO

PROCNO

\section{1}

F2 - Acquisition Parameters

Date_ 20050608

$\begin{array}{lr}\text { Time } & 7.28 \\ \text { INSTRUM } & \text { avance500 }\end{array}$

$5 \mathrm{~mm}$ bb-Z Z800

PULPROG $\quad \mathrm{zg} 30$

65536

SOLVENT

NS

$\begin{array}{lr}\text { DS } & 0 \\ \text { SWH } & 10000.000 \mathrm{~Hz}\end{array}$

FIDRES $\quad 0.152588 \mathrm{~Hz}$

AQ $\quad 0.152588 \mathrm{~Hz}$

$A Q$

$\mathrm{RG}$

DW

DE

203.2

50.000 usec 60.00 usec
6.00

TE $\quad 295.5 \mathrm{~K}$

D1 $\quad 2.0000000 \mathrm{Sec}$ $\begin{array}{ll}\text { MCREST } & 0.00000000 \mathrm{sec} \\ \text { MCWRK } & 0.01500000 \mathrm{sec}\end{array}$

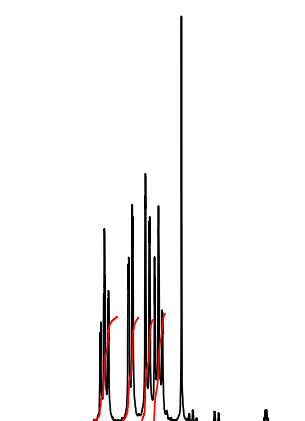

3.5

$2.5 \mathrm{ppm}$

$\begin{array}{lr}=======\text { CHANNEL } \mathrm{f} 1 \text { ======= } \\ \text { NUC1 } & 1 \mathrm{H} \\ \text { P1 } & 12.00 \mathrm{usec} \\ \text { PL1 } & 0.00 \mathrm{~dB} \\ \text { SF01 } & 500.3330020 \mathrm{MHz}\end{array}$

$\begin{array}{lr}\text { PF01 } & 0.00 \mathrm{~dB} \\ & 500.3330020 \mathrm{MHz}\end{array}$

F2 - Processing parameters

SI $\quad 32768$

SF $\quad 500.3300220 \mathrm{MHZ}$

WDW EM

SSB

LB

GB

$\mathrm{EM}$
$0.30 \mathrm{~Hz}$
0

$1 . \odot \odot$

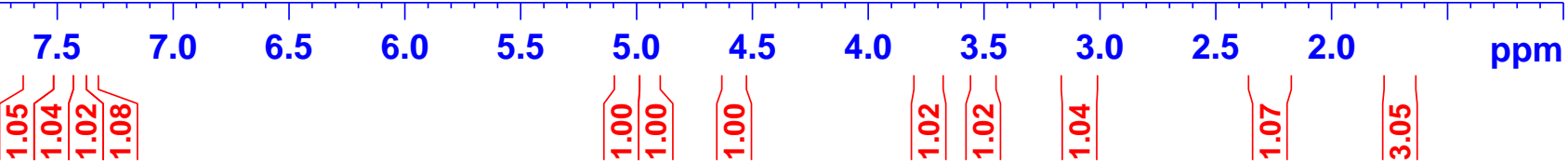

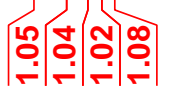

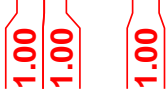

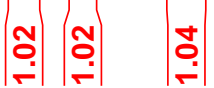

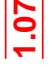

ले| 


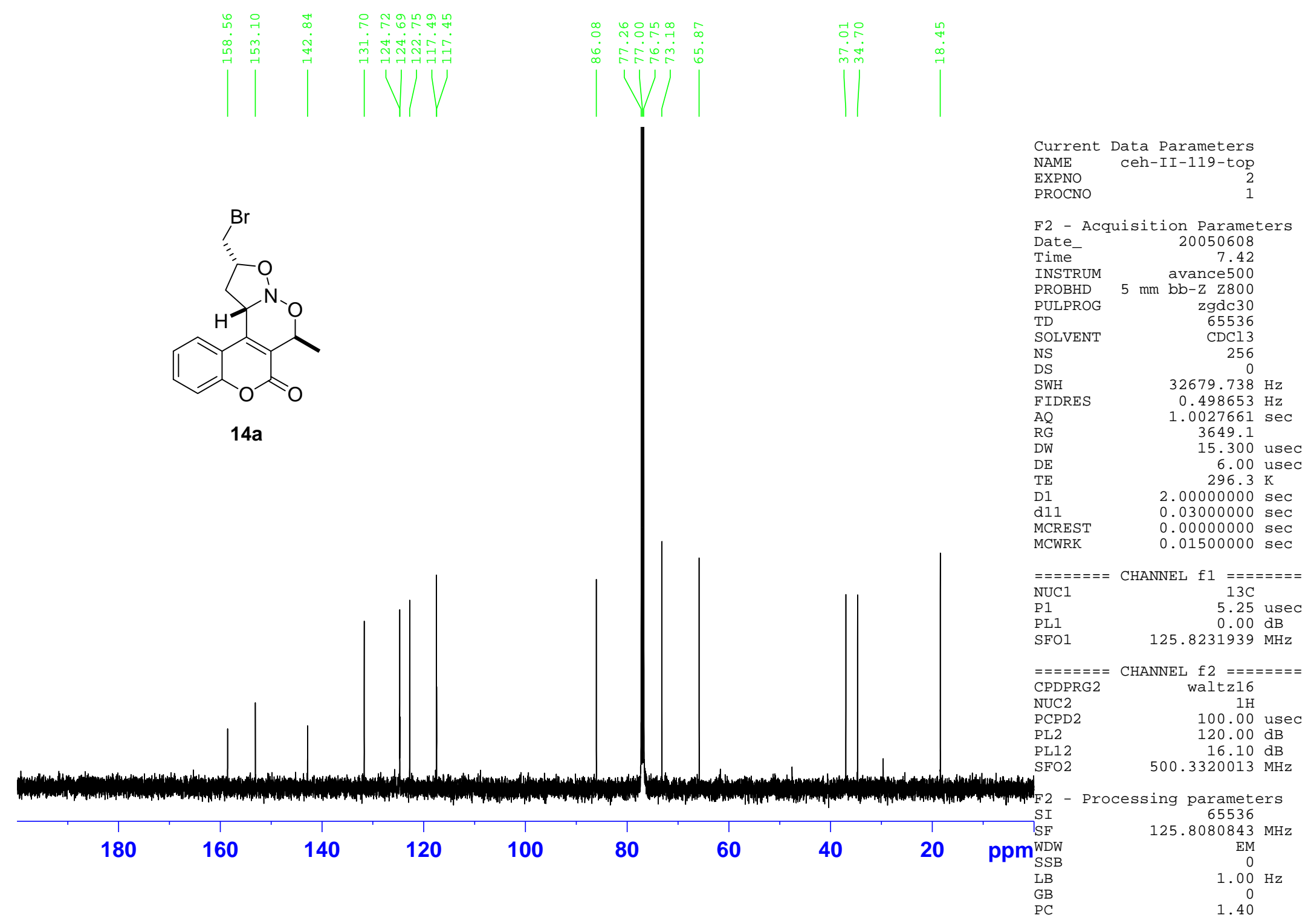




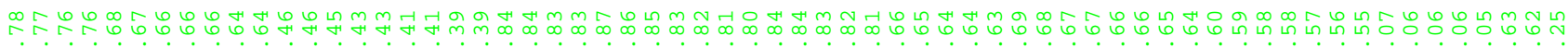

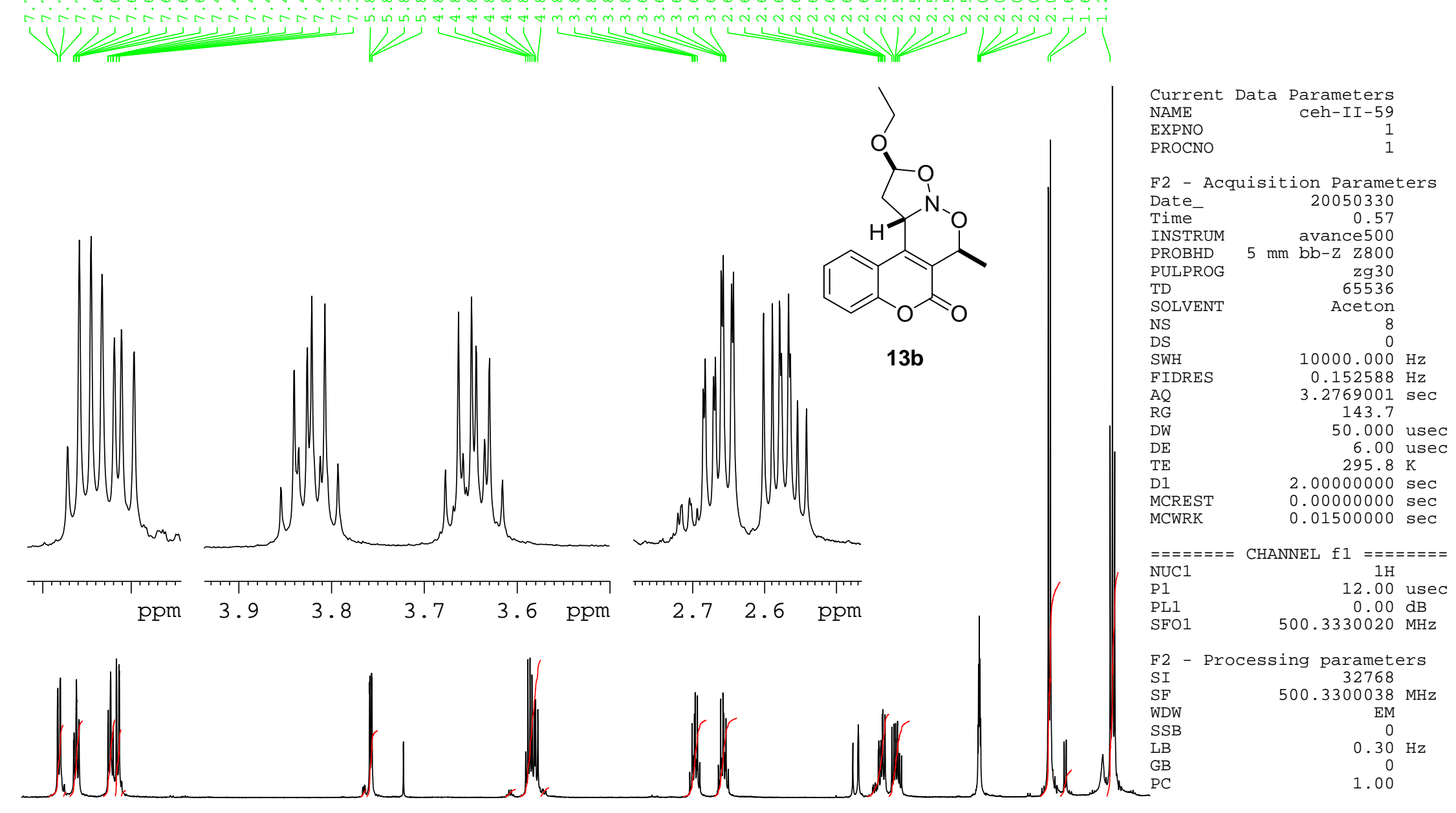

$7.5 \quad 7.0$

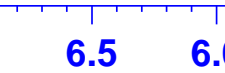

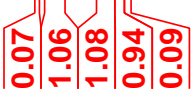

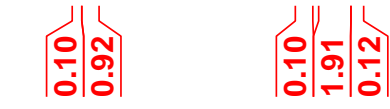

4.5

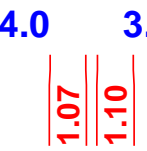

3.5

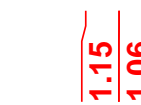

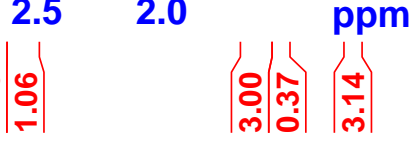




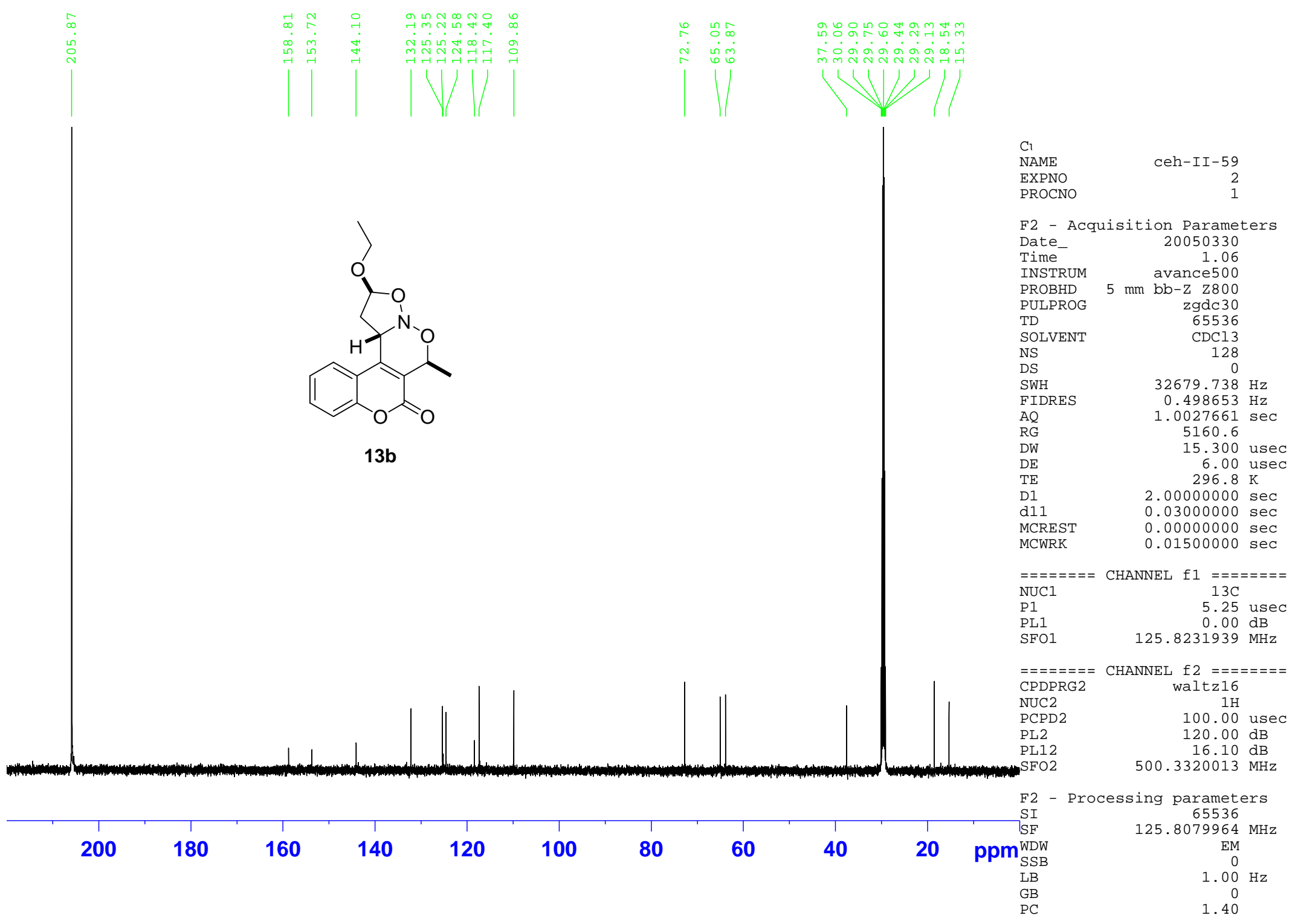



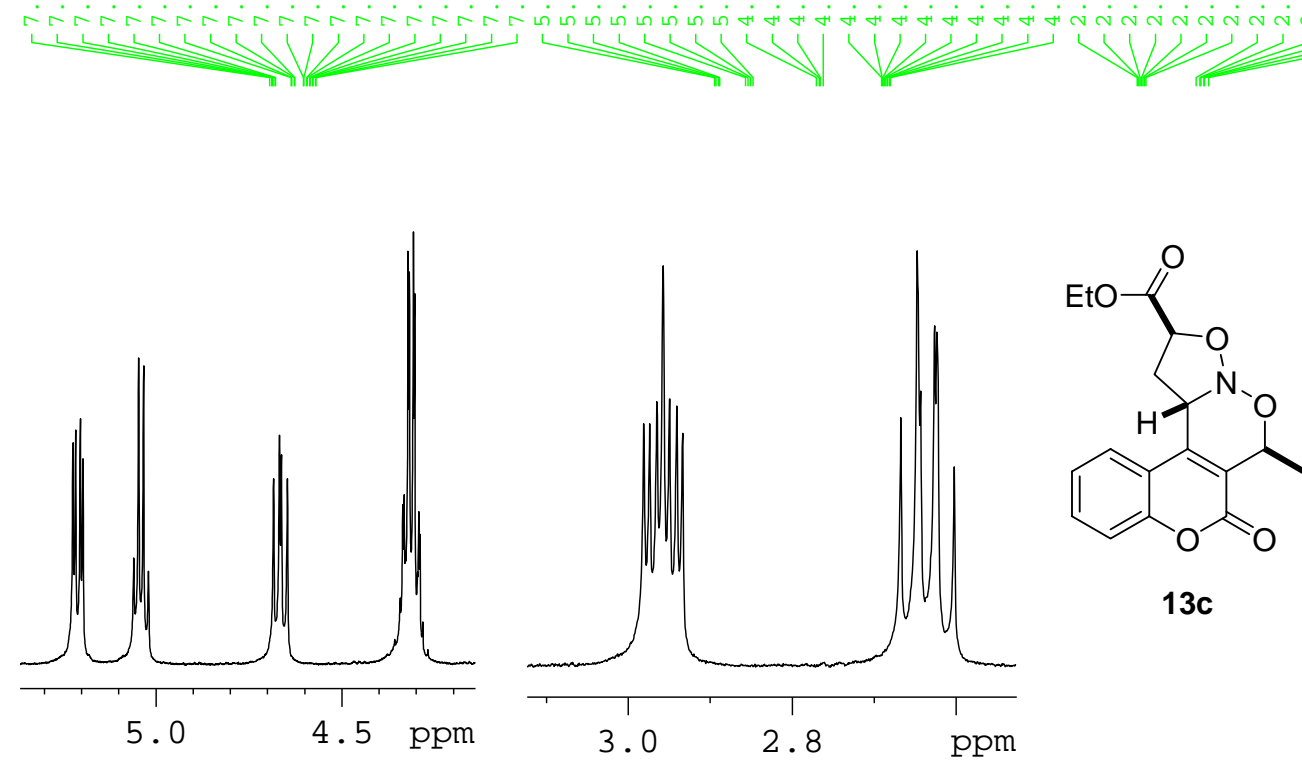

$13 c$
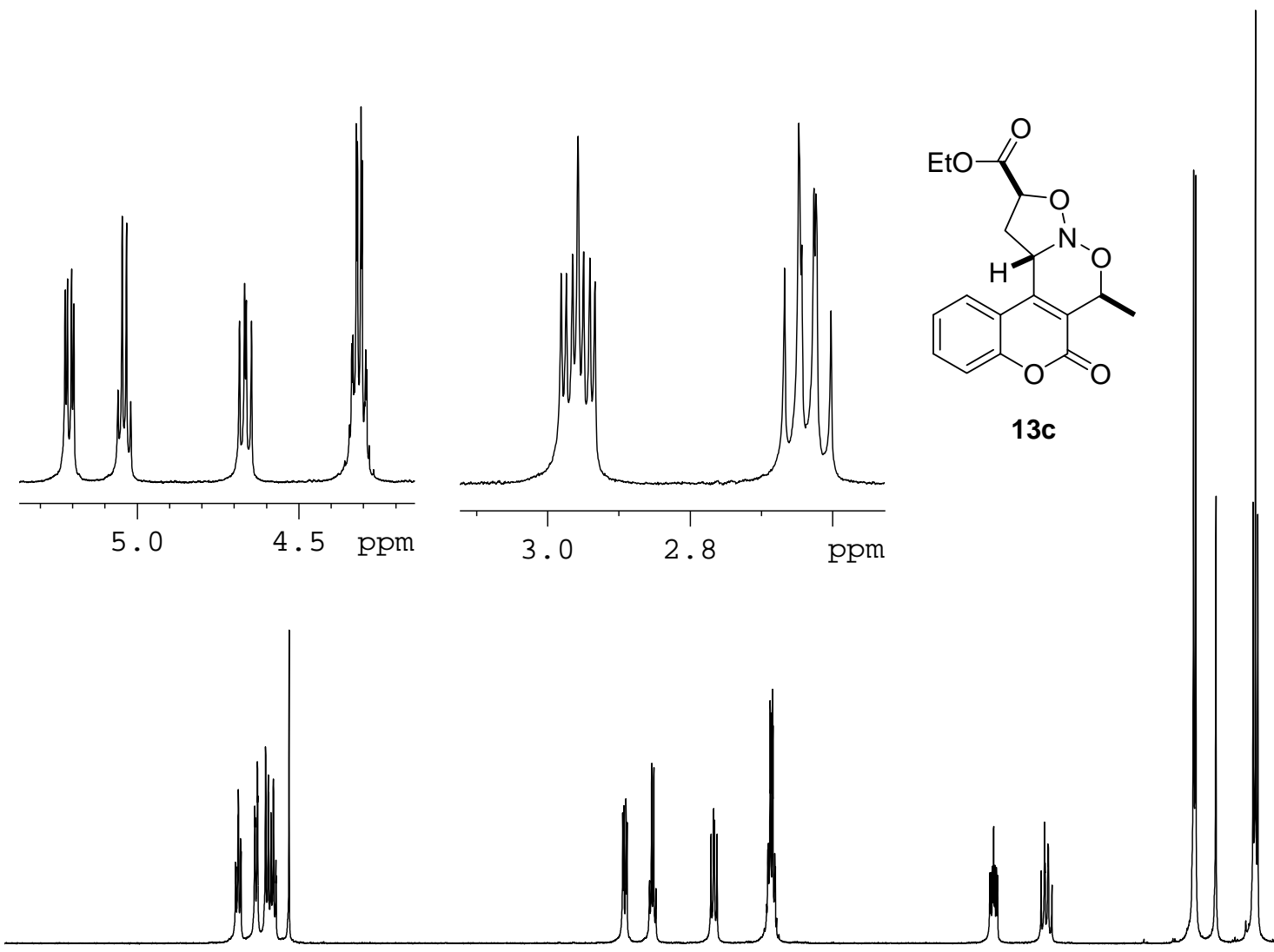


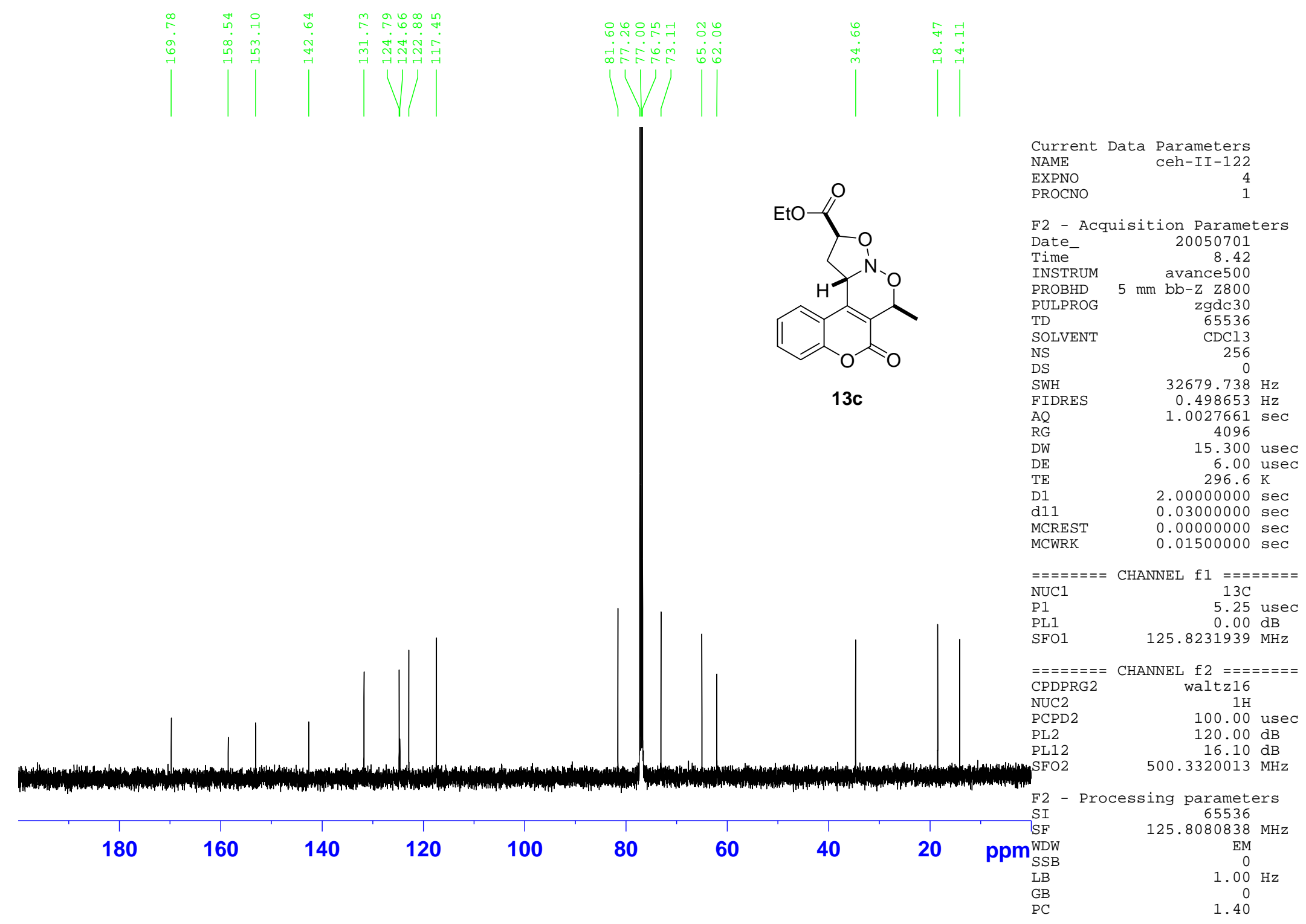




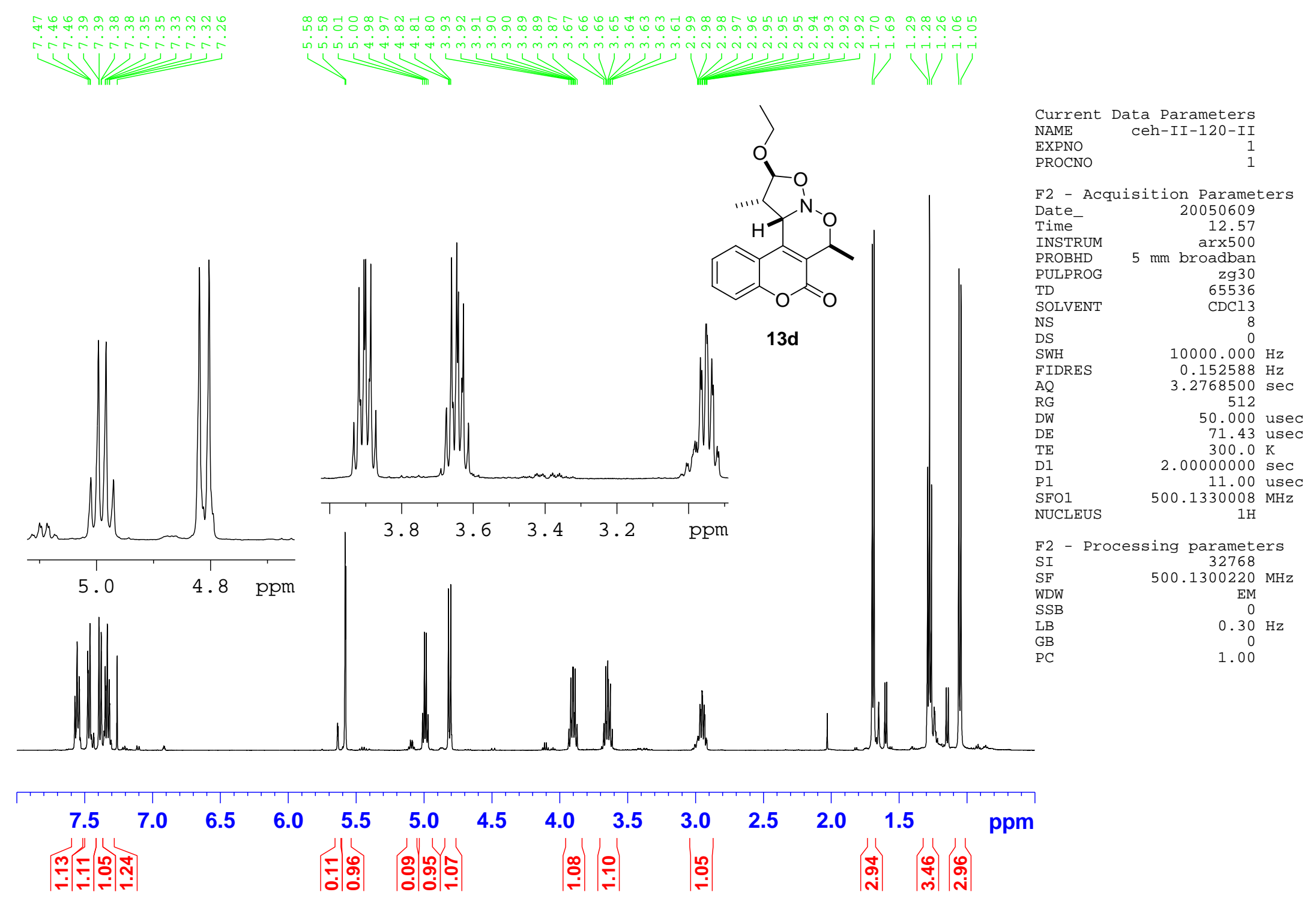




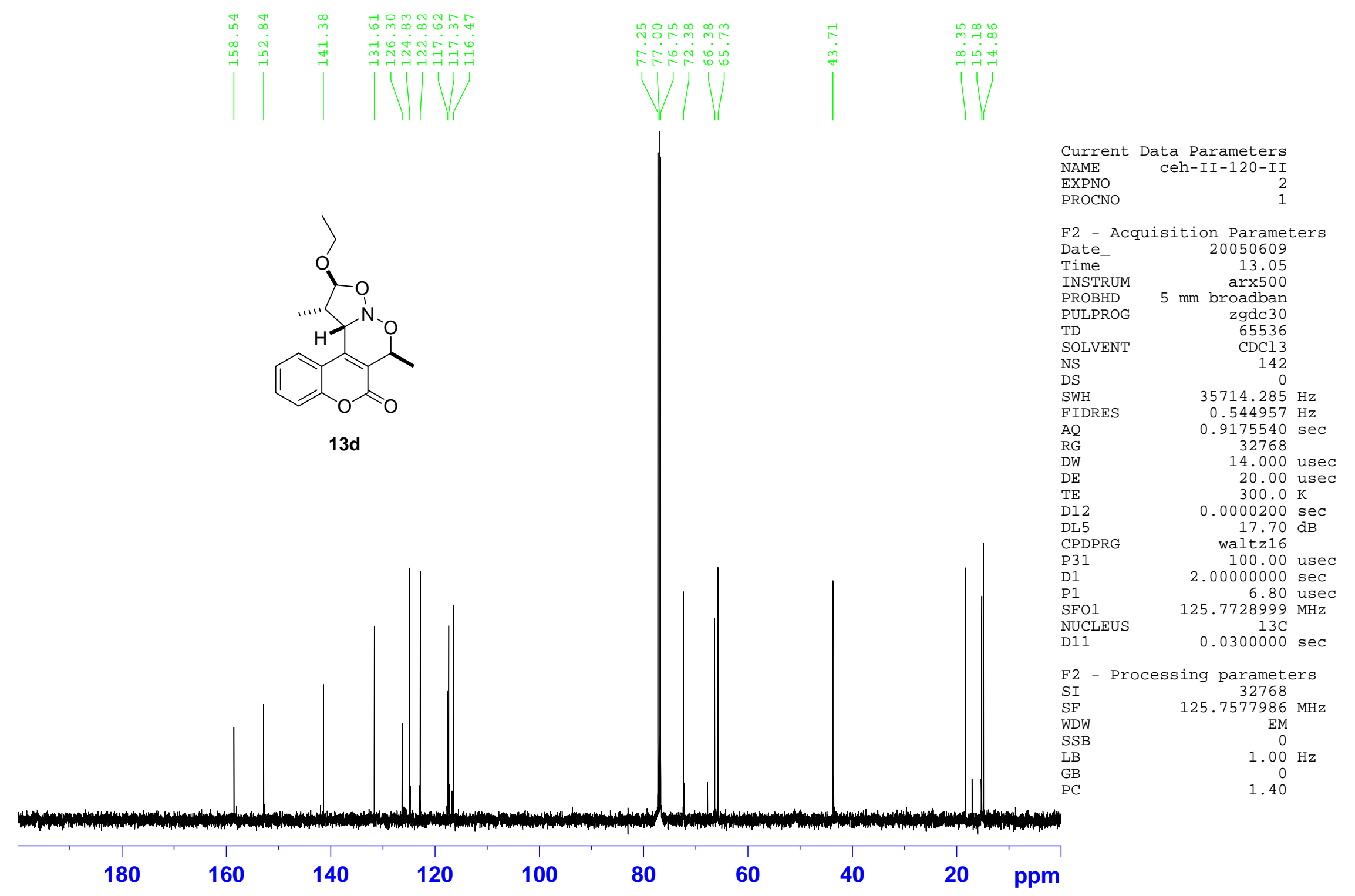




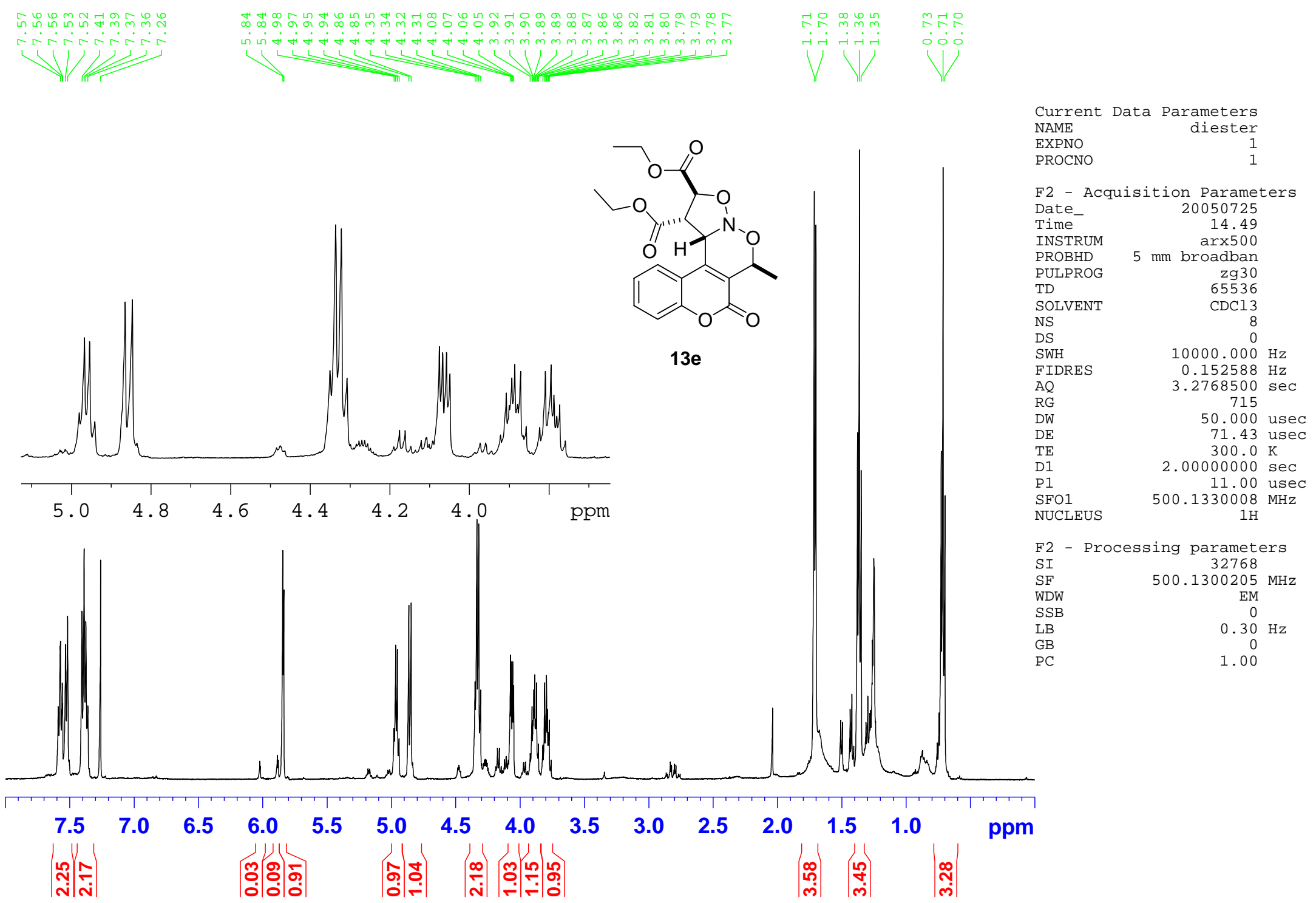




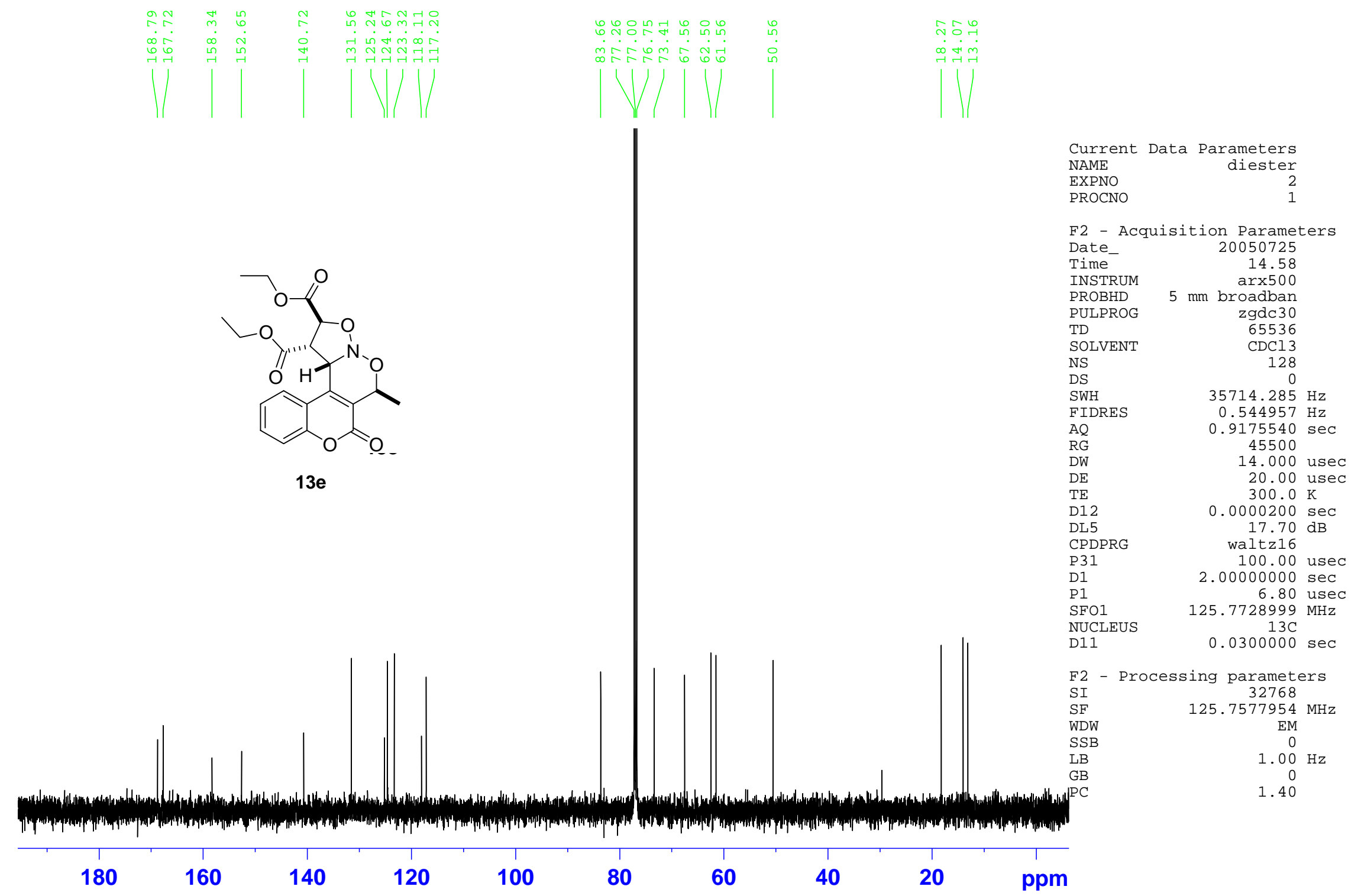

